\title{
Unmasking the Hidden Carbonyl Group Using Gold(I) \\ Catalysts and Alcohol Dehydrogenases: Design of a Thermodynamically-Driven Cascade Toward Optically Active Halohydrins
}

Sergio González-Granda, Lorena Escot, Iván Lavandera* and Vicente Gotor-Fernández*

Organic and Inorganic Chemistry Department. University of Oviedo. Avenida Julián Clavería 8, 33006 Oviedo, Spain. Phone number: +34985103452 (I.L.); +34985103454 (V.G.-F.); fax number: +34985103446.

E-mail: lavanderaivan@uniovi.es (I.L.); vicgotfer@uniovi.es (V.G.-F.).

Index (Page 1 out of 84)



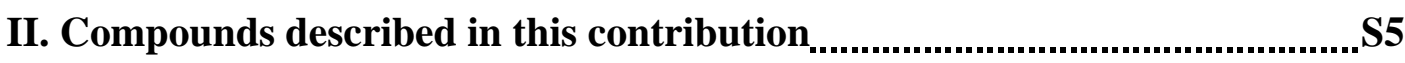

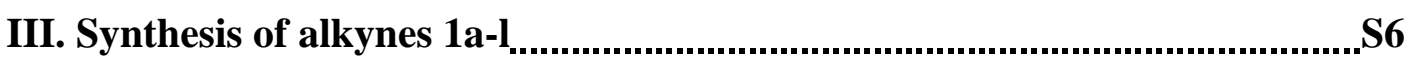

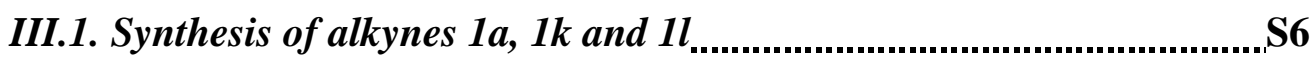

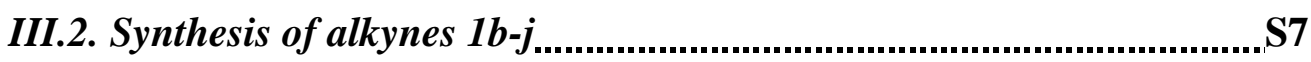

IV. Optimisation of gold(I)-catalysed hydration process of alkyne 1a.............S8

V. Full characterisation of $\alpha$-halomethyl ketones $2 \mathrm{a}-\mathrm{l}$, obtained through a gold(I)-catalysed hydration reaction

VI. Synthesis of halohydrins 3a-l as racemic standards for analytical measurement

VII. Screening of different ADHs in bioreduction experiments S15

VII.1. Bioreduction of a-chloromethyl ketone $2 a$ S15

VII.2. Bioreduction of a-chloromethyl ketone 2a using RasADH. S16

VII.3. Bioreduction of a-chloromethyl ketone 2a using LbADH S16

VII.4. Bioreduction of a-chloromethyl ketone 2 a using SyADH, TeSADH and ADH-T. S16 
VII.5. Bioreduction of a-chloromethyl ketone 2a using ADH-A. S17

VII.6. Bioreduction of a-chloromethyl ketone 2 a using commercial ADHs from Codexis.

VII.7. Screening of different ADHs for the asymmetric bioreduction of the ketone $2 j$.

VIII. Optimisation of the one-pot hydration-bioreduction cascade starting from alkyne 1a using $\mathrm{Lb} \mathrm{ADH}$.

IX. Study of the one-pot hydration-bioreduction cascade for 2a:

substrate concentration influence.

$X$. Scope of the one-pot cascade process and full characterisation of alkynes 3a-j.

X.I. Experimental protocol for the one-pot cascade reaction employing $E$. coli/LbADH to obtain enantioenriched alcohols $3 a-l$

X.II. Experimental protocol for the one-pot cascade reaction employing E. coli/ADH-A to obtain enantioenriched alcohols $3 a-k$

X.III. Experimental protocol for the one-pot cascade reaction employing Codexis KREDs to obtain alcohol $3 j$

$X . I V$. Experimental protocol for the one-pot cascade reaction employing E. coli/ADH-T to obtain alcohol $3 k$

XI. Scale-up of the one-pot cascade hydration-bioreduction process of alkyne 1a.

XII. Chemical epoxide formation from 2-chloro-1-phenylethan-1-ol (3a)

XIII. Development of a hydration-bioreduction-epoxide formation sequence to obtain both optically active epoxide 4a enantiomers

XIV. Analytical data

XIV.1. GC analyses for the determination of product percentages

XIV.2. GC calibration curves

XIV.3. GC analyses for the determination of ee values of alcohols $3 g, h, k$.

XIV.4. HPLC analyses for the determination of enantiomeric excess values of alcohols $3 a-f, i, j, l$ and epoxide $4 a$.

XV. Optical rotation values of halohydrins 3a-l obtained through the concurrent cascade approach

XVI. Reference section

XVII. NMR spectra 


\section{General considerations}

Chemical reagents and nicotinamide cofactors $\left(\mathrm{NADP}^{+}\right.$and $\left.\mathrm{NAD}^{+}\right)$were purchased from Sigma-Aldrich. The following gold(I) catalysts were also obtained from Sigma-Aldrich:

- $\quad$ [1,3-Bis(2,6-diisopropylphenyl)imidazol-2-ylidene]gold(I) [bis(trifluoromethanesulfonyl)imide] (IPrAuNTf 2 ),

- (Acetonitrile)[(2-biphenyl)di-tert-butylphosphine]gold(I) hexafluoroantimonate (JohnPhosAu(MeCN)SbF6) and

- [2-(Dicyclohexylphosphino)-3,6-dimethoxy-2',4',6'-triisopropyl-1,1'biphenyl]gold(I) bis(trifluoromethanesulfonyl)imide (BrettPhosAuNTf2),

while the syntheses of the other three gold complexes have been performed from its chloride derivatives:

- (Acetonitrile)[1,3-Bis(2,6-diisopropylphenyl)imidazol-2-ylidene]gold(I) hexafluoroantimonate (IPrAu(MeCN)SbF6),

- [(2-Biphenyl)di-tert-butylphosphine]gold(I) [bis(trifluoromethanesulfonyl)imide] (JohnPhosAuNTf 2 ) and

- $\quad$ (Tris(2,4-di-tert-butylphenyl)phosphite)gold(I) bis(trifluoromethanesulfonyl)imide $\left((\mathrm{ArO})_{3} \mathrm{PAuNTf}_{2}\right)$.

KREDs were received from Codexis Inc. Made in house ADHs were heterologously expressed in E. coli, these are Ralstonia species (RasADH), Sphingobium yanoikuyae (SyADH), Thermoanaerobacter species (ADH-T), Lactobacillus brevis (LbADH), Thermoanaerobacter ethanolicus (TeSADH) and Rhodococcus ruber (ADHA). Additional information about these alcohol dehydrogenases can be found in the main text of the manuscript and its reference section.

Non-commercially available acetylenes were chemically synthesised, exhibiting physical and spectral data in agreement with those reported in the literature (see below synthetic procedures and full characterisation). Cascade reactions were performed in a vial tube $[(2 \times 4) \mathrm{cm}]$.

NMR spectra were recorded on a MHz spectrometer including ${ }^{1} \mathrm{H},{ }^{13} \mathrm{C}$ and ${ }^{19} \mathrm{~F}$ NMR as well as bidimensional experiments. All chemical shifts $(\delta)$ are given in parts per million (ppm) and referenced to the residual solvent signal as internal standard.

Gas chromatography (GC) analyses were performed on an GC chromatograph equipped with a FID detector using a DB-1701 column (30 $\mathrm{m}$ x $0.23 \mathrm{~mm} \times 0.25 \mu \mathrm{m})$, HP- 
$1(30 \mathrm{~m} \times 0.32 \mathrm{~mm} \times 0.25 \mu \mathrm{m})$ o HP-5 $(30 \mathrm{~m} \times 0.32 \mathrm{~mm} \times 0.25 \mu \mathrm{m})$ for the determination of conversion values and a CP-Chirasil-DEX CB $(30 \mathrm{~m} \times 0.32 \mathrm{~mm} \times 0.25 \mu \mathrm{m})$ for the determination of enantiomeric excess values (see Section XIV).

High performance liquid chromatography (HPLC) analyses were performed on an HPLC chromatograph equipped with a VIS-UV detector using Chiralcel OJ-H column (25 $\mathrm{cm}$ x $4.6 \mathrm{~mm}, 5 \mu \mathrm{m}$ particle size), Chiralpak AD-H column $(25 \mathrm{~cm}$ x $4.6 \mathrm{~mm}, 5 \mu \mathrm{m}$ particle size) o Chiralpak IA column $(25 \mathrm{~cm} \times 4.6 \mathrm{~mm}, 5 \mu \mathrm{m}$ particle size $)$ for the measurement of enantiomeric excess values (see Section XIV).

Melting points were measured introducing the samples in open capillary tubes and the measurements are uncorrected. IR spectra were recorded in neat form, and $v_{\max }$ values are given in $\mathrm{cm}^{-1}$ for the main absorption bands. High resolution mass spectra (HRMS) experiments were carried out by electrospray ionisation in positive mode $\left(\mathrm{ESI}^{+}\right)$. Thinlayer chromatography (TLC) was conducted with Silica Gel 60 F254 precoated plates and visualised with a UV lamp, plus either potassium permanganate or vanillin stains. Column chromatographies were performed using silica gel 60 (230-240 mesh). 


\section{Compounds described in this contribution}

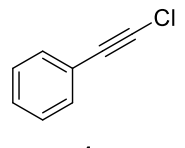

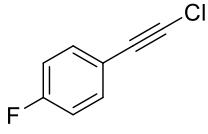

1b

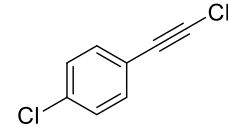

1c

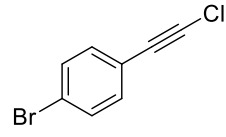

1d



$1 e$

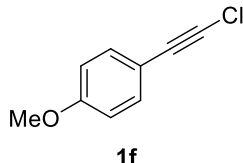<smiles>ClC#Cc1cccc(Cl)c1</smiles>

$1 \mathrm{~g}$<smiles>COc1cccc(C#CCl)c1</smiles>

$1 \mathrm{~h}$

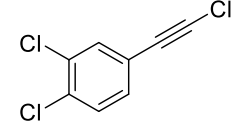

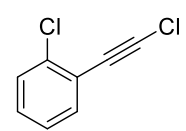

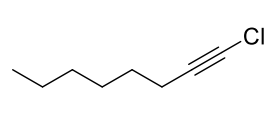

$1 \mathrm{k}$



11

Figure S1. Structure of haloalkynes 1a-l studied in this contribution.<smiles>O=C(CCl)c1ccccc1</smiles>

$2 a$<smiles>O=C(CCl)c1cccc(Cl)c1</smiles>

2g<smiles>O=C(CCl)c1ccc(F)cc1</smiles>

2b<smiles>O=C(Cl)c1ccc(Cl)cc1</smiles>

2c<smiles>O=C(Cl)c1ccc(Br)cc1</smiles>

2d<smiles>Cc1ccc(C(=O)CCl)cc1</smiles>

$2 e$<smiles>COc1ccc(C(=O)CCl)cc1</smiles>

$2 f$

Figure S2. Structure of prochiral $\alpha$-halomethyl ketones 2a-l studied in this contribution.<smiles>OC(CCl)c1ccccc1</smiles>

$3 a$<smiles>O=C(CCl)c1cccc(O[Na])c1</smiles>

2h<smiles>O=C(Cl)c1ccc(Cl)c(Cl)c1</smiles>

2i<smiles>O=C(CCl)c1ccccc1Cl</smiles>

2j<smiles>CCCCCCC(=O)CCl</smiles>

$2 \mathrm{k}$<smiles>O=C(Br)c1ccccc1</smiles>

21<smiles>COc1cccc(C(O)CCl)c1</smiles>

$3 g$<smiles>OC(CCl)c1ccc(F)cc1</smiles>

3b<smiles>OC(CCl)c1ccc(Cl)cc1</smiles>

$3 c$<smiles>OC(CCl)c1ccc(Br)cc1</smiles>

$3 d$<smiles>Cc1ccc(C(O)CCl)cc1</smiles>

$3 e$<smiles>COc1ccc(C(O)CCl)cc1</smiles>

$3 f$<smiles>OC(CCl)c1ccc(Cl)c(Cl)c1</smiles>

$3 i$<smiles>OC(CCl)c1ccccc1Cl</smiles>

3j<smiles>CCCCCCC(O)CCl</smiles>

3k<smiles>OC(CBr)c1ccccc1</smiles>

3!

Figure S3. Structure of $\alpha$-halohydrins 3a-l studied in this contribution.

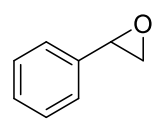

$4 a$

Figure S4. Structure of epoxide 4a studied in this contribution. 


\section{Synthesis of alkynes 1a-I}

\section{III.1. Synthesis of alkynes $1 a, 1 k$ and 11}

Compounds 1a and 1k were synthesised following adapted procedure of the one described by Nicolai et al. ${ }^{1}$



Scheme S1. Synthesis of chlorinated alkynes 1a and 1k.

$N$-chlorosuccinimide (NCS, $3.2 \mathrm{~g}, 24 \mathrm{mmol}, 1.20$ equiv) and silver acetate (AgOAc, 333.9 $\mathrm{mg}, 2 \mathrm{mmol}, 0.10$ equiv) were added in this order to a solution of the corresponding acetylene (20 mmol, 1.0 equiv) in acetone $(80 \mathrm{~mL})$, and the solution was refluxed overnight. After this time, the mixture was poured into ice, and the resulting aqueous layer extracted with pentane $(3 \times 20 \mathrm{~mL})$. The combined organic layers were washed with brine $(1 \times 25 \mathrm{~mL})$, dried over $\mathrm{Na}_{2} \mathrm{SO}_{4}$, filtered, and the solvent evaporated under reduced pressure. Purification by column chromatography $\left(\mathrm{SiO}_{2}\right.$, pentane) afforded the corresponding chlorinated alkyne 1a or 1k as smelly colorless oils (96\% of isolated yield 1a and $89 \%$ of isolated yield $\mathbf{1 k})$. The spectroscopic data of compounds $\mathbf{1 a}$ and $\mathbf{1 k}$ matched with the ones already reported in literature. ${ }^{1}$

Compound 11 was synthesised following an adapted procedure to the one described by Nicolai et al. ${ }^{1}$

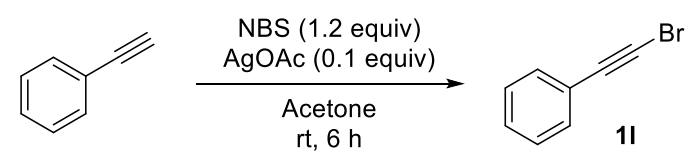

Scheme S2. Synthesis of brominated alkyne 11.

$\mathrm{N}$-bromosuccinimide (NBS, $2.13 \mathrm{~g}, 12 \mathrm{mmol}, 1.20$ equiv) and $\mathrm{AgNO}_{3}(170 \mathrm{mg}, 1 \mathrm{mmol}$, 0.10 equiv) were added in this order to a solution of phenylacetylene $(1.1 \mathrm{~mL}, 10 \mathrm{mmol}$, 1.0 equiv) in acetone $(60 \mathrm{~mL})$, and the mixture was stirred for $6 \mathrm{~h}$ at room temperature (rt). After complete consumption of the starting material (TLC analysis in hexane), the mixture was poured into ice-water and extracted with pentane $(3 \times 15 \mathrm{~mL})$. The combined 
organic extracts were dried over $\mathrm{Na}_{2} \mathrm{SO}_{4}$, filtered, and the solvent removed by evaporation under reduced pressure. The reaction crude was purified by column chromatography on silica gel, obtaining the brominated alkyne $\mathbf{1 l}$ as a yellowish oil (93\% isolated yield). The spectroscopic data of compound $\mathbf{1 1}$ matched with the ones already reported in literature. ${ }^{1}$

\section{III.2. Synthesis of alkynes $1 b-j$}

Alkynes 1b-j were synthesised through the Corey-Fuchs reaction following an adapted protocol to the one described by McIntosh et al. (Scheme S3). ${ }^{2}$
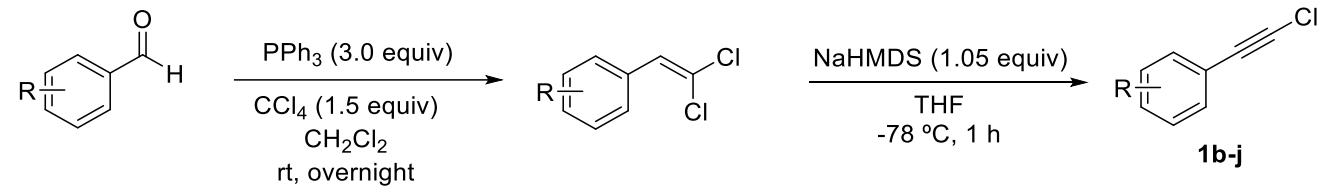

Scheme S3. Corey-Fuchs reaction to obtain chlorinated alkynes $\mathbf{1 b}-\mathbf{j}$.

$\mathrm{CCl}_{4}$ (3 mL, 31 mmol, 1.5 equiv) and $\mathrm{PPh}_{3}$ (15.8 g, $60 \mathrm{mmol}, 3$ equiv) were added at $\mathrm{rt}$ to a stirred solution of the corresponding benzaldehyde (20 mmol) in $\mathrm{CH}_{2} \mathrm{Cl}_{2}(170 \mathrm{~mL})$. After 16 hours, the black solution was concentrated in the rotary evaporator until around $50 \mathrm{~mL}$ of mixture remained. The residue was then purified via flash chromatography on silica gel, eluting with hexane to obtain the desired (2,2-dichlorovinyl)arenes as impure yellow oils.

Then, a $1 \mathrm{M}$ solution of NaHMDS in THF (10.05 mL, $10.05 \mathrm{mmol}, 1.05$ equiv) was added dropwise at $-78{ }^{\circ} \mathrm{C}$ to a stirred solution of the corresponding impure (2,2dichlorovinyl)arene $(10 \mathrm{mmol})$ in THF $(25 \mathrm{~mL})$. After 1 hour, the reaction was warmed to $0{ }^{\circ} \mathrm{C}$, quenched with a saturated $\mathrm{NH}_{4} \mathrm{Cl}$ aqueous solution $(20 \mathrm{~mL})$ and diluted with $\mathrm{H}_{2} \mathrm{O}$ (25 mL). Then, the solution was extracted with $\mathrm{Et}_{2} \mathrm{O}(3 \times 20 \mathrm{~mL})$, washed with brine (2 x $20 \mathrm{~mL}$ ), and the combined organic extracts dried over $\mathrm{Na}_{2} \mathrm{SO}_{4}$, filtered, and the solvent removed by evaporation under reduced pressure. Compounds $\mathbf{1 b}-\mathbf{j}$ were isolated by column chromatography over silica gel, eluting with hexane to obtain the desired chlorinated alkynes $\mathbf{1 b}-\mathbf{j}$ (81-93\% isolated yield). The spectroscopic data of compound 
$\mathbf{1 b}-\mathbf{h}$ and $\mathbf{1 j}$ matched with the ones already reported in literature $\left(\mathbf{1 b},{ }^{3} \mathbf{1 c},{ }^{3} \mathbf{1 d},{ }^{4} \mathbf{1 e},{ }^{4} \mathbf{1 f},{ }^{4}\right.$ $\mathbf{1 g},{ }^{5} \mathbf{1 h},{ }^{4}$ and $\left.\mathbf{1} \mathbf{j}^{6}\right)$, while for:

1,2-Dichloro-4-(chloroethynyl)benzene (1i): Colorless oil (86\% yield). $R_{f}$ (Hexane): 0.82. Mp: 41-42 ${ }^{\circ} \mathrm{C}$. IR: v 2227 and $811 \mathrm{~cm}^{-1} .{ }^{1} \mathrm{H}-\mathrm{NMR}\left(300.13 \mathrm{MHz}, \mathrm{CDCl}_{3}\right): \delta 7.52(d$, $J=1.9 \mathrm{~Hz}, 1 \mathrm{H}), 7.38(d, J=8.3 \mathrm{~Hz}, 1 \mathrm{H}), 7.25(d d, J=8.3,1.8 \mathrm{~Hz}, 1 \mathrm{H}) .{ }^{13} \mathrm{C}-\mathrm{NMR}(75.5$ $\left.\mathrm{MHz}, \mathrm{CDCl}_{3}\right): \delta 134.0(\mathrm{CH}), 133.6(\mathrm{C}), 133.0(\mathrm{C}), 131.5(\mathrm{CH}), 130.8(\mathrm{CH}), 122.4(\mathrm{C})$, $70.8(\mathrm{C}), 67.6(\mathrm{C})$. HRMS $\left(\mathrm{ESI}^{+}, \mathrm{m} / \mathrm{z}\right)$ : calcd for $\left(\mathrm{C}_{8} \mathrm{H}_{4} \mathrm{Cl}_{3}\right)^{+}(\mathrm{M}+\mathrm{H})^{+}:$204.9373; found 204.9367.

\section{Optimisation of gold(I)-catalysed hydration process of alkyne 1a}

Table S1. Preliminary study of the hydration process of chloroethynylbenzene (1a) using $\operatorname{IPrAuNTf}_{2}(5 \mathrm{~mol} \%)$ as catalyst at $40{ }^{\circ} \mathrm{C}$.

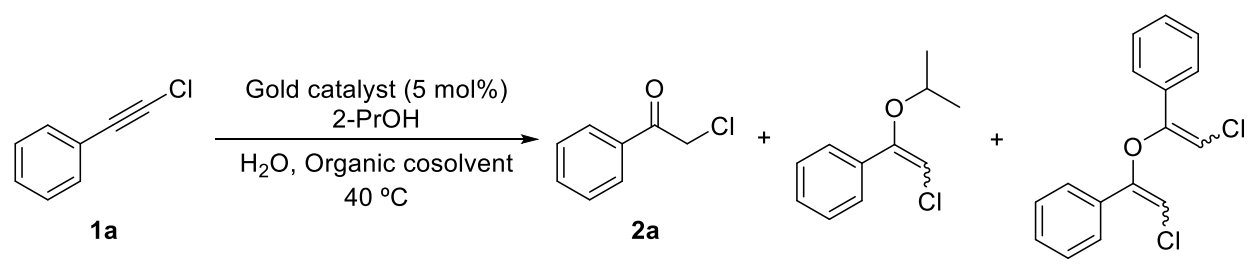

\begin{tabular}{cccccc}
\hline Entry & Reaction Medium $(v / v)$ & $\mathrm{t}(\mathrm{h})$ & $\begin{array}{c}\text { 1a } \\
(\%)^{\mathrm{a}}\end{array}$ & $\begin{array}{c}\mathbf{2 a} \\
(\%)^{\mathrm{a}}\end{array}$ & $\begin{array}{c}\text { By-products } \\
(\%)^{\mathrm{a}}\end{array}$ \\
\hline 1 & $4: 1: 1\left(\mathrm{H}_{2} \mathrm{O}: \mathrm{MeCN}: 2-\mathrm{PrOH}\right)$ & 4 & 29 & 25 & 45 \\
2 & $4: 1: 1\left(\mathrm{H}_{2} \mathrm{O}: \mathrm{MeCN}: 2-\mathrm{PrOH}\right)$ & 16 & 1 & 35 & 64 \\
3 & $4: 1\left(\mathrm{H}_{2} \mathrm{O}: 2-\mathrm{PrOH}\right)$ & 16 & $<1$ & 15 & 85 \\
4 & $4: 1\left(\mathrm{H}_{2} \mathrm{O}: \mathrm{MeCN}\right)+2$ equiv 2-PrOH & 16 & 1 & 82 & 17 \\
\hline
\end{tabular}

a Product percentages were determined by GC analysis using calibration curves (see Section XIV).

2-Chloro-1-phenylethan-1-one (2a): Colorless solid. $R_{\mathrm{f}}(10 \%$ EtOAc/hexane): 0.38. Mp: 55-56 ${ }^{\circ}$ C. IR: v 1679, 1596, 1581, 1170, 1161 and $751 \mathrm{~cm}^{-1} .{ }^{1} \mathrm{H}-\mathrm{NMR}$ (300.13 MHz, $\left.\mathrm{CDCl}_{3}\right): \delta 7.95(d d, J=8.5,1.4 \mathrm{~Hz}, 2 \mathrm{H}), 7.62(m, 1 \mathrm{H}), 7.50(d d t, J=8.3,6.7,1.1 \mathrm{~Hz}$, 2H), 4.73 ( $s, 2 \mathrm{H}) .{ }^{13} \mathrm{C}-\mathrm{NMR}\left(75.5 \mathrm{MHz}, \mathrm{CDCl}_{3}\right): \delta 191.5$ (C), $134.6(\mathrm{C}), 134.5(\mathrm{C}), 129.3$ $(2 \mathrm{CH}), 128.9(2 \mathrm{CH}), 45.6\left(\mathrm{CH}_{2}\right) .\left(\mathrm{ESI}^{+}, \mathrm{m} / \mathrm{z}\right)$ : calcd for $\left(\mathrm{C}_{8} \mathrm{H}_{8} \mathrm{ClO}\right)^{+}(\mathrm{M}+\mathrm{H})^{+}: 155.0258$; found 155.0260 . 
$(\boldsymbol{E}+\boldsymbol{Z})$-(2-Chloro-1-isopropoxyvinyl)benzene: Yellowish oil. $R_{\mathrm{f}}(10 \%$ EtOAc/hexane): 0.67. IR: $v$ 1704, 1283, 932 and $826 \mathrm{~cm}^{-1}$. Unfortunately, it has not been possible to separate the $Z$ and $E$ isomers, therefore the NMR data attached is a $(3: 1, E: Z)$ mixture of them: ${ }^{1} \mathrm{H}-\mathrm{NMR}\left(300.13 \mathrm{MHz}, \mathrm{CDCl}_{3}\right)$ : $\delta$ 7.44-7.35 (m, 6H), 7.24-7.11 $(m, 2 \mathrm{H}), 5.84(s$, $\left.1 \mathrm{H}, \mathrm{H}_{\mathrm{E}}\right), 5.81(s, 0.3 \mathrm{H}, \mathrm{Hz}), 4.13$ (hept, $\left.J=6.1 \mathrm{~Hz}, 1.3 \mathrm{H}, \mathrm{H}_{\mathrm{E}}+\mathrm{Hz}\right), 1.27(d, J=6.1 \mathrm{~Hz}$, 8H, $\mathrm{HE}+\mathrm{Hz}) .{ }^{13} \mathrm{C}-\mathrm{NMR}\left(75.5 \mathrm{MHz}, \mathrm{CDCl}_{3}\right): \delta 154.9(\mathrm{C}), 139.9(\mathrm{C}), 135.0(\mathrm{CH}), 134.5$ $(2 \mathrm{CH}), 129.4(\mathrm{CH}), 129.0(2 \mathrm{CH}), 128.7,126.9(2 \mathrm{CH}), 103.4(\mathrm{CH}), 72.9(\mathrm{CH}), 22.6$ $\left(2 \mathrm{CH}_{3}\right) .\left(\mathrm{ESI}^{+}, \mathrm{m} / \mathrm{z}\right)$ : calcd for $\left(\mathrm{C}_{11} \mathrm{H}_{14} \mathrm{ClO}\right)^{+}(\mathrm{M}+\mathrm{H})^{+}$: 197.0728; found 197.0724.

Table S2. Screening of gold(I) catalysts $(5 \mathrm{~mol} \%)$ in the hydration reaction of chloroethynylbenzene (1a) using a $\mathrm{H}_{2} \mathrm{O}: \mathrm{MeCN}$ mixture (4:1 v/v) as solvent and 2 equivalents of 2-PrOH at $40{ }^{\circ} \mathrm{C}$ for $16 \mathrm{~h}$.

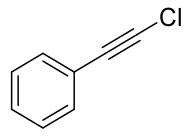

$1 \mathrm{a}$ $(100 \mathrm{mM})$

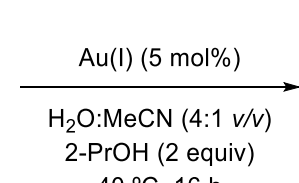
$40^{\circ} \mathrm{C}, 16 \mathrm{~h}$

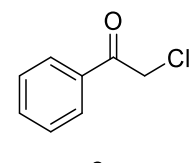

$2 a$

\begin{tabular}{ccccc}
\hline Entry & Catalyst & $\mathbf{1 a}(\%)^{\mathrm{a}}$ & $\mathbf{2 a}(\%)^{\mathrm{a}}$ & ${\text { By-products }(\%)^{\mathrm{a}}}^{\mathrm{a}}$ \\
\hline 1 & IPrAuNTf $_{2}$ & 1 & 82 & 17 \\
2 & $\mathrm{IPrAu}(\mathrm{MeCN}) \mathrm{SbF}_{6}$ & 85 & 11 & 3 \\
\hline 3 & ${\text { JohnPhosAu}(\mathrm{MeCN})_{\mathrm{SbF}}}_{6}$ & 48 & 41 & 11 \\
4 & JohnPhosAuNTf $_{2}$ & $<1$ & 81 & 19 \\
\hline 5 & BrettPhosAuNTf $_{2}$ & $<1$ & 85 & 15 \\
\hline 6 & $(\mathrm{ArO})_{3}$ PAuNTf $_{2}$ & 45 & 40 & 14 \\
\hline
\end{tabular}

${ }^{\text {a }}$ Product percentages were determined by GC analysis using calibration curves. 
Table S3. Screening of reaction medium, temperature and equivalents of 2-PrOH in the hydration process of chloroethynylbenzene (1a) using $\operatorname{IPrAuNTf}_{2}(5 \mathrm{~mol} \%)$.

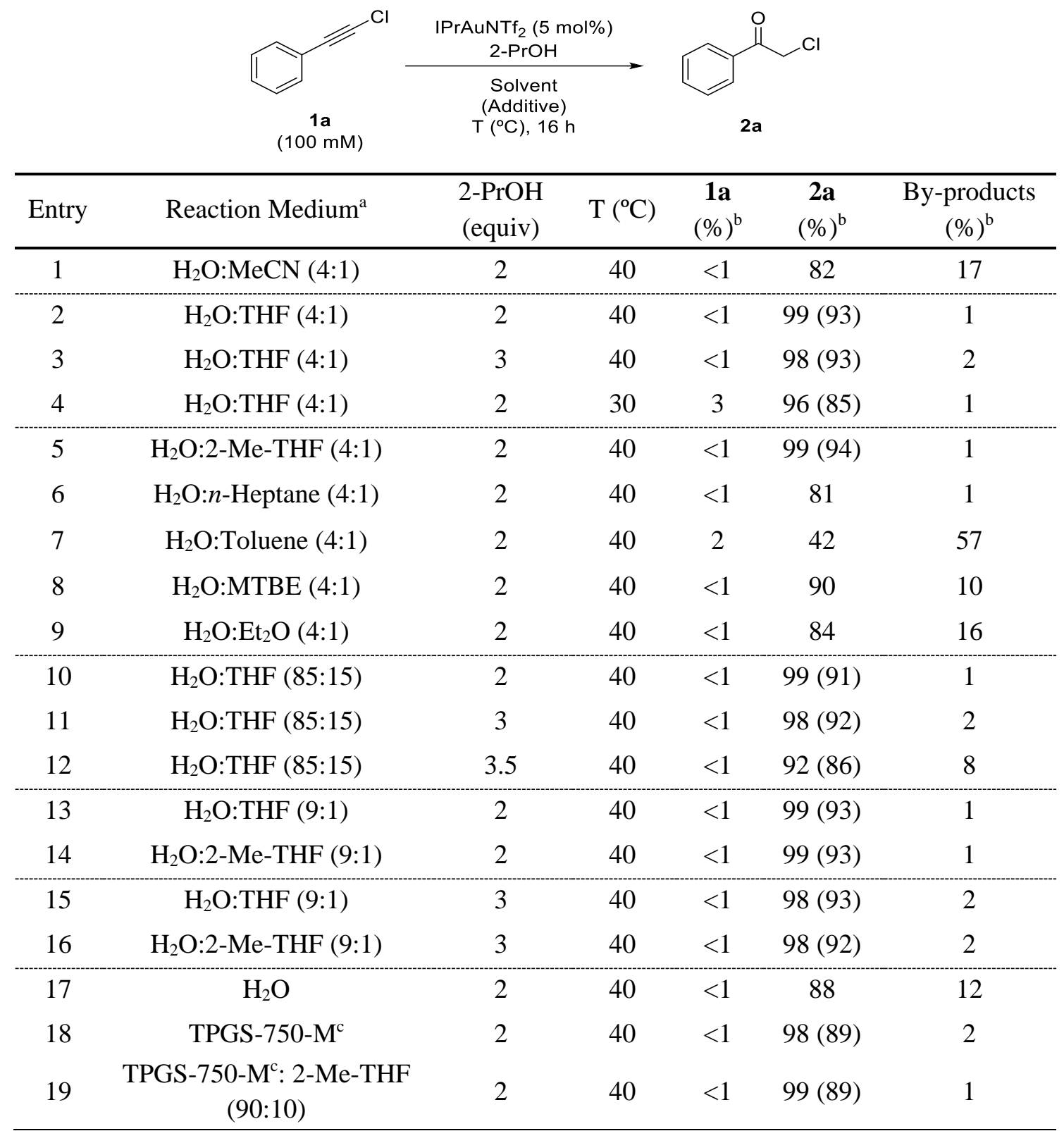

\footnotetext{
${ }^{a}$ Volume ratios appear in parentheses.

${ }^{\mathrm{b}}$ Product percentages were determined by GC analysis using calibration curves. Isolated yields of $\alpha$ chloromethyl ketone 2a in parentheses.

${ }^{\mathrm{c}}$ This is a commercially available water solution that includes the surfactant in $2 \% \mathrm{w} / \mathrm{v}$.
} 
V. Full characterisation of $\alpha$-halomethyl ketones 2 a-l, obtained through a gold(I)catalysed hydration reaction

2-Chloro-1-phenylethan-1-one (2a): Colorless solid (91\%). See Section IV for full compound characterisation.

2-Chloro-1-(4-fluorophenyl)ethan-1-one (2b): Colorless solid (92\%). $R_{\mathrm{f}} \quad(10 \%$ EtOAc/hexane): 0.49. Mp: 49-50 ${ }^{\circ} \mathrm{C}$. IR: $v$ 1692, 1596, 1581, 1210, 1177 and $795 \mathrm{~cm}^{-1}$. ${ }^{1} \mathrm{H}-\mathrm{NMR}\left(300.13 \mathrm{MHz}, \mathrm{CDCl}_{3}\right): \delta 7.99(m, 2 \mathrm{H}), 7.17(d d, J=9.6,7.6 \mathrm{~Hz}, 2 \mathrm{H}), 4.67(s$, 2H). ${ }^{13} \mathrm{C}-\mathrm{NMR}\left(75.5 \mathrm{MHz}, \mathrm{CDCl}_{3}\right): \delta 189.7(\mathrm{C}), 166.2(d, J=256.7, \mathrm{C}), 131.4(d, J=9.8$ $\mathrm{Hz}, 2 \mathrm{CH}), 130.6(\mathrm{C}), 116.2(d, J=21.9 \mathrm{~Hz}, 2 \mathrm{CH}), 46.6\left(\mathrm{CH}_{2}\right) .{ }^{19} \mathrm{~F}-\mathrm{RMN}(282 \mathrm{MHz}$, $\left.\mathrm{CDCl}_{3}\right): \delta-103.05$. $\left(\mathrm{ESI}^{+}, \mathrm{m} / \mathrm{z}\right)$ : calcd for $\left(\mathrm{C}_{8} \mathrm{H}_{6} \mathrm{ClFNaO}\right)^{+}(\mathrm{M}+\mathrm{Na})^{+}: 194.9983$; found 194.9988.

2-Chloro-1-(4-chlorophenyl)ethan-1-one (2c): Colorless solid $(91 \%) . \quad R_{\mathrm{f}} \quad(10 \%$ EtOAc/hexane): 0.35. Mp: 100-101 ${ }^{\circ} \mathrm{C}$. IR: v 1684, 1587, 1570, 1275, 1261 and 764 $\mathrm{cm}^{-1} .{ }^{1} \mathrm{H}-\mathrm{NMR}\left(300.13 \mathrm{MHz}, \mathrm{CDCl}_{3}\right): \delta 7.91(m, 2 \mathrm{H}), 7.48(\mathrm{~m}, 2 \mathrm{H}), 4.66(s, 2 \mathrm{H}) .{ }^{13} \mathrm{C}-$ NMR (75.5 MHz, $\left.\mathrm{CDCl}_{3}\right): \delta 190.1(\mathrm{C}), 140.6(\mathrm{C}), 133.0(\mathrm{C}), 130.0(2 \mathrm{CH}), 129.3(2 \mathrm{CH})$, $46.6\left(\mathrm{CH}_{2}\right) .\left(\mathrm{ESI}^{+}, \mathrm{m} / \mathrm{z}\right)$ : calcd for $\left(\mathrm{C}_{8} \mathrm{H}_{6} \mathrm{Cl}_{2} \mathrm{NaO}\right)^{+}(\mathrm{M}+\mathrm{Na})^{+}:$210.9688; found 210.9693 .

2-Chloro-1-(4-bromophenyl)ethan-1-one (2d): Colorless solid $(88 \%)$. $R_{\mathrm{f}} \quad(10 \%$ EtOAc/hexane): 0.39. Mp: 120-121 ${ }^{\circ} \mathrm{C}$. IR: $v$ 1683, 1583, 1569, 1392, 1268, 1260 and $768 \mathrm{~cm}^{-1} .{ }^{1} \mathrm{H}-\mathrm{NMR}\left(300.13 \mathrm{MHz}, \mathrm{CDCl}_{3}\right): \delta 7.83(m, 2 \mathrm{H}), 7.65(m, 2 \mathrm{H}), 4.66(s, 2 \mathrm{H})$. ${ }^{13} \mathrm{C}-\mathrm{NMR}\left(75.5 \mathrm{MHz}, \mathrm{CDCl}_{3}\right): \delta 190.3(\mathrm{C}), 132.9(\mathrm{C}), 132.3(2 \mathrm{CH}), 130.1(2 \mathrm{CH}), 129.4$ (C), $45.6\left(\mathrm{CH}_{2}\right)$. $\left(\mathrm{ESI}^{+}, \mathrm{m} / \mathrm{z}\right)$ : calcd for $\left(\mathrm{C}_{8} \mathrm{H}_{6} \mathrm{BrClNaO}\right)^{+}(\mathrm{M}+\mathrm{Na})^{+}$: 254.9183; found 254.9188

2-Chloro-1-(p-tolyl)ethan-1-one (2e): Colorless solid (93\%). $R_{\mathrm{f}}(20 \%$ EtOAc/hexane): 0.69. Mp: 58-59 ${ }^{\circ} \mathrm{C}$. IR: $v$ 1691, 1582, 1566, 1267, 1234 and $764 \mathrm{~cm}^{-1} .{ }^{1} \mathrm{H}-\mathrm{NMR}(300.13$ $\left.\mathrm{MHz}_{\mathrm{CDCl}}\right): \delta 7.86(m, 2 \mathrm{H}), 7.29(d t, J=8.1,0.9 \mathrm{~Hz}, 2 \mathrm{H}), 4.69(s, 2 \mathrm{H}), 2.42(s, 3 \mathrm{H})$. ${ }^{13} \mathrm{C}-\mathrm{NMR}\left(75.5 \mathrm{MHz}, \mathrm{CDCl}_{3}\right): \delta 190.7$ (C), 145.1 (C), 131.8 (C), $129.6(2 \mathrm{CH}), 128.6(\mathrm{C})$, $46.0\left(\mathrm{CH}_{2}\right), 21.8\left(\mathrm{CH}_{3}\right) .\left(\mathrm{ESI}^{+}, \mathrm{m} / \mathrm{z}\right)$ : calcd for $\left(\mathrm{C}_{9} \mathrm{H}_{10} \mathrm{ClO}\right)^{+}(\mathrm{M}+\mathrm{H})^{+}:$169.0415; found 169.0416 . 
2-Chloro-1-(4-methoxyphenyl)ethan-1-one (2f): Colorless solid (89\%). $R_{\mathrm{f}}(10 \%$ EtOAc/hexane): 0.32. Mp: 169-171 ${ }^{\circ} \mathrm{C}$. IR: v 1688, 1440, 1420, 1267, 1260 and 764 $\mathrm{cm}^{-1} .{ }^{1} \mathrm{H}-\mathrm{NMR}\left(300.13 \mathrm{MHz}, \mathrm{CDCl}_{3}\right): \delta 7.95(m, 2 \mathrm{H}), 6.97(m, 2 \mathrm{H}), 4.66(s, 2 \mathrm{H}), 3.89(s$, 3H). ${ }^{13} \mathrm{C}-\mathrm{NMR}\left(75.5 \mathrm{MHz}, \mathrm{CDCl}_{3}\right): \delta 189.7(\mathrm{C}), 164.2(\mathrm{C}), 130.9(2 \mathrm{CH}), 127.2(\mathrm{C}), 114.1$ (2CH), $55.6\left(\mathrm{CH}_{3}\right), 45.7\left(\mathrm{CH}_{2}\right)$. $\left(\mathrm{ESI}^{+}, \mathrm{m} / \mathrm{z}\right)$ : calcd for $\left(\mathrm{C}_{9} \mathrm{H}_{10} \mathrm{ClO}_{2}\right)^{+}(\mathrm{M}+\mathrm{Na})^{+}$: 185.0364; found 185.0366 .

2-Chloro-1-(3-chlorophenyl)ethan-1-one (2g): Colorless solid $(86 \%)$. $R_{\mathrm{f}} \quad(10 \%$ EtOAc/hexane): 0.37. Mp:93-95 ${ }^{\circ} \mathrm{C} .{ }^{1} \mathrm{H}-\mathrm{NMR}\left(300.13 \mathrm{MHz}, \mathrm{CDCl}_{3}\right): \delta 7.94\left(t_{\text {apparent }}, J=\right.$ $1.8 \mathrm{~Hz}, 1 \mathrm{H}), 7.84(m, 1 \mathrm{H}), 7.61(m, 1 \mathrm{H}), 7.46$ (tapparent, $J=7.9 \mathrm{~Hz}, 1 \mathrm{H}), 4.69(s, 2 \mathrm{H}) .{ }^{13} \mathrm{C}-$ NMR (75.5 MHz, $\left.\mathrm{CDCl}_{3}\right)$ : $\delta 190(\mathrm{C}), 135.7(\mathrm{C}), 135.3(\mathrm{C}), 133.9(\mathrm{CH}), 130.2(\mathrm{CH}), 128.6$ $(\mathrm{CH}), 126.6(\mathrm{CH}), 45.8\left(\mathrm{CH}_{2}\right)$.

2-Chloro-1-(3-methoxyphenyl)ethan-1-one (2h): Colorless solid (79\%). $R_{\mathrm{f}}(10 \%$ EtOAc/hexane): 0.34. Mp: 76-78 ${ }^{\circ} \mathrm{C}{ }^{1} \mathrm{H}-\mathrm{NMR}\left(300.13 \mathrm{MHz}, \mathrm{CDCl}_{3}\right): \delta 7.51(\mathrm{~m}, 2 \mathrm{H}), 7.42$ ( $\left.t_{\text {apparent }}, J=8.0 \mathrm{~Hz}, 1 \mathrm{H}\right), 7.16(d d d, J=8.2,2.6,0.9 \mathrm{~Hz}, 1 \mathrm{H}), 4.72(s, 2 \mathrm{H}), 3.87(s, 3 \mathrm{H})$. ${ }^{13} \mathrm{C}-\mathrm{NMR}\left(75.5 \mathrm{MHz}, \mathrm{CDCl}_{3}\right): \delta 190.9(\mathrm{C}), 160.0(\mathrm{C}), 135.5(\mathrm{C}), 239.9(\mathrm{CH}), 120.9(\mathrm{CH})$, $120.4(\mathrm{CH}), 112.8(\mathrm{CH}), 55.5\left(\mathrm{CH}_{3}\right), 46.1\left(\mathrm{CH}_{2}\right)$.

2-Chloro-1-(3,4-dichlorophenyl)ethan-1-one (2i): Colorless solid (84\%). $R_{\mathrm{f}}(20 \%$ EtOAc/hexane): 0.55. Mp: 45-46 ${ }^{\circ} \mathrm{C}$. IR: $v$ 1701, 1582, 1550, 1267, 1260 and $768 \mathrm{~cm}^{-1}$. ${ }^{1} \mathrm{H}-\mathrm{NMR}\left(300.13 \mathrm{MHz}, \mathrm{CDCl}_{3}\right): \delta 8.05(d, J=2.1 \mathrm{~Hz}, 1 \mathrm{H}), 7.79(d d, J=8.4,2.1 \mathrm{~Hz}$, $1 \mathrm{H}), 7.59(d, J=8.2 \mathrm{~Hz}, 1 \mathrm{H}), 4.64(s, 2 \mathrm{H}) .{ }^{13} \mathrm{C}-\mathrm{NMR}\left(75.5 \mathrm{MHz}, \mathrm{CDCl}_{3}\right): \delta 189.2(\mathrm{C})$, $138.8(\mathrm{C}), 133.8(\mathrm{C}), 133.6(\mathrm{C}), 131.1(\mathrm{CH}), 130.6(\mathrm{CH}), 127.6(\mathrm{CH}), 45.4\left(\mathrm{CH}_{2}\right) .\left(\mathrm{ESI}^{+}\right.$, $\mathrm{m} / \mathrm{z})$ : calcd for $\left(\mathrm{C}_{8} \mathrm{H}_{5} \mathrm{Cl}_{3} \mathrm{NaO}\right)^{+}(\mathrm{M}+\mathrm{Na})^{+}:$244.9298; found 244.9299 .

2-Chloro-1-(2-chlorophenyl)ethan-1-one (2j): Colorless solid $(91 \%) . \quad R_{\mathrm{f}} \quad(20 \%$ EtOAc/hexane): 0.67. Mp: 73-74 ${ }^{\circ} \mathrm{C}$. IR: $v$ 1680, 1587, 1577, 1271, 1255 and $769 \mathrm{~cm}^{-1}$. ${ }^{1} \mathrm{H}-\mathrm{NMR}\left(300.13 \mathrm{MHz}, \mathrm{CDCl}_{3}\right): \delta 7.56(m, 1 \mathrm{H}), 7.44(m, 2 \mathrm{H}), 7.37(m, 1 \mathrm{H}), 4.71(s, 2 \mathrm{H})$. ${ }^{13} \mathrm{C}-\mathrm{NMR}\left(75.5 \mathrm{MHz}, \mathrm{CDCl}_{3}\right): \delta 194.7(\mathrm{C}), 136.6(\mathrm{C}), 133.3(\mathrm{CH}), 131.7(\mathrm{C}), 131.1(\mathrm{CH})$, $130.4(\mathrm{CH}), 127.7(\mathrm{CH}), 49.2\left(\mathrm{CH}_{2}\right)$. $\left(\mathrm{ESI}^{+}, \mathrm{m} / \mathrm{z}\right)$ : calcd for $\left(\mathrm{C}_{8} \mathrm{H}_{16} \mathrm{ClO}\right)^{+}(\mathrm{M}+\mathrm{H})^{+}$: 163.0884; found 163.0887. 
1-Chlorooctan-2-one (2k): Yellowish oil (96\%). $R_{\mathrm{f}}(5 \%$ EtOAc/hexane): 0.60. IR: $v$ 1693 and $769 \mathrm{~cm}^{-1} .{ }^{1} \mathrm{H}-\mathrm{NMR}\left(300.13 \mathrm{MHz}, \mathrm{CDCl}_{3}\right): \delta 4.07(s, 2 \mathrm{H}), 2.58(t, J=7.4 \mathrm{~Hz}$, 2H), $1.61(m, 2 \mathrm{H}), 1.29(m, 6 \mathrm{H}), 0.88(m, 3 \mathrm{H}) .{ }^{13} \mathrm{C}-\mathrm{NMR}\left(75.5 \mathrm{MHz}, \mathrm{CDCl}_{3}\right): \delta 202.8$ (C), $48.2\left(\mathrm{CH}_{2}\right), 39.7\left(\mathrm{CH}_{2}\right), 31.5\left(\mathrm{CH}_{2}\right), 28.7\left(\mathrm{CH}_{2}\right), 23.6\left(\mathrm{CH}_{2}\right), 22.5\left(\mathrm{CH}_{2}\right), 14.0\left(\mathrm{CH}_{3}\right)$. $\left(\mathrm{ESI}^{+}, \mathrm{m} / \mathrm{z}\right)$ : calcd for $\left(\mathrm{C}_{8} \mathrm{H}_{16} \mathrm{ClO}\right)^{+}(\mathrm{M}+\mathrm{H})^{+}:$163.0889; found 163.0883 .

In the gold-catalysed synthesis of ketone $\mathbf{2 k}$ and additional product was identified, namely the dimer (Z)-1-chloro-2- $\{[(Z)-1$-chlorooct-1-en-2-yl]oxy $\}$ oct-1-ene, which was obtained in $3 \%$ isolated yield after column chromatography on silica gel.

(Z)-1-Chloro-2- $\{[(Z)-1-c h l o r o o c t-1-e n-2-y l]$ oxy $\}$ oct-1-ene: Yellowish oil. $R_{\mathrm{f}} \quad(n$ hexane): 0.46. ${ }^{1} \mathrm{H}-\mathrm{NMR}\left(300.13 \mathrm{MHz}, \mathrm{CDCl}_{3}\right): \delta 5.44(s, 2 \mathrm{H}), 2.12(t, J=7.5 \mathrm{~Hz}, 4 \mathrm{H})$, 1.46-1.56 (m, 4H), $1.30(m, 12 \mathrm{H}), 0.89(m, 6 \mathrm{H}) .{ }^{13} \mathrm{C}-\mathrm{NMR}\left(75.5 \mathrm{MHz}, \mathrm{CDCl}_{3}\right): \delta 153.2$ (2C), $100.1(2 \mathrm{CH}), 32.3\left(2 \mathrm{CH}_{2}\right), 31.5\left(2 \mathrm{CH}_{2}\right), 28.7\left(2 \mathrm{CH}_{2}\right), 26.6\left(2 \mathrm{CH}_{2}\right), 22.5\left(2 \mathrm{CH}_{2}\right)$, 14.1 $\left(2 \mathrm{CH}_{3}\right)$. $\left(\mathrm{APCI}^{+}, \mathrm{m} / \mathrm{z}\right)$ : calcd for $\left(\mathrm{C}_{16} \mathrm{H}_{29} \mathrm{Cl}_{2} \mathrm{O}\right)^{+}(\mathrm{M}+\mathrm{H})^{+}:$307.1590; found 307.1587.

2-Bromo-1-phenylethan-1-one (2I): Colorless solid (91\%). $R_{\mathrm{f}}(20 \%$ EtOAc/hexane): 0.63. Mp: 51-52 ${ }^{\circ} \mathrm{C}$. IR: $v$ 1701, 1583, 1560, 1267, 1260 and $762 \mathrm{~cm}^{-1}$. ${ }^{1} \mathrm{H}-\mathrm{NMR}(300.13$ $\left.\mathrm{MHz}, \mathrm{CDCl}_{3}\right): \delta 8.00(m, 2 \mathrm{H}), 7.62(m, 1 \mathrm{H}), 7.50(d d t, J=8.2,6.7,1.1 \mathrm{~Hz}, 2 \mathrm{H}), 4.46(s$, 2H). ${ }^{13} \mathrm{C}-\mathrm{NMR}\left(75.5 \mathrm{MHz}, \mathrm{CDCl}_{3}\right): \delta 191.3(\mathrm{C}), 134.0(2 \mathrm{C}), 129.0(2 \mathrm{CH}), 128.9(2 \mathrm{CH})$, $30.9\left(\mathrm{CH}_{2}\right) .\left(\mathrm{ESI}^{+}, \mathrm{m} / \mathrm{z}\right)$ : calcd for $\left(\mathrm{C}_{8} \mathrm{H}_{8} \mathrm{BrO}\right)^{+}(\mathrm{M}+\mathrm{H})^{+}$: 198.9753 ; found 198.9753 .

\section{Synthesis of halohydrins 3a-l as racemic standards for analytical measurement}

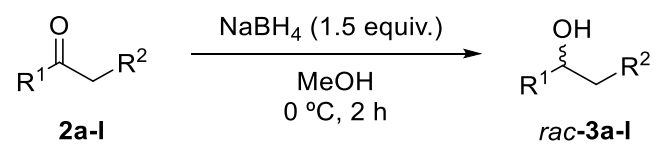

Scheme S4. Synthesis of racemic halohydrins 3a-1.

Sodium borohydride $\left(\mathrm{NaBH}_{4}, 2.9 \mathrm{mg}, 0.075 \mathrm{mmol}\right)$ was added in portions, at $0{ }^{\circ} \mathrm{C}$, to a stirred solution of the corresponding $\alpha$-halomethyl ketone $\mathbf{2 a - 1}(0.05 \mathrm{mmol}, 50 \mathrm{mM})$ in dry $\mathrm{MeOH}(1 \mathrm{~mL})$. The reaction was slowly warmed to room temperature and stirred for additional 2 hours. Then, a $\mathrm{NH}_{4} \mathrm{Cl}$ aqueous solution $(2 \mathrm{~mL})$ was added, and the mixture extracted with EtOAc ( $3 \times 1 \mathrm{~mL})$. The organic phases were combined and washed with 
brine ( $3 \mathrm{~mL}$ ), dried over $\mathrm{Na}_{2} \mathrm{SO}_{4}$, filtered and concentrated under reduced pressure. The corresponding reaction crudes were purified by column chromatography on silica gel, obtaining the halohydrins 3a-I with excellent purity (49-97\% isolated yield, Table S4).

Table S4. Chemical reduction of ketones $\mathbf{2 a - I}$ to obtain racemic alcohols 3a-l.

\begin{tabular}{|c|c|c|c|c|}
\hline Entry & Ketone 2a-l & $\mathrm{R}^{1}$ & $\mathrm{R}^{2}$ & Isolated yield 3a-l (\%) \\
\hline 1 & $2 \mathbf{a}$ & $\mathrm{Ph}$ & $\mathrm{Cl}$ & 93 \\
\hline 2 & $2 \mathbf{b}$ & $4-\mathrm{F}-\mathrm{C}_{6} \mathrm{H}_{4}$ & $\mathrm{Cl}$ & 95 \\
\hline 3 & $2 c$ & 4- $\mathrm{Cl}-\mathrm{C}_{6} \mathrm{H}_{4}$ & $\mathrm{Cl}$ & 87 \\
\hline 4 & 2d & $4-\mathrm{Br}-\mathrm{C}_{6} \mathrm{H}_{4}$ & $\mathrm{Cl}$ & 85 \\
\hline 5 & $2 e$ & $4-\mathrm{Me}-\mathrm{C}_{6} \mathrm{H}_{4}$ & $\mathrm{Cl}$ & 94 \\
\hline 6 & $2 f$ & $4-\mathrm{OMe}-\mathrm{C}_{6} \mathrm{H}_{4}$ & $\mathrm{Cl}$ & 97 \\
\hline 7 & $2 \mathrm{~g}$ & 4-Cl- $\mathrm{C}_{6} \mathrm{H}_{4}$ & $\mathrm{Cl}$ & 49 \\
\hline 8 & $2 h$ & $3-\mathrm{OMe}-\mathrm{C}_{6} \mathrm{H}_{4}$ & $\mathrm{Cl}$ & 78 \\
\hline 9 & $2 \mathbf{i}$ & $3,4-\mathrm{Cl}_{2}-\mathrm{C}_{6} \mathrm{H}_{3}$ & $\mathrm{Cl}$ & 89 \\
\hline 10 & $2 \mathbf{j}$ & $2-\mathrm{Cl}-\mathrm{C}_{6} \mathrm{H}_{4}$ & $\mathrm{Cl}$ & 84 \\
\hline 11 & $2 \mathbf{k}$ & $\mathrm{C}_{6} \mathrm{H}_{13}$ & $\mathrm{Cl}$ & 88 \\
\hline 12 & 21 & $\mathrm{Ph}$ & $\mathrm{Br}$ & $76^{\mathrm{b}}$ \\
\hline
\end{tabular}

\footnotetext{
${ }^{a}$ Isolated yields of alcohols 3a-l after column chromatography.

${ }^{b}$ The formation of styrene oxide was also observed and quantified ( $22 \%$ isolated yield).
} 


\section{Screening of different ADHs in bioreduction experiments}

\section{VII.1. Bioreduction of $\alpha$-chloromethyl ketone $2 a$}

Table S5. Screening of different ADHs for the asymmetric bioreduction of $\mathbf{2 a}{ }^{a}{ }^{a}$<smiles>O=C(CCl)c1ccccc1</smiles>

2a $(25 \mathrm{mM})$

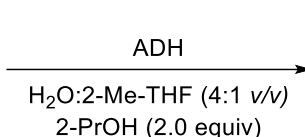

- $\mathrm{PrOH}(2.0$ equiv) $40^{\circ} \mathrm{C}, 24 \mathrm{~h}$ $220 \mathrm{rpm}$

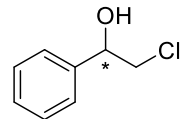

$3 a$

\begin{tabular}{|c|c|c|c|}
\hline Entry & $\mathrm{ADH}^{\mathrm{b}}$ & $3 \mathbf{a}(\%)^{\mathrm{c}}$ & $3 \mathbf{a} e e(\%)^{\mathrm{d}}$ \\
\hline 1 & RasADH & $<1$ & - \\
\hline 2 & $L b \mathrm{ADH}$ & 99 & $99(S)$ \\
\hline 3 & SyADH & $<1$ & - \\
\hline 4 & TeSADH & $<1$ & - \\
\hline 5 & ADH-T & $<1$ & - \\
\hline 6 & ADH-A & 98 & $>99(R)$ \\
\hline 7 & KRED-P1-A04 & $>99$ & $>99(S)$ \\
\hline 8 & KRED-P1-A12 & $>99$ & $99(S)$ \\
\hline 9 & KRED-P1-B02 & $>99$ & rac \\
\hline 10 & KRED-P1-B10 & 32 & n.d \\
\hline 11 & KRED-P1-B12 & $>99$ & $5(R)$ \\
\hline 12 & KRED-P1-C01 & 52 & $10(R)$ \\
\hline 13 & KRED-P1-H08 & $>99$ & $75(R)$ \\
\hline 14 & KRED-P2-B02 & $>99$ & $r a c$ \\
\hline 15 & KRED-P2-C02 & $>99$ & $20(R)$ \\
\hline 16 & KRED-P2-C11 & $>99$ & $77(S)$ \\
\hline 17 & KRED-P2-D03 & 17 & $60(R)$ \\
\hline 18 & KRED-P2-D11 & 77 & $50(R)$ \\
\hline 19 & KRED-P2-D12 & $<1$ & - \\
\hline 20 & KRED-P2-G03 & $>99$ & $70(S)$ \\
\hline 21 & KRED-P2-H07 & $>99$ & $>99(S)$ \\
\hline 22 & KRED-P3-B03 & 40 & $60(R)$ \\
\hline 23 & KRED-P3-G09 & 38 & $72(R)$ \\
\hline
\end{tabular}

a See below, Sections VII.2 to VII.6, for general procedures with all the tested enzymes.

b The coupled-substrate system employing 2-PrOH as cosubstrate was used for cofactor recycling purposes.

${ }^{\mathrm{c}}$ Product percentages were determined by GC analysis using calibration curves.

${ }^{\mathrm{d}}$ Enantiomeric excess values were determined by HPLC. The configuration of the major enantiomer appears in parentheses. $n . d$.: not determined. rac: racemate. 


\section{VII.2. Bioreduction of a-chloromethyl ketone 2a using RasADH}

Rather than the traditional glucose/glucose dehydrogenase enzyme-couple system used for the RasADH-catalysed reactions, in this contribution the coupled substrate system employing 2-PrOH as cosubstrate was employed. The general procedure is as follows: $\alpha-$ Chloro ketone 2a (2.3 mg, 0.015 mmol), 2-Me-THF (120 $\mu \mathrm{L}), 2-\mathrm{PrOH}(0.03 \mathrm{mmol}, 2.4$ $\mu \mathrm{L})$, a NADPH $10 \mathrm{mM}$ aqueous solution $(60 \mu \mathrm{L})$, distilled water $(420 \mu \mathrm{L})$ and lyophilised cells of $E$. coli overexpressing the RasADH (12 mg) were successively added to a $1.5 \mathrm{~mL}-$ Eppendorf tube. Then, the recipient was closed and kept under orbital shaking at $220 \mathrm{rpm}$ at $40{ }^{\circ} \mathrm{C}$ for $24 \mathrm{~h}$. After this time, the solution was extracted with EtOAc $(3 \times 0.5 \mathrm{~mL})$, the organic layers combined, dried over anhydrous $\mathrm{Na}_{2} \mathrm{SO}_{4}$ and filtered. The solution was concentrated, measuring then the reaction conversion and the enantiomeric excess of alcohol 3a by GC and HPLC analyses.

\section{VII.3. Bioreduction of a-chloromethyl ketone 2a using LbADH}

$\alpha$-Chloro ketone 2a (2.3 mg, $0.015 \mathrm{mmol}), 2-\mathrm{Me}-\mathrm{THF}(126 \mu \mathrm{L}), 2-\mathrm{PrOH}(0.03 \mathrm{mmol}, 2.4$ $\mu \mathrm{L}$ ), a NADPH $10 \mathrm{mM}$ aqueous solution $(60 \mu \mathrm{L}), \mathrm{agCl}_{2} 10 \mathrm{mM}$ aqueous solution (60

$\mu \mathrm{L})$, distilled water $(384 \mu \mathrm{L})$ and lyophilised cells of $E$. coli overexpressing $L b A D H(12$ $\mathrm{mg}$ ) were successively added to a $1.5 \mathrm{~mL}$-Eppendorf tube. Then, the recipient was closed and kept under orbital shaking at $220 \mathrm{rpm}$ at $40{ }^{\circ} \mathrm{C}$ for $24 \mathrm{~h}$. After this time, the solution was extracted with EtOAc $(3 \times 0.5 \mathrm{~mL})$, the organic layers combined, dried over anhydrous $\mathrm{Na}_{2} \mathrm{SO}_{4}$ and filtered. The solution was concentrated, measuring then the reaction conversion and the enantiomeric excess of alcohol 3a by GC and HPLC analyses.

\section{VII.4. Bioreduction of $\alpha$-chloromethyl ketone $2 a$ using SyADH, TeSADH and ADH-T}

$\alpha$-Chloro ketone 2a (2.3 mg, $0.015 \mathrm{mmol}), 2-\mathrm{Me}-\mathrm{THF}(120 \mu \mathrm{L}), 2-\mathrm{PrOH}(0.03 \mathrm{mmol}, 2.4$ $\mu \mathrm{L})$, a NADPH $10 \mathrm{mM}$ aqueous solution $(60 \mu \mathrm{L})$, distilled water $(420 \mu \mathrm{L})$ and lyophilised cells of the corresponding E. coli overexpressing ADH (12 mg) were successively added to a $1.5 \mathrm{~mL}$-Eppendorf tube. Then, the recipient was closed and kept under orbital shaking at $220 \mathrm{rpm}$ at $40{ }^{\circ} \mathrm{C}$ for $24 \mathrm{~h}$. After this time, the solution was extracted with EtOAc $(3 \mathrm{x}$ $0.5 \mathrm{~mL}$ ), the organic layers combined, dried over anhydrous $\mathrm{Na}_{2} \mathrm{SO}_{4}$ and filtered. The 
solution was concentrated, measuring then the reaction conversion and the enantiomeric excess of alcohol 3a by GC and HPLC analyses.

\section{VII.5. Bioreduction of a-chloromethyl ketone 2a using ADH-A}

$\alpha$-Chloro ketone 2a (2.3 mg, $0.015 \mathrm{mmol}), 2-\mathrm{Me}-\mathrm{THF}(120 \mu \mathrm{L}), 2-\mathrm{PrOH}(0.03 \mathrm{mmol}, 2.4$ $\mu \mathrm{L})$, a NADH $10 \mathrm{mM}$ aqueous solution $(60 \mu \mathrm{L})$, distilled water $(420 \mu \mathrm{L})$ and lyophilised cells of $E$. coli overexpressing $A D H-A(12 \mathrm{mg})$ were successively added to a $1.5 \mathrm{~mL}-$ Eppendorf tube. Then, the recipient was closed and kept under orbital shaking at $220 \mathrm{rpm}$ at $40{ }^{\circ} \mathrm{C}$ for $24 \mathrm{~h}$. After this time, the solution was extracted with EtOAc (3 x $\left.0.5 \mathrm{~mL}\right)$, the organic layers combined, dried over anhydrous $\mathrm{Na}_{2} \mathrm{SO}_{4}$ and filtered. The solution was concentrated, measuring then the reaction conversion and the enantiomeric excess of alcohol 3a by GC and HPLC analyses.

\section{VII.6. Bioreduction of a-chloromethyl ketone 2 a using commercial ADHs from Codexis}

The selected commercially available Codexis KRED $(1 \mathrm{mg})$ was added to a $1.5 \mathrm{~mL}$ Eppendorf tube containing $\alpha$-chloro ketone $\mathbf{2 a}$ (2.3 mg, $0.015 \mathrm{mmol}), 2-\mathrm{Me}-\mathrm{THF}$ (110 $\mu \mathrm{L}), 2-\mathrm{PrOH}(0.03 \mathrm{mmol}, 2.4 \mu \mathrm{L})$ and an aqueous solution containing $\mathrm{Na}_{3} \mathrm{PO}_{4}(128 \mathrm{mM})$, $\mathrm{MgSO}_{4}(1.7 \mathrm{mM})$ and $\mathrm{NADP}^{+}(1.1 \mathrm{mM})$ resulting in $\mathrm{pH} 7.0(435 \mu \mathrm{L})$. Then, the recipient was closed and kept under orbital shaking at $220 \mathrm{rpm}$ at $40{ }^{\circ} \mathrm{C}$ for $24 \mathrm{~h}$. After this time, the product was extracted with EtOAc $(3 \times 0.5 \mathrm{~mL})$, the organic layers combined, dried over anhydrous $\mathrm{Na}_{2} \mathrm{SO}_{4}$ and filtered. The solution was concentrated, measuring then the reaction conversion and the enantiomeric excess of alcohol 3a by GC and HPLC analyses. 
Table S6. Screening of different ADHs for the asymmetric bioreduction of $\mathbf{2} \mathbf{j}{ }^{a}$<smiles>O=C(CCl)c1ccccc1Cl</smiles>

2j

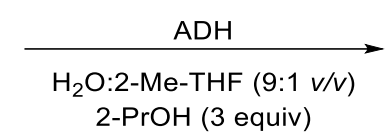

$40^{\circ} \mathrm{C}, 24 \mathrm{~h}$<smiles>OC(CCl)c1ccccc1Cl</smiles>

3j

\begin{tabular}{cccc}
\hline Entry & ADH & $\mathbf{3 j}(\%)^{\mathrm{b}}$ & $\mathbf{3 j} e e(\%)^{\mathrm{c}}$ \\
\hline 1 & LbADH & 44 & $87(S)$ \\
2 & TeSADH & $<1$ & - \\
3 & ADH-T & $<1$ & - \\
4 & ADH-A & 1 & - \\
\hline 5 & KRED-P1-A04 & $>99$ & $99(S)$ \\
6 & KRED-P1-A12 & $>99$ & $97(S)$ \\
7 & KRED-P1-B02 & $>99$ & $55(R)$ \\
8 & KRED-P1-B10 & $>99$ & $26(R)$ \\
9 & KRED-P1-B12 & $>99$ & $50(S)$ \\
10 & KRED-P1-C01 & $>99$ & $67(R)$ \\
11 & KRED-P1-H08 & $>99$ & $>99(R)$ \\
12 & KRED-P2-B02 & $>99$ & $98(R)$ \\
13 & KRED-P2-C02 & $>99$ & $97(R)$ \\
14 & KRED-P2-C11 & $>99$ & $r a c$ \\
15 & KRED-P2-D03 & $>99$ & $91(R)$ \\
16 & KRED-P2-D11 & 99 & $60(R)$ \\
17 & KRED-P2-D12 & $>99$ & $54(R)$
\end{tabular}

$\overline{{ }^{a}}$ Procedures already applied to ketone $\mathbf{2 a}$ were used for the bioreduction of $\mathbf{2} \mathbf{j}$, taking into account the mmol substrate/weight enzyme ratio.

${ }^{\mathrm{b}}$ Product percentages were determined by GC analysis using calibration curves.

${ }^{c}$ Enantiomeric excess values were determined by HPLC. The configuration of the major enantiomer appears in parentheses. rac: racemate. 


\section{Optimisation of the one-pot hydration-bioreduction cascade starting from alkyne 1a using $L b$ ADH}

Table S7. Optimisation of the hydration-bioreduction cascade starting from 1a using $\mathrm{Lb} \mathrm{ADH}$.

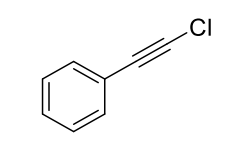

$1 \mathrm{a}$

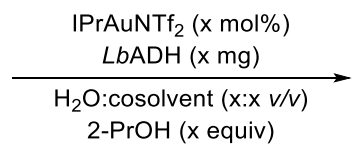

2-PrOH (x equiv)

$\mathrm{MgCl}_{2}(1 \mathrm{mM})$

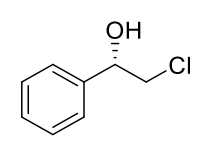

(S)-3a

\begin{tabular}{|c|c|c|c|c|c|c|c|c|c|c|c|c|}
\hline Entry & $\begin{array}{l}\text { LbADH } \\
\quad(\mathrm{mg})\end{array}$ & $\begin{array}{c}\operatorname{IPrAuNTf}_{2} \\
(\mathrm{~mol} \%)\end{array}$ & $\begin{array}{l}\text { 2-PrOH } \\
\text { (equiv) }\end{array}$ & $\begin{array}{c}\text { NADPH } \\
(\mathrm{mM})\end{array}$ & Medium & $\mathrm{T}\left({ }^{\circ} \mathrm{C}\right)$ & Stirring & $\begin{array}{c}1 \mathbf{a} \\
(\%)^{\mathrm{a}}\end{array}$ & $\begin{array}{c}\mathbf{2 a} \\
(\%)^{\mathrm{a}}\end{array}$ & $\begin{array}{c}\text { By- } \\
\text { products } \\
(\%)^{\mathrm{a}}\end{array}$ & $\begin{array}{c}\mathbf{3 a} \\
(\%)^{\mathrm{a}}\end{array}$ & $\begin{array}{c}(S)-\mathbf{3 a} \\
e e(\%)^{\mathrm{b}}\end{array}$ \\
\hline 1 & 46 & 5 & 2 & 1 & $4: 1\left(\mathrm{H}_{2} \mathrm{O}: \mathrm{THF}\right)$ & 40 & $220 \mathrm{rpm}^{\mathrm{c}}$ & 28 & $<1$ & 9 & 63 & $>99$ \\
\hline 2 & 20 & 5 & 2 & 1 & 4:1 ( $\left.\mathrm{H}_{2} \mathrm{O}: \mathrm{THF}\right)$ & 40 & $220 \mathrm{rpm}^{\mathrm{c}}$ & 20 & $<1$ & 9 & 71 & $>99$ \\
\hline 3 & 20 & 5 & 3 & 1 & 4:1 ( $\left.\mathrm{H}_{2} \mathrm{O}: \mathrm{THF}\right)$ & 40 & Magnetic & 3 & $<1$ & 6 & $91(85)$ & $>99$ \\
\hline 4 & 20 & 6 & 3 & 1 & 4:1 ( $\left.\mathrm{H}_{2} \mathrm{O}: \mathrm{THF}\right)$ & 40 & Magnetic & $<1$ & 1 & 7 & $92(86)$ & $>99$ \\
\hline 5 & 20 & 6 & 3 & 1 & $4: 1\left(\mathrm{H}_{2} \mathrm{O}: \mathrm{THF}\right)$ & 45 & Magnetic & $<1$ & $<1$ & 7 & $93(85)$ & $>99$ \\
\hline 6 & 20 & 6 & 3 & 1 & $4: 1\left(\mathrm{H}_{2} \mathrm{O}: 2 \mathrm{Me}-\mathrm{THF}\right)$ & 40 & Magnetic & $<1$ & $<1$ & 6 & $94(86)$ & $>99$ \\
\hline 7 & 20 & 6 & 2 & 1 & $9: 1\left(\mathrm{H}_{2} \mathrm{O}: 2 \mathrm{Me}-\mathrm{THF}\right)$ & 40 & Magnetic & $<1$ & $<1$ & 4 & $96(87)$ & $>99$ \\
\hline 8 & 20 & 6 & 2 & 2 & $9: 1\left(\mathrm{H}_{2} \mathrm{O}: 2 \mathrm{Me}-\mathrm{THF}\right)$ & 40 & Magnetic & $<1$ & $<1$ & 4 & $96(86)$ & $>99$ \\
\hline 9 & 20 & 6 & 2.5 & 1 & 9:1 $\left(\mathrm{H}_{2} \mathrm{O}: 2 \mathrm{Me}-\mathrm{THF}\right)$ & 40 & Magnetic & $<1$ & $<1$ & 7 & 93 & $>99$ \\
\hline
\end{tabular}

a Product percentages were determined by GC analysis using calibration curves. Isolated yields of ( $S)$-3a in parentheses.

${ }^{\mathrm{b}}$ Enantiomeric excess values were determined by HPLC.

${ }^{c}$ Orbital stirring. 


\section{Study of the one-pot hydration-bioreduction cascade for 2a: substrate concentration influence}

Table S8. Dependence on the substrate concentration for the gold-catalysed hydration of alkyne 1a using $\operatorname{IPrAuNTf}_{2}$.


- $\mathrm{PrOH}(2$ equiv)

$2 a$

\begin{tabular}{ccccc}
\hline Entry & $\mathbf{1 a}(\mathrm{mM})$ & $\mathbf{1 a}(\%)^{\mathrm{a}}$ & $\mathbf{2 a}(\%)^{\mathrm{a}}$ & ${\text { By-products }(\%)^{\mathrm{a}}}^{\mathrm{a}}$ \\
\hline 1 & 100 & $<1$ & $99(93)$ & 1 \\
2 & 150 & $<1$ & $99(91)$ & 1 \\
3 & 200 & $<1$ & $99(93)$ & 1 \\
4 & 250 & $<1$ & $99(93)$ & 1 \\
5 & 300 & $<1$ & $99(92)$ & 1 \\
\hline
\end{tabular}

a Product percentages were determined by GC analysis using calibration curves. Isolated yields of ketone 2a in parentheses.

Table S9. Dependence on the substrate concentration for the hydration-bioreduction cascade for 1a using $\operatorname{IPrAuNTf}_{2}$ and $\mathrm{LbADH}$.


Magnetic stirring

\begin{tabular}{ccccccc}
\hline Entry & $\mathbf{1 a}(\mathrm{mM})$ & $\mathbf{1 a}(\%)^{\mathrm{a}}$ & $\mathbf{2 a}(\%)^{\mathrm{a}}$ & $\begin{array}{c}\text { By-products } \\
(\%)^{\mathrm{a}}\end{array}$ & $\mathbf{3 a}(\%)^{\mathrm{a}}$ & $\begin{array}{c}(S)-3 \mathbf{a} e e \\
(\%)^{\mathrm{b}}\end{array}$ \\
\hline 1 & 100 & $<1$ & $<1$ & 4 & $96(87)$ & $>99$ \\
2 & 150 & $<1$ & $<1$ & 6 & $94(84)$ & $>99$ \\
3 & 200 & $<1$ & $<1$ & 11 & $89(75)$ & $>99$ \\
4 & 250 & $<1$ & $<1$ & 16 & $84(69)$ & $>99$ \\
\hline
\end{tabular}

${ }^{a}$ Product percentages were determined by GC analysis using calibration curves. Isolated yields of $(S)-\mathbf{3 a}$ in parentheses.

${ }^{\mathrm{b}}$ Enantiomeric excess values were determined by HPLC. 


\section{$\mathrm{X}$. Scope of the one-pot cascade process and full characterisation of alkynes 3a-I}

Table S10. Scope of the concurrent cascade gold-catalysed hydration and stereoselective bioreduction of alkynes 1a-I.



$$
\begin{aligned}
& \begin{array}{ccc}
1 \mathrm{a}-\mathrm{I} & \mathrm{H}_{2} \mathrm{O}: 2 \mathrm{Me}-\mathrm{THF}(9: 1 \mathrm{v} / \mathrm{V}) & (R) \text { - or }(S) \text {-3a-I } \\
0.1 \mathrm{mmol} & 40^{\circ} \mathrm{C}, 24 \mathrm{~h} &
\end{array} \\
& 0.1 \mathrm{mmol} \quad 40^{\circ} \mathrm{C}
\end{aligned}
$$

\begin{tabular}{|c|c|c|c|c|c|c|c|c|c|}
\hline Entry & Compound & $\mathrm{R}^{1}$ & $\mathrm{R}^{2}$ & Enzyme & $1(\%)^{\mathrm{a}}$ & $2(\%)^{\mathrm{a}}$ & By-products $(\%)^{\mathrm{a}}$ & $\mathbf{3}(\%)^{\mathrm{a}}$ & 3 ee $(\%)^{\mathrm{b}}$ \\
\hline 1 & $1 \mathbf{a}$ & $\mathrm{Ph}$ & $\mathrm{Cl}$ & $L b \mathrm{ADH}$ & $<1$ & $<1$ & 4 & $96(86)$ & $>99(S)$ \\
\hline 2 & $\mathbf{1 a}$ & $\mathrm{Ph}$ & $\mathrm{Cl}$ & ADH-A & $<1$ & $<1$ & 6 & $94(85)$ & $>99(R)$ \\
\hline 3 & $1 b$ & $4-\mathrm{F}-\mathrm{C}_{6} \mathrm{H}_{4}$ & $\mathrm{Cl}$ & LbADH & $<1$ & 1 & 4 & $95(84)$ & $>99(S)$ \\
\hline 4 & $1 b$ & $4-\mathrm{F}-\mathrm{C}_{6} \mathrm{H}_{4}$ & $\mathrm{Cl}$ & ADH-A & $<1$ & $<1$ & 5 & $95(82)$ & $>99(R)$ \\
\hline 5 & 1c & 4-Cl- $\mathrm{C}_{6} \mathrm{H}_{4}$ & $\mathrm{Cl}$ & $L b A D H$ & $<1$ & $<1$ & 6 & $94(86)$ & $>99(S)$ \\
\hline 6 & 1c & 4-Cl- $\mathrm{C}_{6} \mathrm{H}_{4}$ & $\mathrm{Cl}$ & ADH-A & $<1$ & $<1$ & 8 & $92(80)$ & $>99(R)$ \\
\hline 7 & $1 d$ & $4-\mathrm{Br}-\mathrm{C}_{6} \mathrm{H}_{4}$ & $\mathrm{Cl}$ & $L b A D H$ & $<1$ & $<1$ & 8 & $92(83)$ & $99(S)$ \\
\hline 8 & 1d & $4-\mathrm{Br}-\mathrm{C}_{6} \mathrm{H}_{4}$ & $\mathrm{Cl}$ & ADH-A & $<1$ & $<1$ & 8 & $92(81)$ & $99(R)$ \\
\hline 9 & 1e & $4-\mathrm{Me}-\mathrm{C}_{6} \mathrm{H}_{4}$ & $\mathrm{Cl}$ & $L b \mathrm{ADH}$ & $<1$ & $<1$ & 6 & $93(82)$ & $99(S)$ \\
\hline 10 & 1e & $4-\mathrm{Me}-\mathrm{C}_{6} \mathrm{H}_{4}$ & $\mathrm{Cl}$ & ADH-A & $<1$ & $<1$ & 10 & $90(77)$ & $99(R)$ \\
\hline 11 & 1f & $4-\mathrm{OMe}-\mathrm{C}_{6} \mathrm{H}_{4}$ & $\mathrm{Cl}$ & $L b A D H$ & $<1$ & $<1$ & 9 & $91(79)$ & $>99(S)$ \\
\hline 12 & 1f & $4-\mathrm{OMe}-\mathrm{C}_{6} \mathrm{H}_{4}$ & $\mathrm{Cl}$ & ADH-A & $<1$ & $<1$ & 16 & $84(70)$ & $99(R)$ \\
\hline
\end{tabular}


Table S10 continuation.

\begin{tabular}{|c|c|c|c|c|c|c|c|c|c|}
\hline Entry & Compound & $\mathrm{R}^{1}$ & $\mathrm{R}^{2}$ & Enzyme & $\mathbf{1}(\%)^{\mathrm{a}}$ & $2(\%)^{\mathrm{a}}$ & By-products $(\%)^{\mathrm{a}}$ & $\mathbf{3}(\%)^{\mathrm{a}}$ & 3 ee $(\%)^{\mathrm{b}}$ \\
\hline 13 & $1 g$ & $3-\mathrm{Cl}-\mathrm{C}_{6} \mathrm{H}_{4}$ & $\mathrm{Cl}$ & $L b \mathrm{ADH}$ & 3 & 2 & 7 & $88(77)$ & $>99(S)$ \\
\hline 14 & $1 \mathrm{~g}$ & $3-\mathrm{Cl}-\mathrm{C}_{6} \mathrm{H}_{4}$ & $\mathrm{Cl}$ & ADH-A & 1 & 2 & 11 & $86(75)$ & $99(R)$ \\
\hline 15 & $1 \mathrm{~h}$ & $3-\mathrm{OMe}-\mathrm{C}_{6} \mathrm{H}_{4}$ & $\mathrm{Cl}$ & LbADH & $<1$ & 3 & 7 & $90(81)$ & $>99(S)$ \\
\hline 16 & $1 \mathrm{~h}$ & $3-\mathrm{OMe}-\mathrm{C}_{6} \mathrm{H}_{4}$ & $\mathrm{Cl}$ & $\mathrm{ADH}-\mathrm{A}$ & $<1$ & 1 & 13 & $86(76)$ & $>99(R)$ \\
\hline 17 & $\mathbf{1 i}$ & $3,4-\mathrm{Cl}_{2}-\mathrm{C}_{6} \mathrm{H}_{3}$ & $\mathrm{Cl}$ & $L b A D H$ & 7 & 1 & 8 & $84(71)$ & $99(S)$ \\
\hline 18 & $\mathbf{1 i}$ & $3,4-\mathrm{Cl}_{2}-\mathrm{C}_{6} \mathrm{H}_{3}$ & $\mathrm{Cl}$ & $\mathrm{ADH}-\mathrm{A}$ & 5 & 3 & 14 & $78(65)$ & $>99(R)$ \\
\hline 19 & $\mathbf{1 j}$ & $2-\mathrm{Cl}-\mathrm{C}_{6} \mathrm{H}_{4}$ & $\mathrm{Cl}$ & KRED-P1-A04 & $<1$ & 74 & 6 & 20 & $99(S)$ \\
\hline 20 & $\mathbf{1 j}$ & $2-\mathrm{Cl}-\mathrm{C}_{6} \mathrm{H}_{4}$ & $\mathrm{Cl}$ & KRED-P1-H08 & $<1$ & 76 & 7 & 17 & $>99(R)$ \\
\hline 21 & $\mathbf{1} \mathbf{j}^{\mathrm{c}}$ & $2-\mathrm{Cl}-\mathrm{C}_{6} \mathrm{H}_{4}$ & $\mathrm{Cl}$ & KRED-P1-A04 & $<1$ & 5 & 16 & 79 (69) & $99(S)$ \\
\hline 22 & $\mathbf{1} \mathbf{j}^{\mathrm{c}}$ & $2-\mathrm{Cl}-\mathrm{C}_{6} \mathrm{H}_{4}$ & $\mathrm{Cl}$ & KRED-P1-H08 & $<1$ & 4 & 18 & $78(66)$ & $>99(R)$ \\
\hline 23 & $\mathbf{1 k}$ & $\mathrm{C}_{6} \mathrm{H}_{13}$ & $\mathrm{Cl}$ & $L b A D H$ & $<1$ & $<1$ & 2 & $98(83)$ & $>99(S)$ \\
\hline 24 & $\mathbf{1 k}$ & $\mathrm{C}_{6} \mathrm{H}_{13}$ & $\mathrm{Cl}$ & ADH-A & $<1$ & $<1$ & 4 & $96(84)$ & $99(R)$ \\
\hline 25 & $\mathbf{1 k}$ & $\mathrm{C}_{6} \mathrm{H}_{13}$ & $\mathrm{Cl}$ & ADH-T & $<1$ & 2 & 4 & $94(81)$ & $>99(R)$ \\
\hline 26 & $\mathbf{1 k}$ & $\mathrm{C}_{6} \mathrm{H}_{13}$ & $\mathrm{Cl}$ & TeSADH & $<1$ & 58 & 5 & 37 & n.d. \\
\hline 27 & 11 & $\mathrm{Ph}$ & $\mathrm{Br}$ & LbADH & $<1$ & $<1$ & 9 & $91(80)$ & $99(S)$ \\
\hline
\end{tabular}

a Product percentages were determined by GC analysis using calibration curves. Isolated yields of 3a-l appear in parentheses.

${ }^{\mathrm{b}}$ Enantiomeric excess values were determined by HPLC, except for the aliphatic alcohol 3k that GC analyses was required.

${ }^{c}$ A 85:10:5 system $\left(\mathrm{H}_{2} \mathrm{O}: 2-\mathrm{Me}-\mathrm{THF}: 2-\mathrm{PrOH}\right)$ was employed, which is equivalent to the use of 10.5 equivalents of 2-PrOH. 

to obtain enantioenriched alcohols $3 a-l$

The corresponding alkyne 1a-l $(0.1 \mathrm{mmol}), 2-\mathrm{Me}-\mathrm{THF}(100 \mu \mathrm{L})$, distilled water (705 $\mu \mathrm{L}), 2-\operatorname{PrOH}\left(15.3 \mu \mathrm{L}, 2\right.$ equiv), $\operatorname{IPrAuNTf}_{2}(5.3 \mathrm{mg}, 0.006 \mathrm{mmol}, 6.0 \mathrm{~mol} \%$ ), a NADH aqueous solution $(100 \mu \mathrm{L}, 10 \mathrm{mM}), \mathrm{MgCl}_{2}$ aqueous solution $(100 \mu \mathrm{L}, 10$ $\mathrm{mM}$ ) and overexpressed E. coli/LbADH (20 mg) were placed into a glass vial. The mixture was kept under magnetic stirring at $40{ }^{\circ} \mathrm{C}$ for $24 \mathrm{~h}$, and after this time, the solution was extracted with EtOAc $(5 \times 1 \mathrm{~mL})$, the organic layers combined, dried over anhydrous $\mathrm{Na}_{2} \mathrm{SO}_{4}$ and filtered. The solvent was evaporated under reduced pressure, measuring then the conversions into the alcohols 3a-l by GC analysis, while the enantiomeric excess values were calculated by HPLC o GC (see Section XIV). Alcohols 3a-l were purified by column chromatography on silica gel.

\section{X.II. Experimental protocol for the one-pot cascade reaction employing E. coli/ADH-} A to obtain enantioenriched alcohols $3 a-k$

The corresponding alkyne 1a-k $(0.1 \mathrm{mmol}), 2-\mathrm{Me}-\mathrm{THF}(100 \mu \mathrm{L})$, distilled water $(800$ $\mu \mathrm{L}), 2-\mathrm{PrOH}$ ( $23 \mu \mathrm{L}, 3$ equiv), $\operatorname{IPrAuNTf}_{2}(5.3 \mathrm{mg}, 0.006 \mathrm{mmol}, 6.0 \mathrm{~mol} \%)$, a NADH aqueous solution $(100 \mu \mathrm{L}, 10 \mathrm{mM})$, and overexpressed E. coli/ADH-A (20 mg) were placed into a glass vial. The mixture was kept under magnetic stirring at $40{ }^{\circ} \mathrm{C}$ for 24 $\mathrm{h}$, and after this time, the solution was extracted with EtOAc $(5 \times 1 \mathrm{~mL})$, the organic layers combined, dried over anhydrous $\mathrm{Na}_{2} \mathrm{SO}_{4}$ and filtered. The solvent was evaporated under reduced pressure, measuring then the conversions into the alcohols 3a-k by GC analysis, while the enantiomeric excess values were calculated by HPLC o GC (see Section XIV). Alcohols 3a-k were purified by column chromatography on silica gel.

\section{X.III. Experimental protocol for the one-pot cascade reaction employing Codexis}

\section{KREDs to obtain alcohol $3 j$}

Alkyne 1j (20.5 mg, $0.1 \mathrm{mmol})$, 2-Me-THF (100 $\mu \mathrm{L})$, distilled water (655 $\mu \mathrm{L}), 2$ PrOH (50 $\mu \mathrm{L}, 10.6$ equiv), IPrAuNTf 2 (5.3 mg, $0.006 \mathrm{mmol}, 6.0 \mathrm{~mol} \%$ ), a NADPH aqueous solution $(100 \mu \mathrm{L}, 10 \mathrm{mM}), \mathrm{MgCl}_{2}$ aqueous solution $(100 \mu \mathrm{L}, 10 \mathrm{mM})$ and 
the corresponding KRED (16 mg) were placed into a glass vial. The mixture was kept under magnetic stirring at $40{ }^{\circ} \mathrm{C}$ for $24 \mathrm{~h}$, and after this time, the solution was extracted with EtOAc ( 5 x $1 \mathrm{~mL})$, the organic layers combined, dried over anhydrous $\mathrm{Na}_{2} \mathrm{SO}_{4}$ and filtered. The solvent was evaporated under reduced pressure, measuring then the conversions into the alcohol $\mathbf{3 j}$ by GC analysis, while the enantiomeric excess values were calculated by HPLC. Alcohol $\mathbf{3 j}$ was purified by column chromatography on silica gel.

X.IV. Experimental protocol for the one-pot cascade reaction employing E. coli/ADHT to obtain alcohol $3 \mathrm{k}$

Alkyne 1k (14.5 mg, $0.1 \mathrm{mmol}), 2-\mathrm{Me}-\mathrm{THF}(100 \mu \mathrm{L})$, distilled water $(800 \mu \mathrm{L}), 2$ PrOH (23 $\mu \mathrm{L}, 3$ equiv), IPrAuNTf 2 (5.3 mg, $0.006 \mathrm{mmol}, 6.0 \mathrm{~mol} \%$ ), a NADPH aqueous solution $(100 \mu \mathrm{L}, 10 \mathrm{mM})$, and overexpressed E. coli/ADH-T (20 mg) were placed into a glass vial. The mixture was kept under magnetic stirring at $40{ }^{\circ} \mathrm{C}$ for 24 $\mathrm{h}$, and after this time, the solution was extracted with EtOAc $(5 \times 1 \mathrm{~mL})$, the organic layers combined, dried over anhydrous $\mathrm{Na}_{2} \mathrm{SO}_{4}$ and filtered. The solvent was evaporated under reduced pressure, measuring then the conversions into the alcohol 3k and the enantiomeric excess by GC analysis. Alcohol 3k was purified by column chromatography on silica gel.

Full characterisations of alcohols 3a-k appear below, while for the obtained specific rotation values see Section XV (Table S17):

2-Chloro-1-phenylethan-1-ol (3a): Colorless oil. $R_{\mathrm{f}}$ (10\% EtOAc/hexane): 0.29 . IR: $v$ 3367, 2005, 2989, 1275, 1260 and $767 \mathrm{~cm}^{-1} .{ }^{1} \mathrm{H}-\mathrm{NMR}\left(300.13 \mathrm{MHz}, \mathrm{CDCl}_{3}\right): \delta$ 7.41-7.34 $(m, 5 \mathrm{H}), 4.86(m, 1 \mathrm{H}), 3.71(d d, J=11.3,3.9 \mathrm{~Hz}, 1 \mathrm{H}), 3.63(d d, J=11.1,8.2 \mathrm{~Hz}, 1 \mathrm{H})$, 3.32 (br s, 1H). ${ }^{13} \mathrm{C}-\mathrm{NMR}\left(75.5 \mathrm{MHz}, \mathrm{CDCl}_{3}\right): \delta 140.2(\mathrm{C}), 128.7(2 \mathrm{CH}), 128.5(\mathrm{CH})$, $126.2(2 \mathrm{CH}), 74.1(\mathrm{CH}), 50.6\left(\mathrm{CH}_{2}\right) .\left(\mathrm{ESI}^{+}, \mathrm{m} / \mathrm{z}\right)$ : calcd for $\left(\mathrm{C}_{8} \mathrm{H}_{9} \mathrm{ClNaO}\right)^{+}(\mathrm{M}+\mathrm{Na})^{+}$: 179.0234; found 179.0235.

2-Chloro-1-(4-fluorophenyl)ethan-1-ol (3b): Colorless oil. $R_{\mathrm{f}}$ (10\% EtOAc/hexane): 0.31. IR: v 3377, 3005, 2989, 1273, 1267 and $765 \mathrm{~cm}^{-1} .{ }^{1} \mathrm{H}-\mathrm{NMR}\left(300.13 \mathrm{MHz}, \mathrm{CDCl}_{3}\right)$ : $\delta 7.37(m, 2 \mathrm{H}), 7.06(m, 2 \mathrm{H}), 4.89(d t, J=8.7,3.4 \mathrm{~Hz}, 1 \mathrm{H}), 3.71(d d, J=11.2,3.6 \mathrm{~Hz}$, 
$1 \mathrm{H}), 3.61(d d, J=11.3,8.6 \mathrm{~Hz}, 1 \mathrm{H}), 2.72(m, 1 \mathrm{H}) .{ }^{13} \mathrm{C}-\mathrm{NMR}\left(75.5 \mathrm{MHz}, \mathrm{CDCl}_{3}\right): \delta 162.7$ $(d, J=247.0 \mathrm{~Hz}, \mathrm{C}), 135.7(\mathrm{C}), 127.8(d, J=8.3 \mathrm{~Hz}, 2 \mathrm{CH}), 115.6(d, J=21.0 \mathrm{~Hz}, 2 \mathrm{CH})$, $73.4(\mathrm{CH}), 50.8\left(\mathrm{CH}_{2}\right) .{ }^{19} \mathrm{~F}-\mathrm{RMN}\left(282 \mathrm{MHz}, \mathrm{CDCl}_{3}\right): \delta-113.50 .\left(\mathrm{ESI}^{+}, \mathrm{m} / \mathrm{z}\right)$ : calcd for $\left(\mathrm{C}_{8} \mathrm{H}_{8} \mathrm{ClFNaO}\right)^{+}(\mathrm{M}+\mathrm{Na})^{+}:$197.0140; found 197.0144.

2-Chloro-1-(4-chlorophenyl)ethan-1-ol (3c): Colorless oil. $R_{\mathrm{f}}(10 \%$ EtOAc/hexane): 0.23. IR: $v$ 3368, 3004, 2988, 1265, 1275 and $755 \mathrm{~cm}^{-1} .{ }^{1} \mathrm{H}-\mathrm{NMR}\left(300.13 \mathrm{MHz}, \mathrm{CDCl}_{3}\right)$ : $\delta$ 7.38-7.31 (m, 4H), $4.89(d t, J=8.6,3.4 \mathrm{~Hz}, 1 \mathrm{H}), 3.72(d d, J=11.3,3.4 \mathrm{~Hz}, 1 \mathrm{H}), 3.61$ $(d d, J=11.3,8.6 \mathrm{~Hz}, 1 \mathrm{H}), 2.69(d, J=3.3 \mathrm{~Hz}, 1 \mathrm{H}) .{ }^{13} \mathrm{C}-\mathrm{NMR}\left(75.5 \mathrm{MHz}, \mathrm{CDCl}_{3}\right): \delta$ $138.4(\mathrm{C}), 134.2(\mathrm{C}), 128.9(2 \mathrm{CH}), 127.5(2 \mathrm{CH}), 73.4(\mathrm{CH}), 50.7\left(\mathrm{CH}_{2}\right) .\left(\mathrm{ESI}^{+}, \mathrm{m} / \mathrm{z}\right)$ : calcd for $\left(\mathrm{C}_{8} \mathrm{H}_{8} \mathrm{Cl}_{2} \mathrm{NaO}\right)^{+}(\mathrm{M}+\mathrm{Na})^{+}:$212.9844; found 212.9848 .

2-Chloro-1-(4-bromophenyl)ethan-1-ol (3d): Colorless oil. $R_{\mathrm{f}}(10 \%$ EtOAc/hexane): 0.21. IR: v 3366, 3005, 2989, 1275, 1267 and $763 \mathrm{~cm}^{-1} .{ }^{1} \mathrm{H}-\mathrm{NMR}\left(300.13 \mathrm{MHz}, \mathrm{CDCl}_{3}\right)$ : $\delta 7.51(m, 2 \mathrm{H}), 7.27(m, 2 \mathrm{H}), 4.87(d t, J=8.6,3.1 \mathrm{~Hz}, 1 \mathrm{H}), 3.72(d d, J=11.2,3.6 \mathrm{~Hz}$, $1 \mathrm{H}), 3.60(d d, J=11.3,8.5 \mathrm{~Hz}, 1 \mathrm{H}), 2.72(d, J=3.3 \mathrm{~Hz}, 1 \mathrm{H}) .{ }^{13} \mathrm{C}-\mathrm{NMR}(75.5 \mathrm{MHz}$, $\left.\mathrm{CDCl}_{3}\right): \delta 138.9(\mathrm{C}), 131.8(2 \mathrm{CH}), 127.8(2 \mathrm{CH}), 122.4(\mathrm{C}), 73.4(\mathrm{CH}), 50.6\left(\mathrm{CH}_{2}\right) .\left(\mathrm{ESI}^{+}\right.$, $\mathrm{m} / \mathrm{z})$ : calcd for $\left(\mathrm{C}_{8} \mathrm{H}_{8} \mathrm{BrClNaO}\right)^{+}(\mathrm{M}+\mathrm{Na})^{+}:$256.9339; found 256.9339 .

2-Chloro-1-(p-tolyl)ethan-1-ol (3e): Colorless oil. $R_{\mathrm{f}}(20 \%$ EtOAc/hexane): 0.52. IR: v 3368, 3005, 2987, 1267, 1260 and $758 \mathrm{~cm}^{-1} .{ }^{1} \mathrm{H}-\mathrm{NMR}\left(300.13 \mathrm{MHz}, \mathrm{CDCl}_{3}\right): \delta 7.30(\mathrm{~m}$, 2H), $7.22(m, 2 \mathrm{H}), 4.89(d t, J=8.7,3.4 \mathrm{~Hz}, 1 \mathrm{H}), 3.75(d d, J=11.2,3.6 \mathrm{~Hz}, 1 \mathrm{H}), 3.66$ $(d d, J=11.2,8.6 \mathrm{~Hz}, 1 \mathrm{H}), 2.69(d, J=3.3 \mathrm{~Hz}, 1 \mathrm{H}), 2.38(s, 3 \mathrm{H}) .{ }^{13} \mathrm{C}-\mathrm{NMR}(75.5 \mathrm{MHz}$, $\left.\mathrm{CDCl}_{3}\right): \delta 138.3(\mathrm{C}), 137.0(\mathrm{C}), 129.4(2 \mathrm{CH}), 126.0(2 \mathrm{CH}), 73.9(\mathrm{CH}), 50.9\left(\mathrm{CH}_{2}\right), 21.7$ $\left(\mathrm{CH}_{3}\right) .\left(\mathrm{ESI}^{+}, \mathrm{m} / \mathrm{z}\right)$ : calcd for $\left(\mathrm{C}_{9} \mathrm{H}_{11} \mathrm{ClNaO}\right)^{+}(\mathrm{M}+\mathrm{Na})^{+}: 193.0391$; found 193.0392 .

2-Chloro-1-(4-methoxyphenyl)ethan-1-ol (3f): Colorless oil. $R_{\mathrm{f}}(10 \%$ EtOAc/hexane): 0.20. IR: v 3388, 3005, 2988, 1267, 1260 and $766 \mathrm{~cm}^{-1} .{ }^{1} \mathrm{H}-\mathrm{NMR}\left(300.13 \mathrm{MHz}, \mathrm{CDCl}_{3}\right)$ : $\delta 7.29(m, 2 \mathrm{H}), 6.91(m, 2 \mathrm{H}), 4.84(d t, J=8.6,3.4 \mathrm{~Hz}, 1 \mathrm{H}), 3.81(s, 3 \mathrm{H}), 3.70(d d, J=$ 11.1, 3.8 Hz, 1H), $3.63(d d, J=11.3,8.6 \mathrm{~Hz}, 1 \mathrm{H}), 2.68(d, J=3.1 \mathrm{~Hz}, 1 \mathrm{H}) .{ }^{13} \mathrm{C}-\mathrm{NMR}$ (75.5 MHz, $\left.\mathrm{CDCl}_{3}\right): \delta 159.7(\mathrm{C}), 132.1(\mathrm{C}), 127.3(2 \mathrm{CH}), 114.1(2 \mathrm{CH}), 73.7(\mathrm{CH}), 55.3$ $\left(\mathrm{CH}_{3}\right), 50.9\left(\mathrm{CH}_{2}\right)$. $\left(\mathrm{ESI}^{+}, \mathrm{m} / \mathrm{z}\right)$ : calcd for $\left(\mathrm{C}_{9} \mathrm{H}_{11} \mathrm{ClNaO}_{2}\right)^{+}(\mathrm{M}+\mathrm{Na})^{+}: 209.0340$; found 209.0342 . 
2-Chloro-1-(3-chlorophenyl)ethan-1-ol (3g): Colorless oil. $R_{\mathrm{f}}(30 \%$ EtOAc/hexane): 0.70. ${ }^{1} \mathrm{H}-\mathrm{NMR}\left(300.13 \mathrm{MHz}, \mathrm{CDCl}_{3}\right): \delta 7.41(m, 1 \mathrm{H}), 7.30(m, 3 \mathrm{H}), 4.88(d d, J=8.5$, 3.5, 1H), 3.77-3.59 (m, 2H), 2.77 (br s, 1H). ${ }^{13} \mathrm{C}-\mathrm{NMR}\left(75.5 \mathrm{MHz}, \mathrm{CDCl}_{3}\right): \delta 141.9(\mathrm{C})$, $136.6(\mathrm{C}), 129.9(\mathrm{CH}), 128.6(\mathrm{CH}), 126.3(\mathrm{CH}), 124.3(\mathrm{CH}), 73.4(\mathrm{CH}), 50.6\left(\mathrm{CH}_{2}\right)$.

2-Chloro-1-(3-methoxyphenyl)ethan-1-ol (3h): Colorless oil. $R_{\mathrm{f}}(15 \%$ EtOAc/hexane): 0.37. ${ }^{1} \mathrm{H}-\mathrm{NMR}\left(300.13 \mathrm{MHz}, \mathrm{CDCl}_{3}\right): \delta 7.32\left(t_{\text {apparent }}, J=8.1 \mathrm{~Hz}, 1 \mathrm{H}\right), 6.96(m, 2 \mathrm{H}), 6.88$ $(d d d, J=8.2,2.5,0.9 \mathrm{~Hz}, 1 \mathrm{H}), 4.88(d d, J=8.6,3.5 \mathrm{~Hz}, 1 \mathrm{H}), 3.83(s, 3 \mathrm{H}), 3.75(d d, J=$ 11.2, $3.5 \mathrm{~Hz}, 1 \mathrm{H}), 3.65(d d, J=11.2,8.6 \mathrm{~Hz}, 1 \mathrm{H}) .{ }^{13} \mathrm{C}-\mathrm{NMR}\left(75.5 \mathrm{MHz}, \mathrm{CDCl}_{3}\right): \delta 159.8$ (C), $141.6(\mathrm{C}), 129.7(\mathrm{CH}), 118.3(\mathrm{CH}), 113.9(\mathrm{CH}), 111.6(\mathrm{CH}), 74.0(\mathrm{CH}), 55.3\left(\mathrm{CH}_{3}\right)$, $50.8\left(\mathrm{CH}_{2}\right)$.

2-Chloro-1-(3,4-dichlorophenyl)ethan-1-ol (3i): Colorless $\quad$ oil. $R_{\mathrm{f}} \quad(20 \%$ EtOAc/hexane): 0.35. IR: $v$ 3368, 3005, 2989, 1275, 1261 and $764 \mathrm{~cm}^{-1} .{ }^{1} \mathrm{H}-\mathrm{NMR}$ $\left(300.13 \mathrm{MHz}, \mathrm{CDCl}_{3}\right): \delta 7.52(d, J=2.1 \mathrm{~Hz}, 1 \mathrm{H}), 7.45(d, J=8.2 \mathrm{~Hz}, 1 \mathrm{H}), 7.22(d d d, J$ $=8.3,2.1,0.6 \mathrm{~Hz}, 1 \mathrm{H}), 4.88(d t, J=8.5,3.5 \mathrm{~Hz}, 1 \mathrm{H}), 3.73(d d, J=11.3,3.5 \mathrm{~Hz}, 1 \mathrm{H})$, $3.60(d d, J=11.3,8.5 \mathrm{~Hz}, 1 \mathrm{H}), 2.70(d, J=3.4 \mathrm{~Hz}, 1 \mathrm{H}) .{ }^{13} \mathrm{C}-\mathrm{NMR}\left(75.5 \mathrm{MHz}, \mathrm{CDCl}_{3}\right)$ : $\delta 140.0(\mathrm{C}), 132.9(\mathrm{C}), 132.4(\mathrm{C}), 130.6(\mathrm{CH}), 128.2(\mathrm{CH}), 125.4(\mathrm{CH}), 72.8(\mathrm{CH}), 50.4$ $\left(\mathrm{CH}_{2}\right)$. $\left(\mathrm{ESI}^{+}, \mathrm{m} / \mathrm{z}\right)$ : calcd for $\left(\mathrm{C}_{11} \mathrm{H}_{11} \mathrm{FNaO}\right)^{+}(\mathrm{M}+\mathrm{Na})^{+}$: 201.0686; found 201.0690.

2-Chloro-1-(2-chlorophenyl)ethan-1-ol (3j): Colorless oil. $R_{\mathrm{f}}(20 \%$ EtOAc/hexane): 0.50. IR: v 3369, 3005, 2987, 1267, 1260 and $761 \mathrm{~cm}^{-1} .{ }^{1} \mathrm{H}-\mathrm{NMR}\left(300.13 \mathrm{MHz}, \mathrm{CDCl}_{3}\right)$ : $\delta 7.64(d d, J=9.1,1.8 \mathrm{~Hz}, 1 \mathrm{H}), 7.38-7.24(m, 3 \mathrm{H}), 5.31(d t, J=8.9,2.9 \mathrm{~Hz}, 1 \mathrm{H}), 3.91$ $(d d, J=11.3,2.9 \mathrm{~Hz}, 1 \mathrm{H}), 3.55(d d, J=11.3,8.7 \mathrm{~Hz}, 1 \mathrm{H}), 2.75(d, J=3.4 \mathrm{~Hz}, 1 \mathrm{H}) .{ }^{13} \mathrm{C}-$ NMR (75.5 MHz, $\left.\mathrm{CDCl}_{3}\right): \delta 137.2(\mathrm{C}), 131.9(\mathrm{C}), 129.5(\mathrm{CH}), 129.4(\mathrm{CH}), 127.5(\mathrm{CH})$, $127.2(\mathrm{CH}), 70.7(\mathrm{CH}), 49.5\left(\mathrm{CH}_{2}\right)$. $\left(\mathrm{ESI}^{+}, \mathrm{m} / \mathrm{z}\right)$ : calcd for $\left(\mathrm{C}_{8} \mathrm{H}_{8} \mathrm{Cl}_{2} \mathrm{NaO}\right)^{+}(\mathrm{M}+\mathrm{Na})^{+}$: 212.9844; found 212.9844 .

1-Chlorooctan-2-ol (3k): Yellowish oil. $R_{\mathrm{f}}(10 \%$ EtOAc/hexane): 0.37 . IR: $v$ 3375, 3005, 2987, 1275, 1260 and $763 \mathrm{~cm}^{-1} .{ }^{1} \mathrm{H}-\mathrm{NMR}\left(300.13 \mathrm{MHz}, \mathrm{CDCl}_{3}\right): \delta 3.79(d t t, J=$ 7.0, 5.3, $2.7 \mathrm{~Hz}, 1 \mathrm{H}), 3.63(d d, J=11.0,3.2 \mathrm{~Hz}, 1 \mathrm{H}), 3.47(d d, J=11.1,7.1 \mathrm{~Hz}, 1 \mathrm{H})$, $2.22(b r s, 1 \mathrm{H}), 1.57-1.47(m, 2 \mathrm{H}), 1.30(m, 8 \mathrm{H}), 0.88(m, 3 \mathrm{H}) .{ }^{13} \mathrm{C}-\mathrm{NMR}(75.5 \mathrm{MHz}$, $\left.\mathrm{CDCl}_{3}\right): \delta 71.5(\mathrm{CH}), 50.6\left(\mathrm{CH}_{2}\right), 34.2\left(\mathrm{CH}_{2}\right), 31.7\left(\mathrm{CH}_{2}\right), 29.2\left(\mathrm{CH}_{2}\right), 25.5\left(\mathrm{CH}_{2}\right), 22.6$ 
$\left(\mathrm{CH}_{2}\right), 14.1\left(\mathrm{CH}_{3}\right)$. $\left(\mathrm{ESI}^{+}, \mathrm{m} / \mathrm{z}\right)$ : calcd for $\left(\mathrm{C}_{11} \mathrm{H}_{11} \mathrm{FNaO}\right)^{+}(\mathrm{M}+\mathrm{Na})^{+}$: 201.0686; found 201.0690.

2-Bromo-1-phenylethan-1-ol (3I): Colorless oil. $R_{\mathrm{f}}(20 \%$ EtOAc/hexane): 0.43 . IR: $v$ 3401, 3005, 2988, 1267, 1260 and $764 \mathrm{~cm}^{-1} .{ }^{1} \mathrm{H}-\mathrm{NMR}\left(300.13 \mathrm{MHz}, \mathrm{CDCl}_{3}\right): \delta 7.40-7.33$ $(m, 5 \mathrm{H}), 4.93(d t, J=9.0,3.3 \mathrm{~Hz}, 1 \mathrm{H}), 3.65(d d, J=10.4,3.4 \mathrm{~Hz}, 1 \mathrm{H}), 3.55(d d, J=10.4$, $8.9 \mathrm{~Hz}, 1 \mathrm{H}), 2.67(d, J=3.3 \mathrm{~Hz}, 1 \mathrm{H}) .{ }^{13} \mathrm{C}-\mathrm{NMR}\left(75.5 \mathrm{MHz}, \mathrm{CDCl}_{3}\right): \delta 140.3(\mathrm{C}), 128.7$ $(2 \mathrm{CH}), 128.5(\mathrm{C}), 126.0(2 \mathrm{CH}), 73.8(\mathrm{CH}), 40.3\left(\mathrm{CH}_{2}\right) .\left(\mathrm{ESI}^{+}, \mathrm{m} / \mathrm{z}\right)$ : calcd for $\left(\mathrm{C}_{8} \mathrm{H}_{9} \mathrm{BrNaO}\right)^{+}(\mathrm{M}+\mathrm{Na})^{+}: 222.9729$; found 222.9731 .

\section{Scale-up of the one-pot cascade hydration-bioreduction process of alkyne 1a}

Table S11. Scale-ups of the one-pot cascade hydration-bioreduction processes of alkyne 1a.

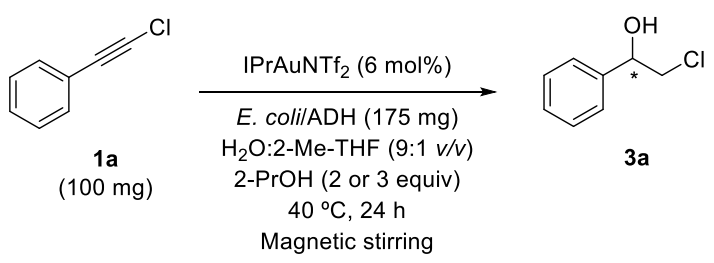

\begin{tabular}{lcccccc}
\hline Entry & Enzyme & $\mathbf{1 a}(\%)^{\mathrm{a}}$ & $\mathbf{2 a}(\%)^{\mathrm{a}}$ & $\begin{array}{c}\text { By-products } \\
(\%)^{\mathrm{a}}\end{array}$ & $\mathbf{3 a}(\%)^{\mathrm{a}}$ & $\mathbf{3 a} e e(\%)^{\mathrm{b}}$ \\
\hline 1 & LbADH & $<1$ & $<1$ & 5 & $95(82)$ & $>99(S)$ \\
2 & ADH-A & $<1$ & $<1$ & 6 & $94(83)$ & $99(R)$ \\
\hline
\end{tabular}




\section{Chemical epoxide formation from 2-chloro-1-phenylethan-1-ol (3a)}

Table S12. Optimisation of the epoxide formation process from alcohol 3a in terms of base amount.

\begin{tabular}{|c|c|c|c|}
\hline & $\begin{array}{c}r a c-3 a \\
(100 \mathrm{mM})\end{array}$ & $\begin{array}{c}\mathrm{NaOH} \text { (x equiv) } \\
\mathrm{H}_{2} \mathrm{O}: 2-\mathrm{Me}-\mathrm{THF}(9: 1 \mathrm{v/v}) \\
2-\mathrm{PrOH}(2 \text { equiv) } \\
40^{\circ} \mathrm{C}, 3 \mathrm{~h} \\
\text { Magnetic stiring }\end{array}$ & \\
\hline Entry & $\mathrm{NaOH}$ (equiv) & $3 \mathbf{a}(\%)^{\mathrm{a}}$ & Epoxide $4 \mathbf{a}(\%)^{2}$ \\
\hline 1 & 0.3 & 80 & 20 \\
\hline 2 & 0.4 & 71 & 29 \\
\hline 3 & 0.5 & 59 & 41 \\
\hline 4 & 0.6 & 48 & 52 \\
\hline 5 & 1.0 & 16 & 84 \\
\hline 6 & 1.2 & 1 & 99 \\
\hline 7 & 1.3 & 1 & 99 \\
\hline 8 & 1.4 & 1 & 99 \\
\hline
\end{tabular}

${ }^{\text {a }}$ Product percentages were determined by GC analysis using calibration curves.

XIII. Development of a hydration-bioreduction-epoxide formation sequence to obtain both optically active epoxide 4 a enantiomers

To obtain the desired $\mathbf{4 a}$ enantiomer, the previously described hydration-bioreduction protocol was followed using either $\mathrm{LbADH}$ or $\mathrm{ADH}-\mathrm{A}$. Then, $\mathrm{NaOH}$ (1.2 equiv as a $\mathrm{NaOH} 3 \mathrm{M}$ aqueous solution) was added, and the mixture kept under magnetic stirring at $40{ }^{\circ} \mathrm{C}$ for $3 \mathrm{~h}$. After this time, the solution was extracted with EtOAc $(5 \times 1 \mathrm{~mL})$, the organic layers combined, dried over anhydrous $\mathrm{Na}_{2} \mathrm{SO}_{4}$ and filtered. The solvent was evaporated under reduced pressure, measuring then the conversions into the styrene oxide 4a by GC analysis and the enantiomeric excess by HPLC. Styrene oxide 4a was purified by column chromatography on silica gel (88-92 isolated yield, see Table S13). 
Table S13. Sequence transformation involving an alkyne hydration-bioreduction-epoxide formation.
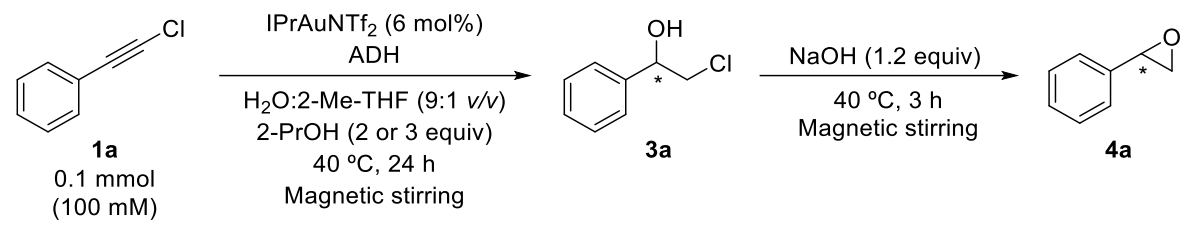

\begin{tabular}{cccccccc}
\hline Entry & Enzyme & $\mathbf{1 a}(\%)^{\mathrm{a}}$ & $\mathbf{2 a}(\%)^{\mathrm{a}}$ & ${\text { By-products }(\%)^{\mathrm{a}}}^{\mathbf{3 a}(\%)^{\mathrm{a}}}$ & $\mathbf{4 a}(\%)^{\mathrm{a}}$ & $\mathbf{4 a} e e(\%)^{\mathrm{b}}$ \\
\hline 1 & LbADH & $<1$ & $<1$ & $<1$ & 2 & $98(92)$ & $>99(S)$ \\
2 & ADH-A & $<1$ & $<1$ & $<1$ & 4 & $96(88)$ & $97(R)$ \\
\hline
\end{tabular}

a Product percentages were determined by GC analysis using calibration curves.

${ }^{\mathrm{b}}$ Enantiomeric excess values were measured by HPLC. Absolute configurations of epoxide 4a were assigned by comparison of the retention times with the one obtained for commercially available $(R)-\mathbf{4 a}$.

Styrene oxide (4a): Colorless oil. $R_{\mathrm{f}}\left(20 \%\right.$ EtOAc/hexane): $0.65 .{ }^{1} \mathrm{H}-\mathrm{NMR}(300.13 \mathrm{MHz}$, $\left.\mathrm{CDCl}_{3}\right): \delta 7.40-7.27(m, 5 \mathrm{H}), 3.87(d d, J=4.1,2.6 \mathrm{~Hz}, 1 \mathrm{H}), 3.16(d d, J=5.4,4.1 \mathrm{~Hz}$, $1 \mathrm{H}), 2.81(d d, J=5.5 .2 .6 \mathrm{~Hz}, 1 \mathrm{H}) .{ }^{13} \mathrm{C}-\mathrm{NMR}\left(75.5 \mathrm{MHz}, \mathrm{CDCl}_{3}\right): \delta 137.6(\mathrm{C}), 128.5$ $(2 \mathrm{CH}), 128.2(\mathrm{C}), 125.5(2 \mathrm{CH}), 52.4(\mathrm{CH}), 51.2\left(\mathrm{CH}_{2}\right) .\left(\mathrm{ESI}^{+}, \mathrm{m} / \mathrm{z}\right)$ : calcd for $\left(\mathrm{C}_{8} \mathrm{H}_{9} \mathrm{O}\right)^{+}$ $(\mathrm{M}+\mathrm{H})^{+}:$121.0648; found 121.0648 .

\section{Analytical data}

\section{XIV.1. GC analyses for the determination of product percentages}

An Agilent DB-1701 (30 m x 0.25 cm x $0.25 \mu \mathrm{m}, 12.2 \mathrm{psi} \mathrm{N}$ ), HP-1 (30 m x $0.32 \mathrm{~mm}$ x $0.25 \mu \mathrm{m}, 12.2 \mathrm{psi}_{2}$ ) or a HP-5 (30 $\mathrm{m} \times 0.32 \mathrm{~mm} \times 0.25 \mu \mathrm{m}, 12.2 \mathrm{psi} \mathrm{N}_{2}$ ) column were used for the determination of the conversion values in the cascade and sequential protocols. The experimental conditions are indicated in Table S14. 
Table S14. GC analytical conditions and retention times for the determination of conversion values.

\begin{tabular}{|c|c|c|c|c|}
\hline Entry & Substrate & Column & Program $^{a}$ & Retention time (min) \\
\hline 1 & 1a & HP-1 & 90/0/1/110/2/30/200/1 & 3.1 \\
\hline 2 & $2 a$ & HP-1 & $90 / 0 / 1 / 110 / 2 / 30 / 200 / 1$ & 7.4 \\
\hline 3 & 3a & HP-1 & $90 / 0 / 1 / 110 / 2 / 30 / 200 / 1$ & 7.2 \\
\hline 4 & $\mathbf{1 b}$ & HP-1 & $90 / 0 / 1 / 110 / 2 / 30 / 200 / 1$ & 3.0 \\
\hline 5 & $2 b$ & HP-1 & $90 / 0 / 1 / 110 / 2 / 30 / 200 / 1$ & 6.8 \\
\hline 6 & $3 \mathbf{b}$ & HP-1 & $90 / 0 / 1 / 110 / 2 / 30 / 200 / 1$ & 7.2 \\
\hline 7 & $1 \mathrm{c}$ & HP-5 & $90 / 0 / 2 / 130 / 0 / 20 / 200 / 1$ & 7.1 \\
\hline 8 & $2 c$ & HP-5 & 90/0/2/130/0/20/200/1 & 15.2 \\
\hline 9 & $3 c$ & HP-5 & $90 / 0 / 2 / 130 / 0 / 20 / 200 / 1$ & 15.9 \\
\hline 10 & 1d & HP-5 & $90 / 0 / 2 / 130 / 0 / 20 / 200 / 1$ & 10.2 \\
\hline 11 & 2d & HP-5 & $90 / 0 / 2 / 130 / 0 / 20 / 200 / 1$ & 19.8 \\
\hline 12 & 3d & HP-5 & $90 / 0 / 2 / 130 / 0 / 20 / 200 / 1$ & 20.6 \\
\hline 13 & $1 \mathrm{e}$ & $\mathrm{HP}-1$ & $90 / 0 / 2 / 130 / 0 / 20 / 200 / 1$ & 4.9 \\
\hline 14 & $2 e$ & HP-1 & $90 / 0 / 2 / 130 / 0 / 20 / 200 / 1$ & 10.8 \\
\hline 15 & $3 e$ & HP-1 & $90 / 0 / 2 / 130 / 0 / 20 / 200 / 1$ & 9.9 \\
\hline 16 & 1f & $\mathrm{HP}-5$ & $90 / 0 / 2 / 130 / 0 / 20 / 200 / 1$ & 10.8 \\
\hline 17 & $2 f$ & HP-5 & $90 / 0 / 2 / 130 / 0 / 20 / 200 / 1$ & 20.8 \\
\hline 18 & 3f & HP-5 & $90 / 0 / 2 / 130 / 0 / 20 / 200 / 1$ & 19.0 \\
\hline 19 & $1 \mathrm{~g}$ & Chiralsil Dex CB & $110 / 0 / 2.5 / 120 / 0 / 10 / 200 / 2$ & 7.0 \\
\hline 20 & $2 \mathrm{~g}$ & Chiralsil Dex CB & $110 / 0 / 2.5 / 120 / 0 / 10 / 200 / 2$ & 11.0 \\
\hline 21 & $3 g$ & Chiralsil Dex CB & $110 / 0 / 2.5 / 120 / 0 / 10 / 200 / 2$ & 12.5 \\
\hline 22 & $1 \mathrm{~h}$ & Chiralsil Dex CB & $110 / 0 / 2.5 / 120 / 0 / 10 / 200 / 2$ & 8.2 \\
\hline 23 & $2 \mathrm{~h}$ & Chiralsil Dex CB & $110 / 0 / 2.5 / 120 / 0 / 10 / 200 / 2$ & 11.6 \\
\hline 24 & $3 \mathbf{h}$ & Chiralsil Dex CB & $110 / 0 / 2.5 / 120 / 0 / 10 / 200 / 2$ & 12.6 \\
\hline 25 & $\mathbf{1 i}$ & $\mathrm{HP}-1$ & $90 / 0 / 1 / 110 / 2 / 30 / 200 / 1$ & 17.1 \\
\hline 26 & $2 \mathbf{i}$ & HP-1 & $90 / 0 / 1 / 110 / 2 / 30 / 200 / 1$ & 24.2 \\
\hline 27 & $3 \mathbf{i}$ & HP-1 & $90 / 0 / 1 / 110 / 2 / 30 / 200 / 1$ & 25.3 \\
\hline 28 & $\mathbf{1 j}$ & $\mathrm{HP}-1$ & $90 / 0 / 1 / 110 / 2 / 30 / 200 / 1$ & 8.1 \\
\hline 29 & $2 \mathbf{j}$ & HP-1 & $90 / 0 / 1 / 110 / 2 / 30 / 200 / 1$ & 13.6 \\
\hline 30 & $\mathbf{3 j}$ & HP-1 & $90 / 0 / 1 / 110 / 2 / 30 / 200 / 1$ & 15.2 \\
\hline 31 & $1 \mathrm{k}$ & $\mathrm{HP}-1$ & $70 / 0 / 2 / 120 / 2 / 200 / 1$ & 3.9 \\
\hline 32 & $2 \mathbf{k}$ & HP-1 & $70 / 0 / 2 / 120 / 2 / 200 / 1$ & 8.0 \\
\hline 33 & 3k & HP-1 & $70 / 0 / 2 / 120 / 2 / 200 / 1$ & 9.4 \\
\hline 34 & 11 & HP-5 & $90 / 0 / 2 / 130 / 0 / 20 / 200 / 1$ & 5.3 \\
\hline 35 & 21 & HP-5 & $90 / 0 / 2 / 130 / 0 / 20 / 200 / 1$ & 11.1 \\
\hline 36 & 31 & HP-5 & $90 / 0 / 2 / 130 / 0 / 20 / 200 / 1$ & 10.8 \\
\hline 37 & $4 \mathbf{a}$ & HP-1 & $90 / 0 / 1 / 110 / 2 / 30 / 200 / 1$ & 2.9 \\
\hline
\end{tabular}




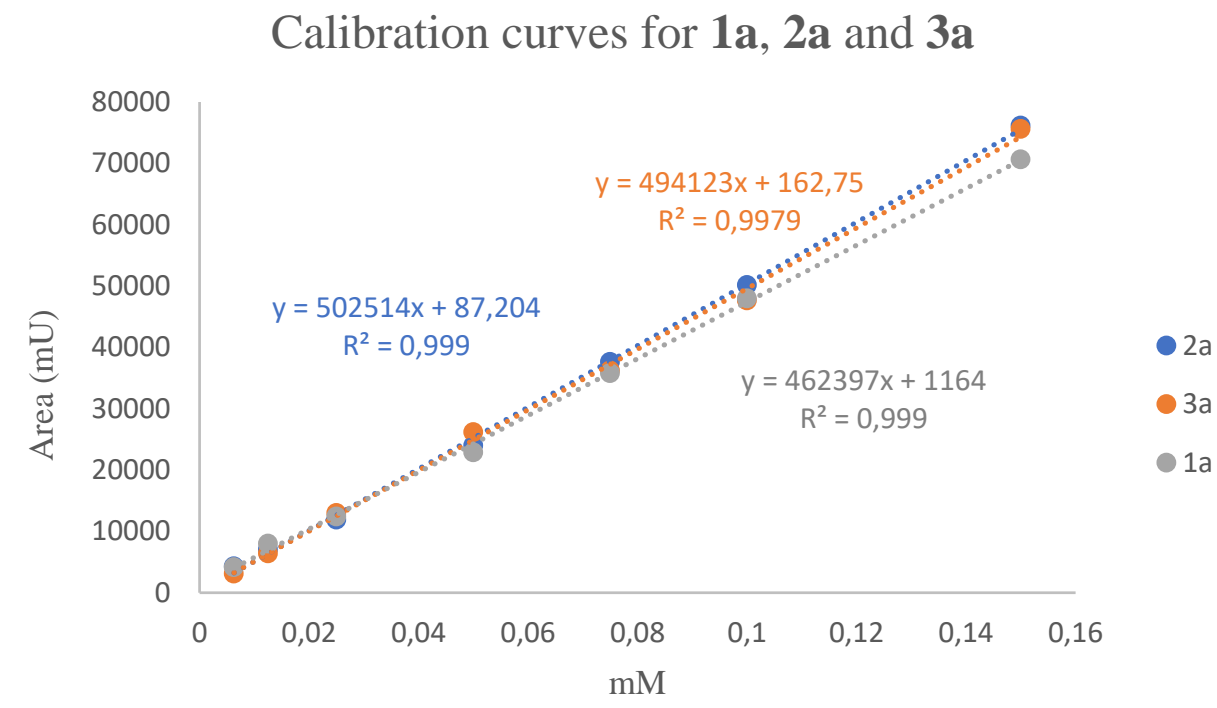

Figure S5. Calibration curves for compounds 1a, 2a and 3a.



Figure S6. Calibration curves for compounds $\mathbf{1 b}, \mathbf{2 b}$ and $\mathbf{3 b}$. 


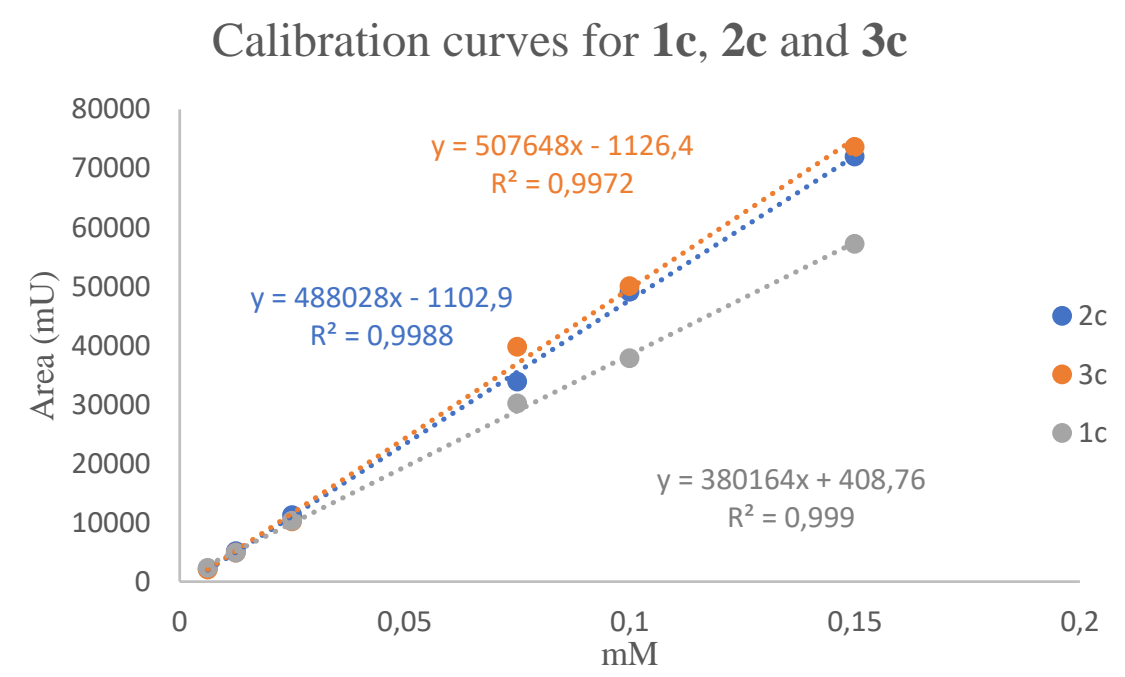

Figure S7. Calibration curves for compounds $1 \mathbf{c}, \mathbf{2 c}$ and $\mathbf{3 c}$.



Figure S8. Calibration curves for compounds $\mathbf{1 d}$, 2d and $\mathbf{3 d}$.

\section{Calibration curves for $\mathbf{1 e}, \mathbf{2 e}$ and $\mathbf{3 e}$}

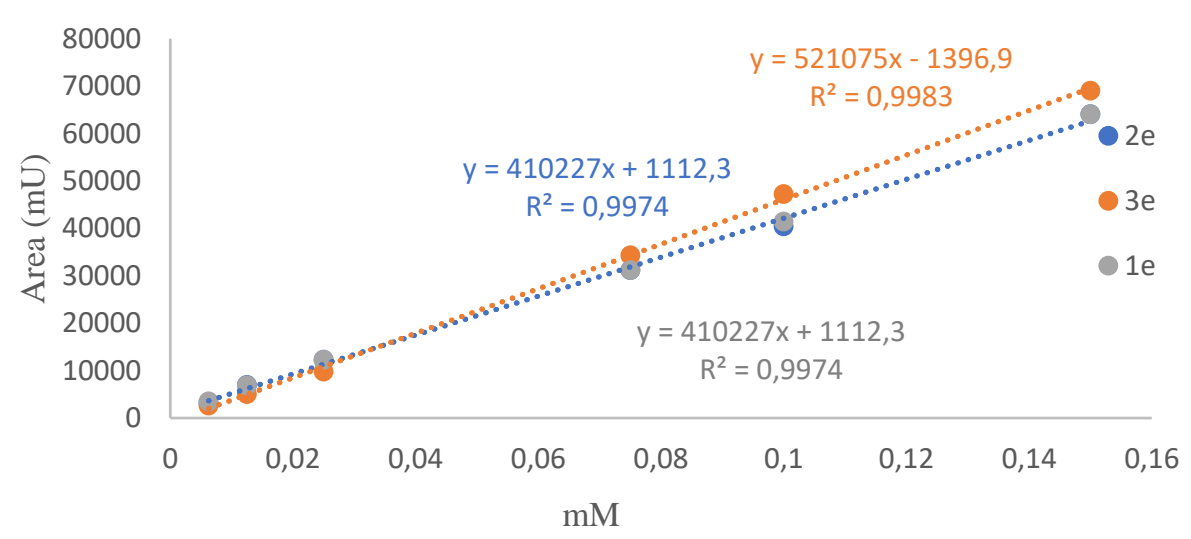

Figure S9. Calibration curves for compounds 1e, 2e and 3e. 


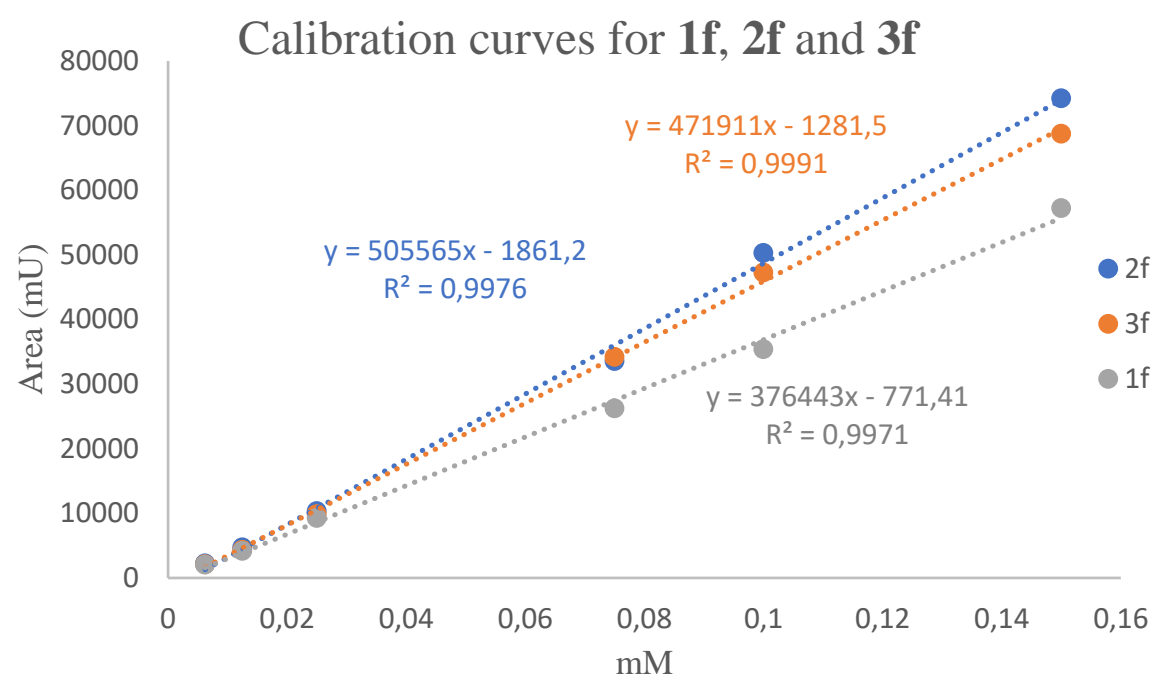

Figure S10. Calibration curves for compounds $1 \mathbf{f}, \mathbf{2 f}$ and $\mathbf{3 f}$.



Figure S11. Calibration curves for compounds 1g, 2g and 3g.

Calibration curves for $\mathbf{1 h}, \mathbf{2 h}$ and $\mathbf{3 h}$

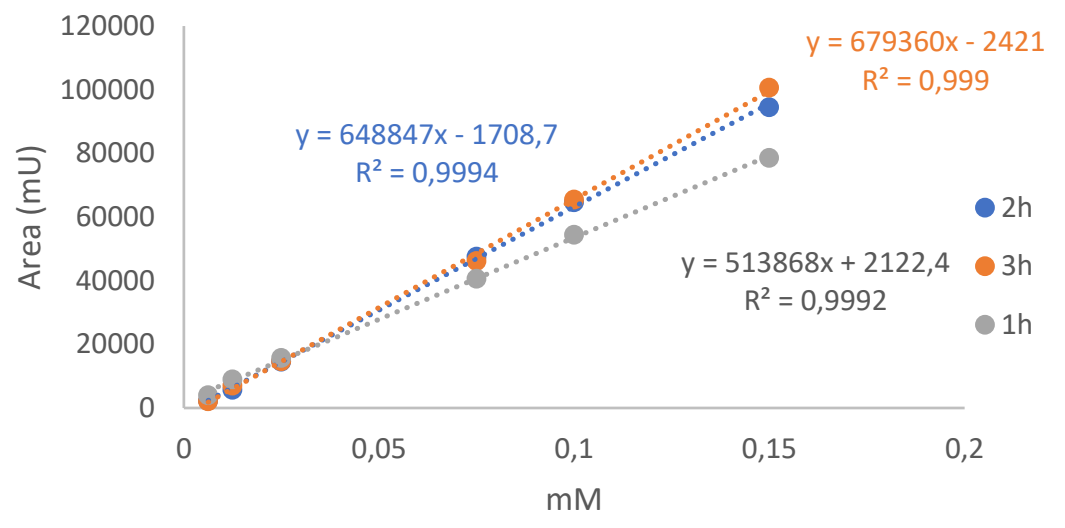

Figure S12. Calibration curves for compounds $1 \mathbf{h}, \mathbf{2 h}$ and $\mathbf{3 h}$. 


\section{Calibration curves for $\mathbf{1} \mathbf{i}, \mathbf{2} \mathbf{i}$ and $\mathbf{3 i}$}

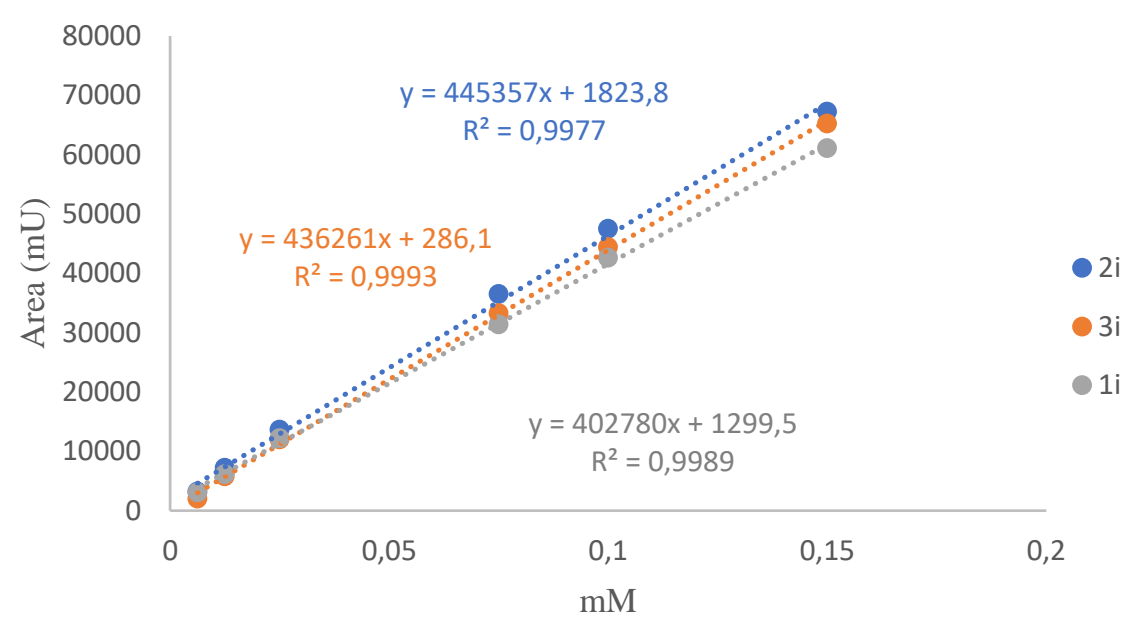

Figure S13. Calibration curves for compounds 1i, $2 \mathbf{i}$ and $\mathbf{3 i}$.

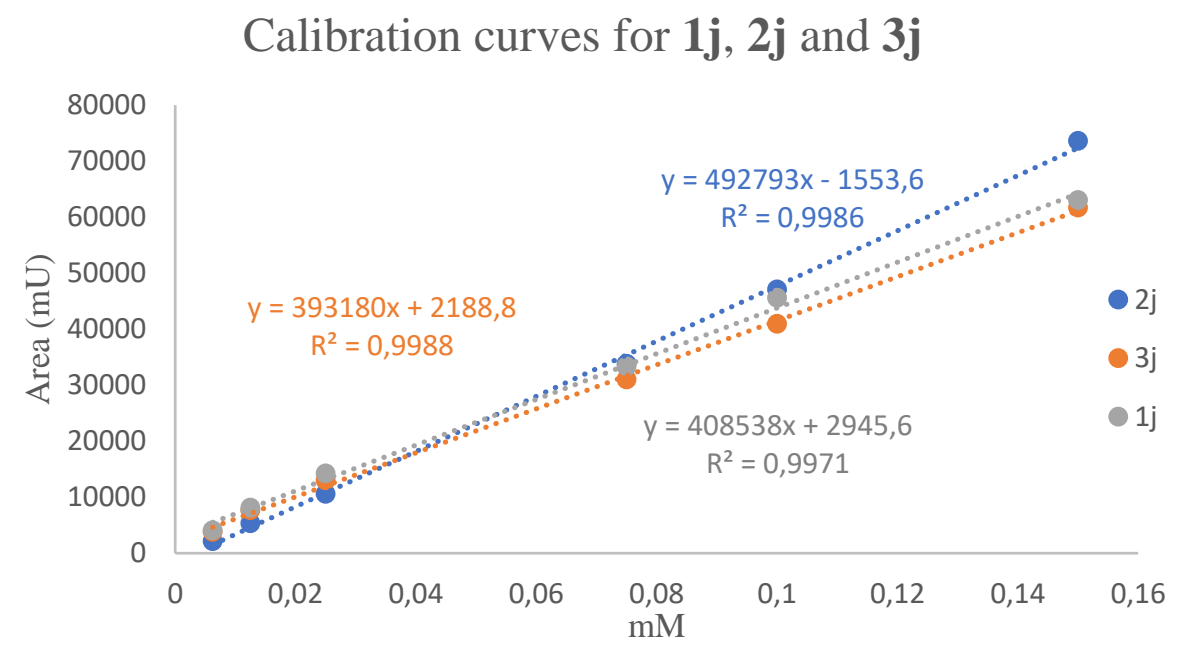

Figure S14. Calibration curves for compounds $\mathbf{1} \mathbf{j}, \mathbf{2} \mathbf{j}$ and $\mathbf{3 j}$.

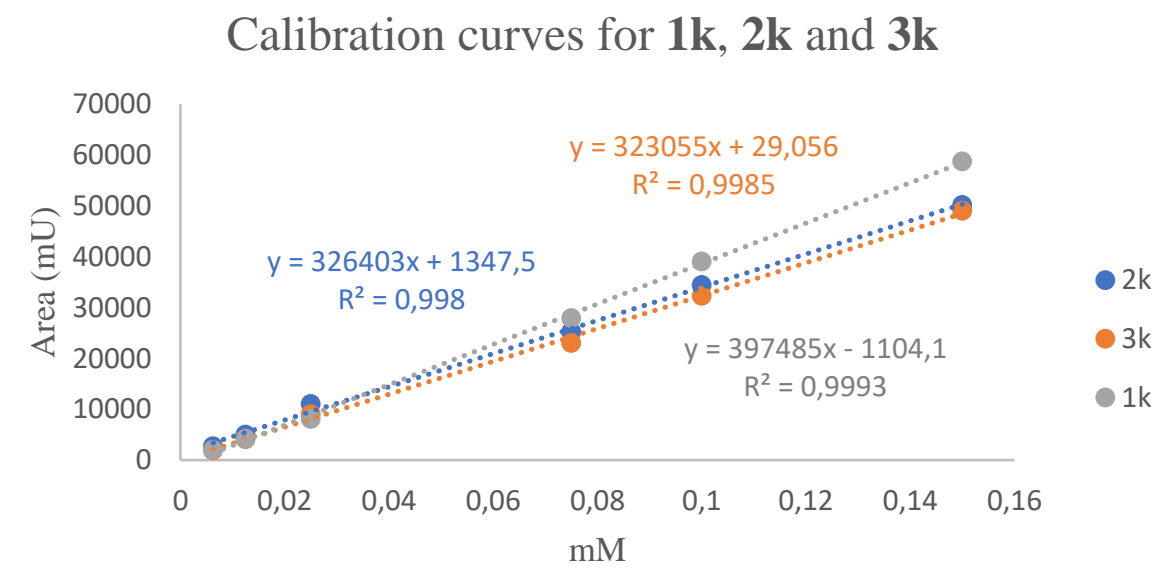

Figure S15. Calibration curves for compounds $1 \mathbf{k}, \mathbf{2 k}$ and $\mathbf{3 k}$. 
Calibration curves for $\mathbf{1 1}, \mathbf{2 l}$ and $\mathbf{3 1}$

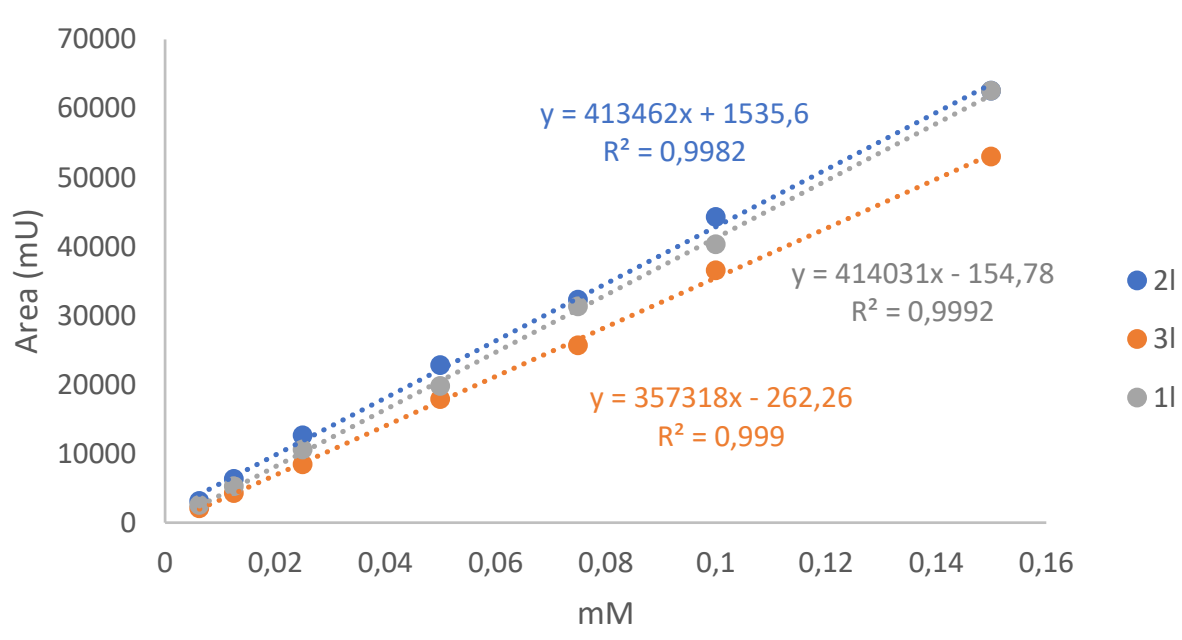

Figure S16. Calibration curves for compounds $\mathbf{1 1}, \mathbf{2 l}$ and $\mathbf{3 1}$.

\section{Styrene oxide (4a)}

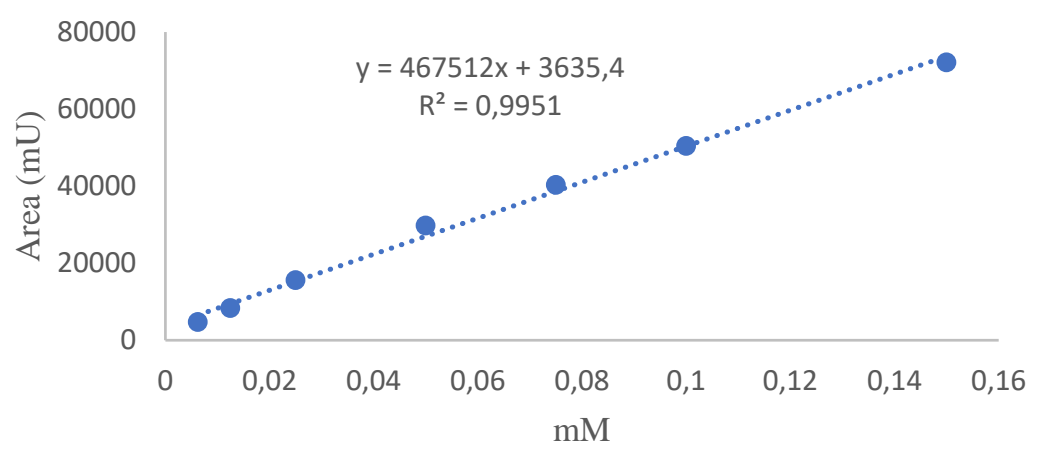

Figure S17. Calibration curves for styrene oxide (4a). 
XIV.3. GC analyses for the determination of ee values of alcohols $3 g, h, k$

Chiralsil Dex CB (30 m x $0.32 \mathrm{~m} \times 0.25 \mu \mathrm{m}, 12.2 \mathrm{psi} \mathrm{N}_{2}$ ) was employed for the determination of the enantiomeric excess values of alcohols $\mathbf{3 g}, \mathbf{h}, \mathbf{k}$ (Table S15).

Table S15. GC analyses for the determination of the $e e$ values of alcohols $\mathbf{3 g}, \mathbf{h}, \mathbf{k}$.

\begin{tabular}{|c|c|c|c|c|}
\hline Entry & Alcohol & Program $^{a}$ & \multicolumn{2}{|c|}{ Retention time (min) ${ }^{b}$} \\
\hline 1 & $3 g$ & $110 / 0 / 2.5 / 120 / 0 / 10 / 200 / 5$ & $12.0(R)$ & $12.2(S)$ \\
\hline 2 & $3 \mathbf{h}$ & $110 / 0 / 2.5 / 120 / 0 / 10 / 200 / 5$ & $12.7(R)$ & $12.9(S)$ \\
\hline 3 & 3k & $60 / 1 / 10 / 110 / 0 / 0.5 / 124 / 0 / 10 / 180 / 1$ & $18.1(R)$ & $19.9(S)$ \\
\hline
\end{tabular}



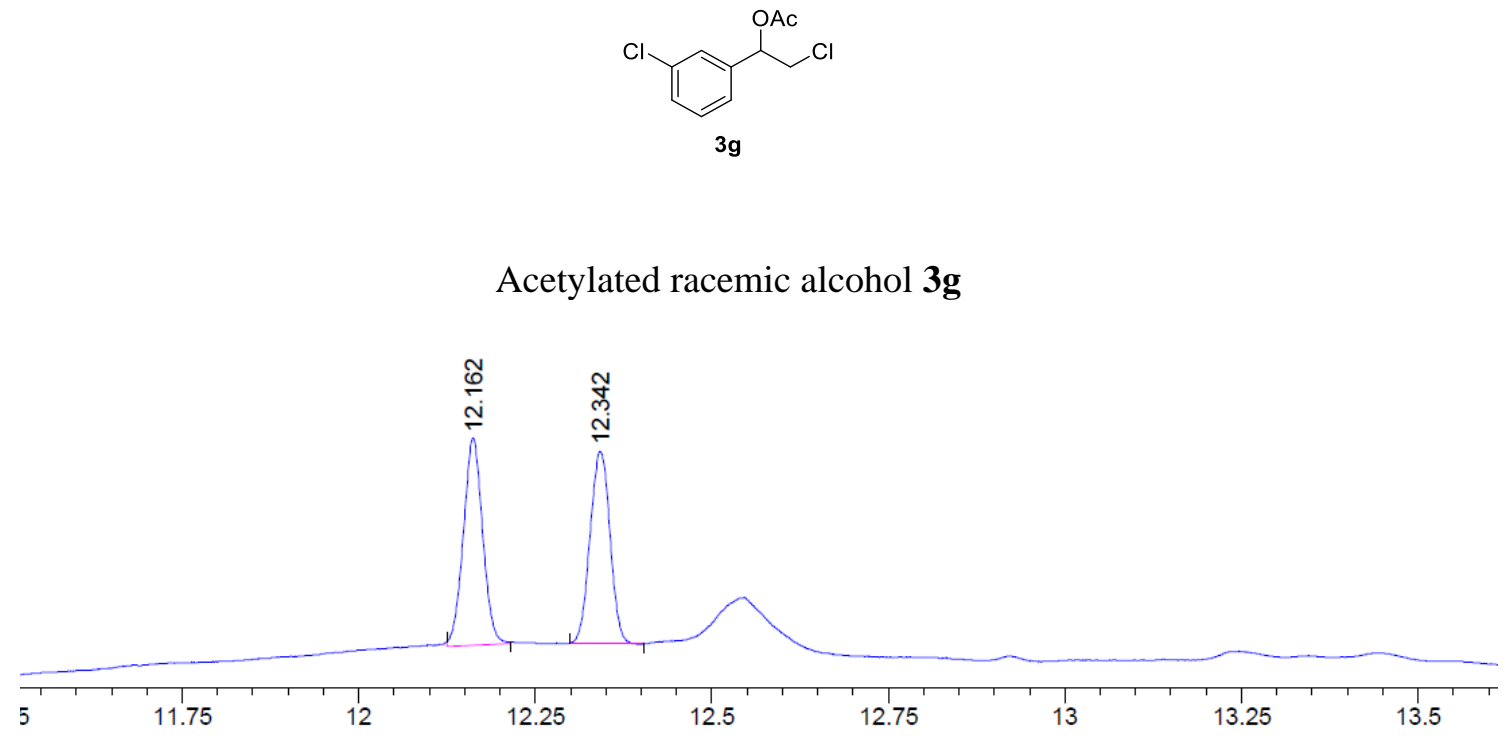

Acetylated alcohol (S)-3g in $>99 \%$ ee (after bioreduction with $L b \mathrm{ADH}$ )

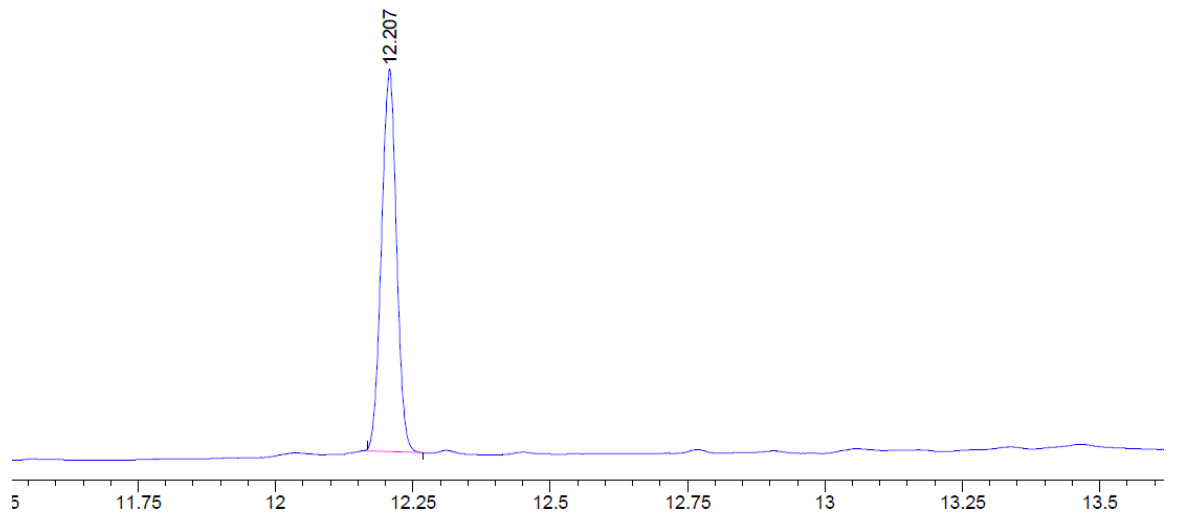

Acetylated alcohol $(R)-\mathbf{3 g}$ in $>99 \%$ ee (after bioreduction with ADH-A)

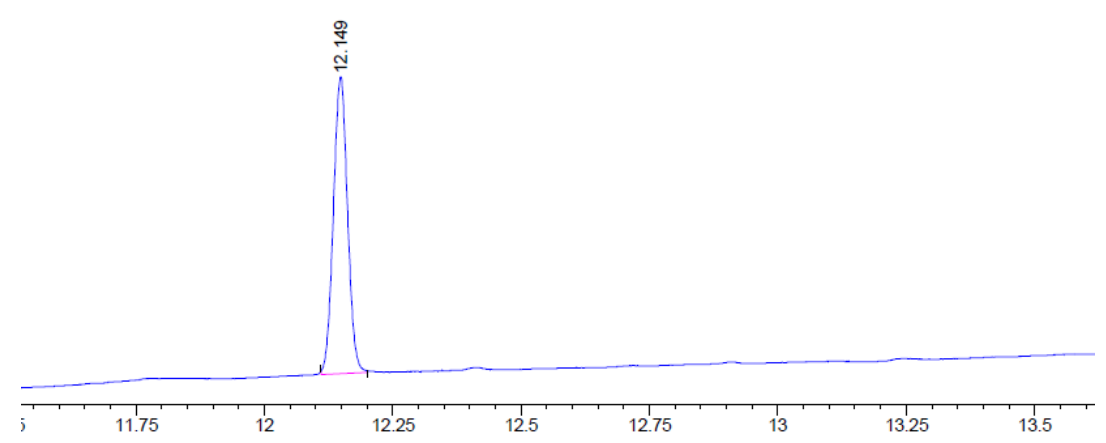

Figure S18. GC chromatograms of acetylated racemic halohydrin and optically active $\mathbf{3 g}$ obtained using selective ADHs. 


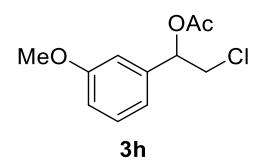

Acetylated racemic alcohol 3h



Acetylated alcohol (S)-3h in $>99 \%$ ee (after bioreduction with $L b \mathrm{ADH}$ )

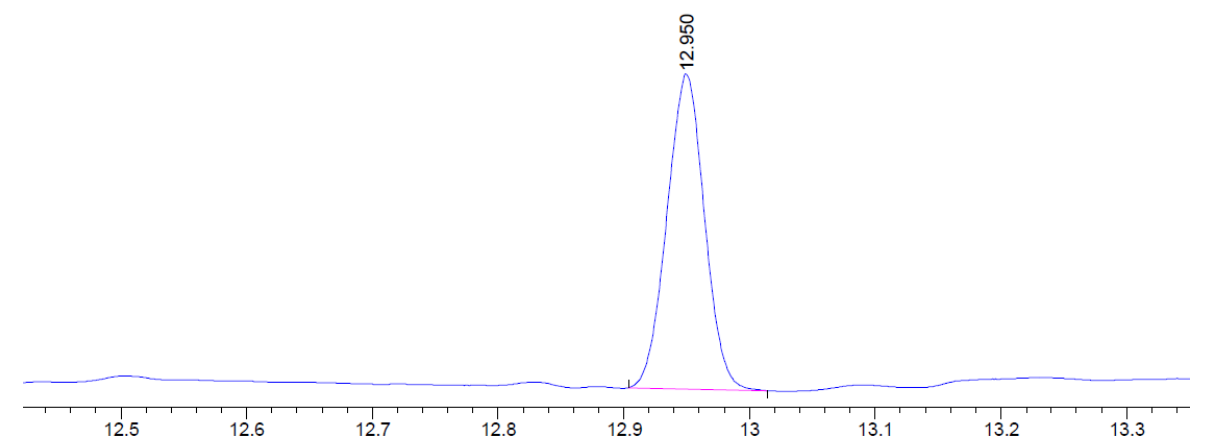

Acetylated alcohol $(R)-3 \mathbf{h}$ in $>99 \%$ ee (after bioreduction with ADH-A)

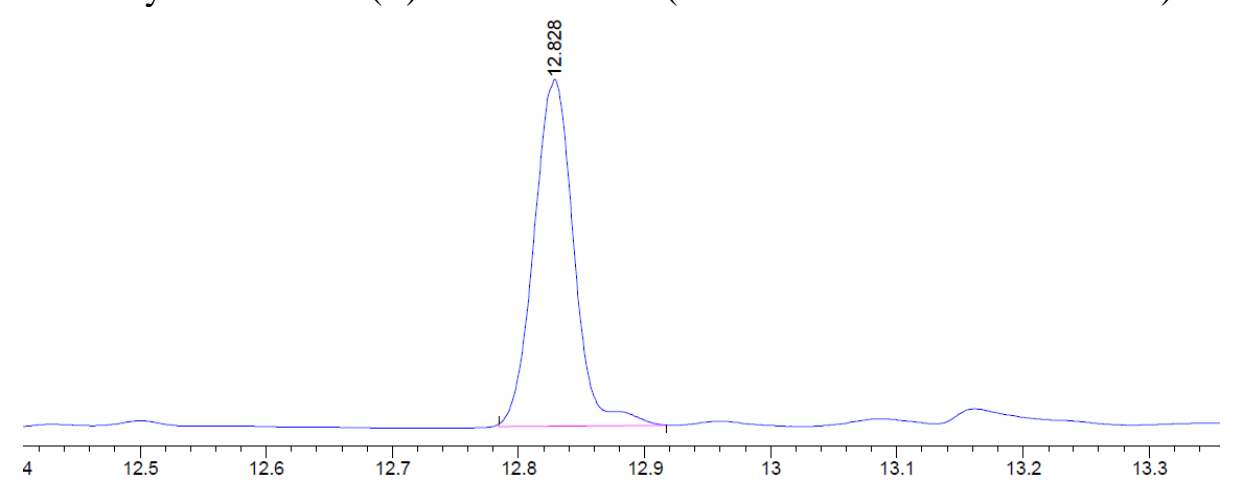

Figure S19. GC chromatograms of acetylated racemic halohydrin and optically active $\mathbf{3 h}$ obtained using selective ADHs. 




3k

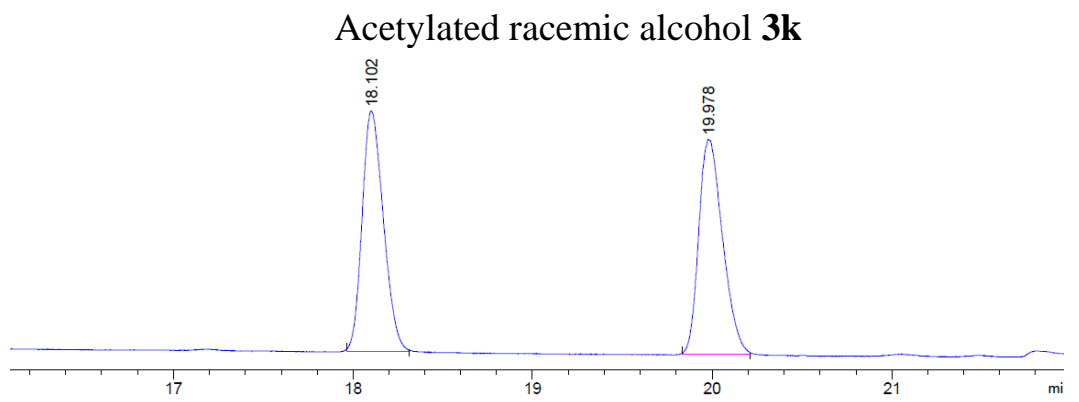

Acetylated alcohol (S)-3k in $>99 \%$ ee (after bioreduction with $L b \mathrm{ADH})$

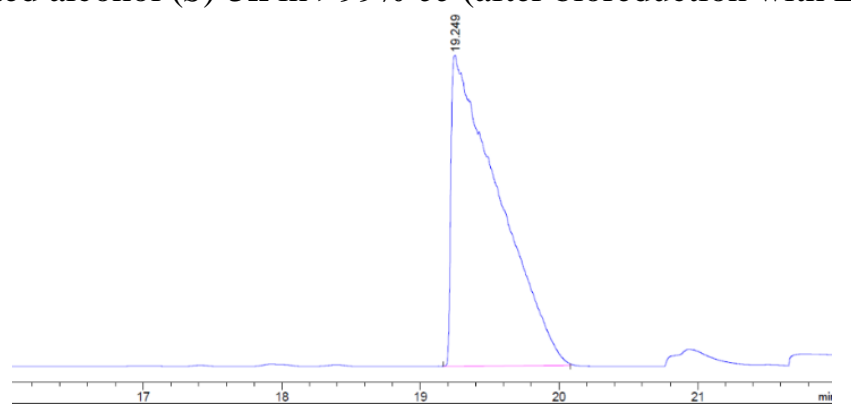

Acetylated alcohol $(R)-3 \mathbf{k}$ in $>99 \%$ ee (after bioreduction with ADH-A)

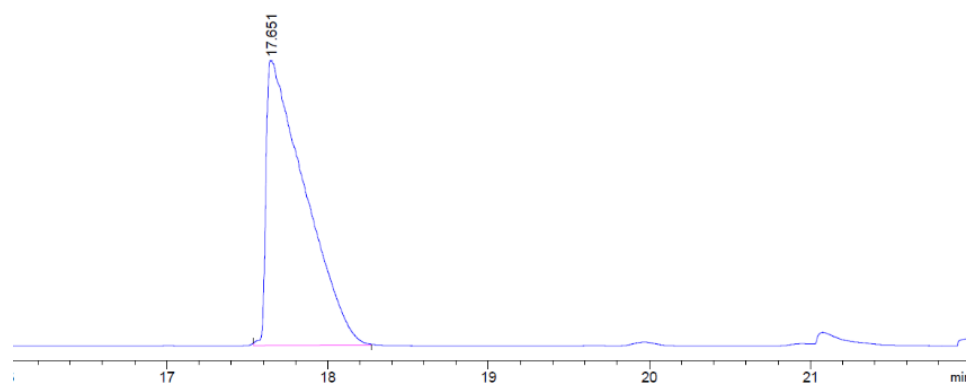

Acetylated alcohol $(R)-3 \mathbf{k}$ in $>99 \%$ ee (after bioreduction with ADH-T)

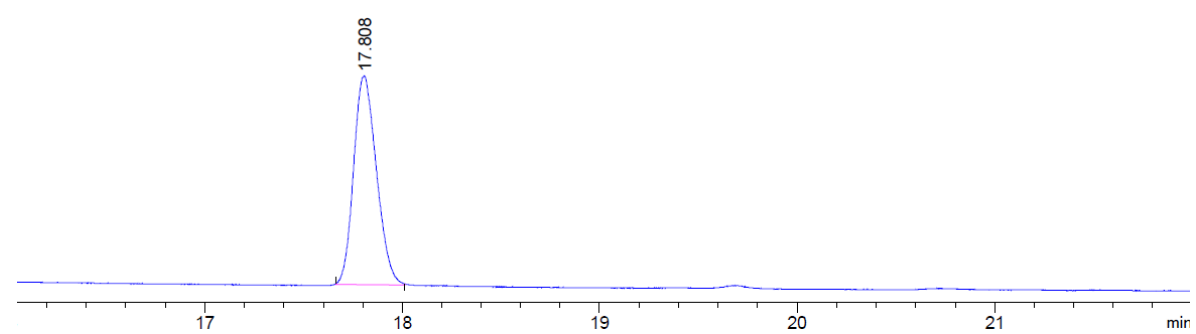

Figure S20. GC chromatograms of acetylated racemic halohydrin and optically active 3k obtained using selective ADHs. 
XIV.4. HPLC analyses for the determination of enantiomeric excess values of alcohols 3a-f,i,j,l and epoxide $4 a$

Enantiomeric excess (ee) values of halohydrins $\mathbf{3 a - f}, \mathbf{i}, \mathbf{j}, \mathbf{l}$ and styrene oxide (4a) were determined by HPLC analyses, using different columns and conditions as specified in Section I and in Table S16.

Table S16. HPLC analytical conditions and retention times for the determination of enantiomeric excess values of alcohols $3 \mathbf{a}-\mathbf{f}, \mathbf{i}, \mathbf{j}, \mathbf{l}$ and epoxide $\mathbf{4 a} .^{a}$

\begin{tabular}{cccccc}
\hline Entry & Substrate & Column & $\begin{array}{c}n \text {-Hexane/2-propanol } \\
(v / v)\end{array}$ & $\begin{array}{c}(R) \text {-alcohol } \\
(\mathrm{min})\end{array}$ & $\begin{array}{c}(S) \text {-alcohol } \\
(\mathrm{min})\end{array}$ \\
\hline 1 & $\mathbf{3 a}$ & Chiralcel OJ-H & $95: 5$ & 25.9 & 28.2 \\
2 & $\mathbf{3 b}$ & Chiralpak AD-H & $95: 5$ & 14.2 & 15.1 \\
3 & $\mathbf{3 c}$ & Chiralcel OJ-H & $95: 5$ & 21.0 & 22.7 \\
4 & $\mathbf{3 d}$ & Chiralpak AD-H & $95: 5$ & 17.0 & 17.9 \\
5 & $\mathbf{3 e}$ & Chiralpak IA & $95: 5$ & 12.4 & 11.7 \\
6 & $\mathbf{3 f}$ & Chiralpak IA & $95: 5$ & 18.3 & 17.0 \\
7 & $\mathbf{3 i}$ & Chiralcel OJ-H & $95: 5$ & 18.8 & 20.6 \\
8 & $\mathbf{3 j}$ & Chiralpak IA & $95: 5$ & 11.6 & 10.9 \\
9 & $\mathbf{3 1}$ & Chiralcel OJ-H & $95: 5$ & 24.4 & 26.2 \\
10 & $\mathbf{4}$ & Chiralpak IA & $98: 2$ & 6.1 & 6.4 \\
\hline
\end{tabular}

a Flow: $0.8 \mathrm{~mL} / \mathrm{min}$. Temperature: $30^{\circ} \mathrm{C}$. 


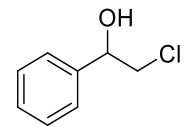

$3 a$

HPLC separation for both enantiomers of racemic alcohol 3a

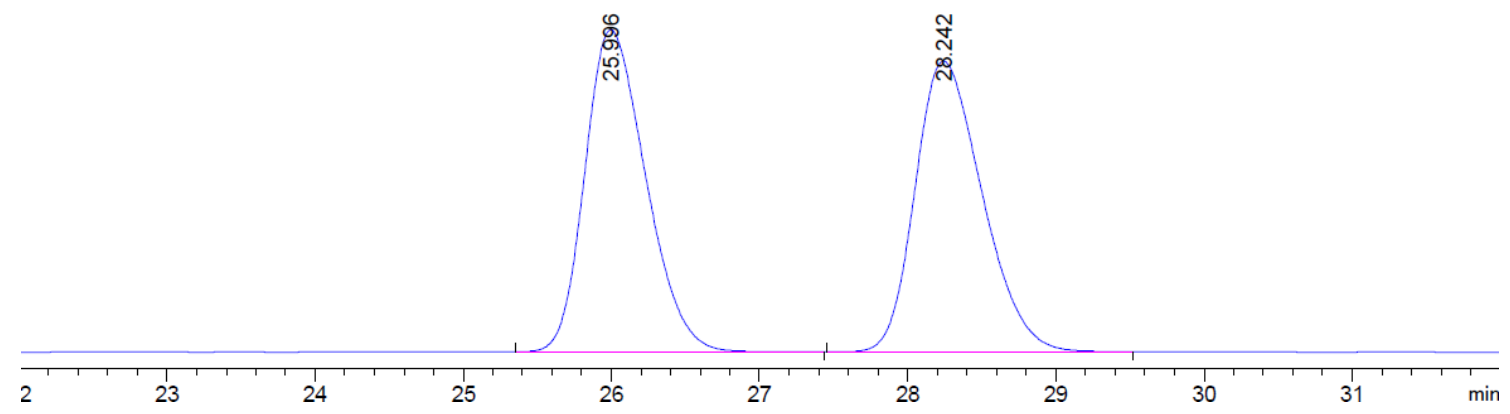

Alcohol $(S)-3 \mathbf{a}$ in $>99 \%$ ee (after bioreduction with $\mathrm{LbADH}$ )

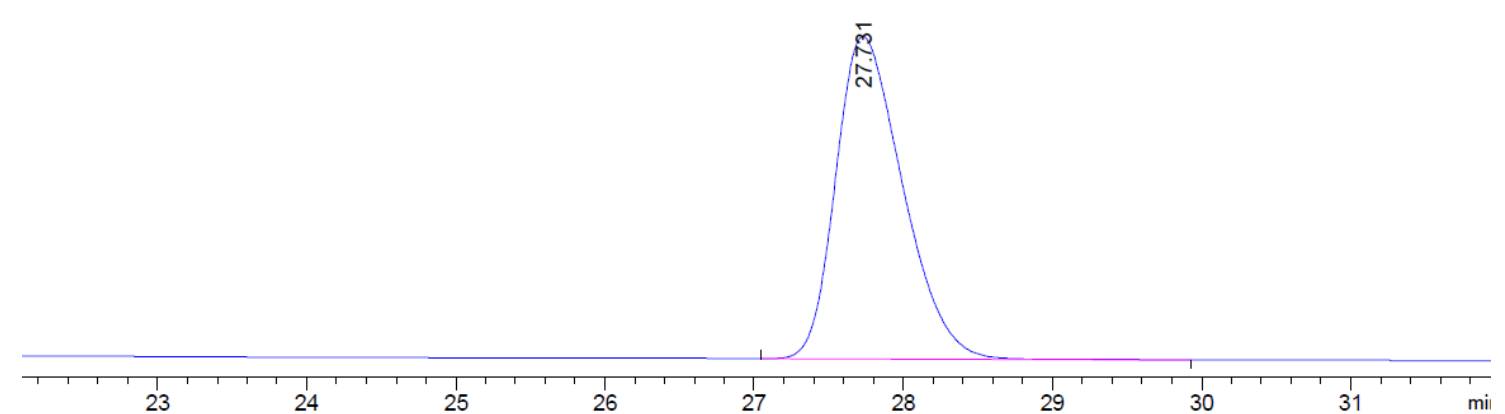

Alcohol $(R)-3 \mathbf{a}$ in $>99 \%$ ee (after bioreduction with ADH-A)

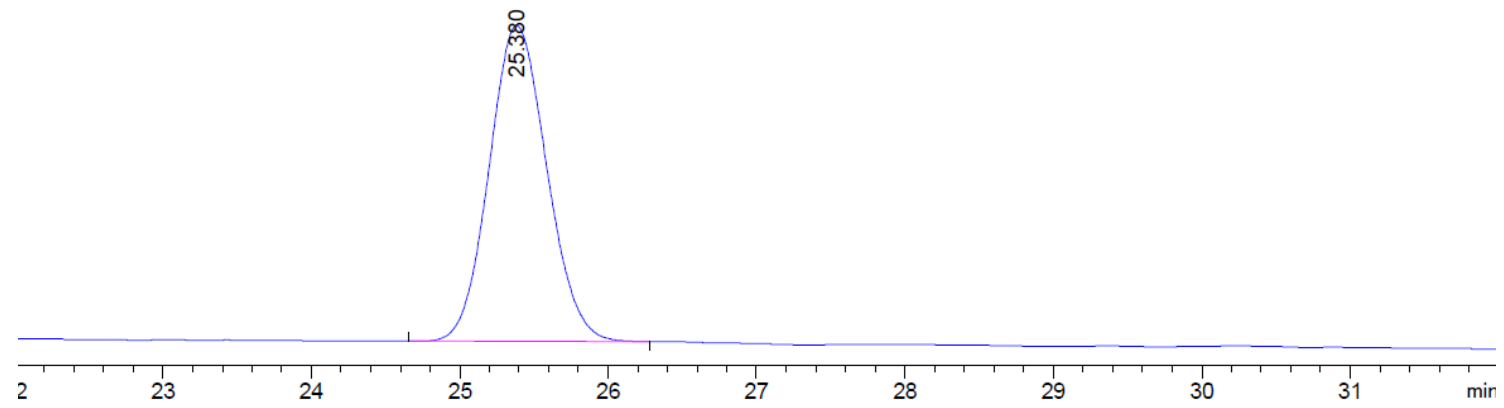

Figure S21. HPLC chromatograms of racemic halohydrin and optically active 3a obtained using selective ADHs. 


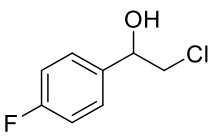

3b

HPLC separation for both enantiomers of racemic alcohol $\mathbf{3 b}$

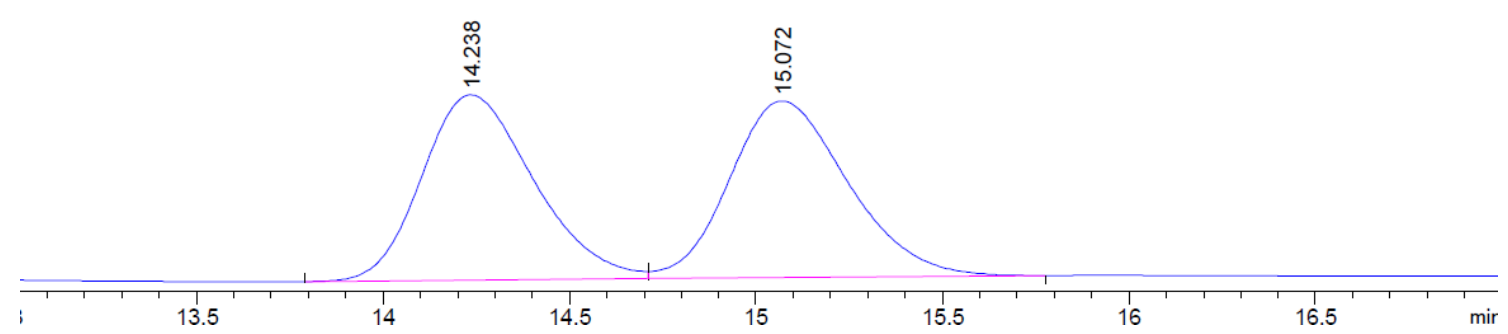

Alcohol $(S)-3 \mathbf{b}$ in $>99 \%$ ee (after bioreduction with $L b \mathrm{ADH})$

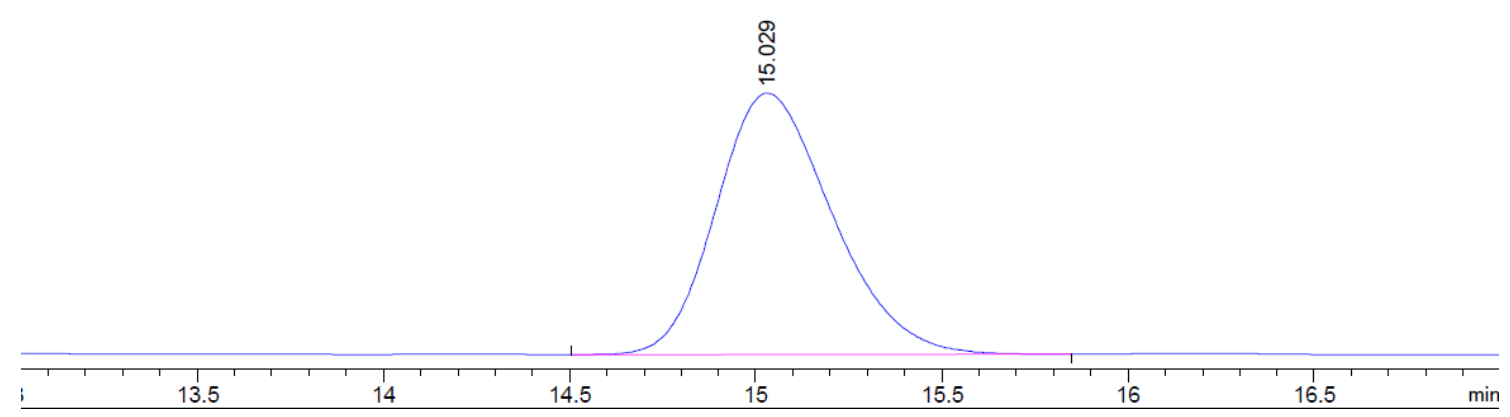

Alcohol $(R)-3 \mathbf{b}$ in $>99 \%$ ee (after bioreduction with ADH-A)



Figure S22. HPLC chromatograms of racemic halohydrin and optically active $\mathbf{3 b}$ obtained using selective ADHs. 


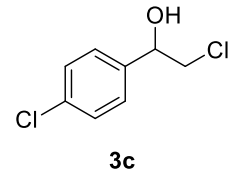

HPLC separation for both enantiomers of racemic alcohol 3c

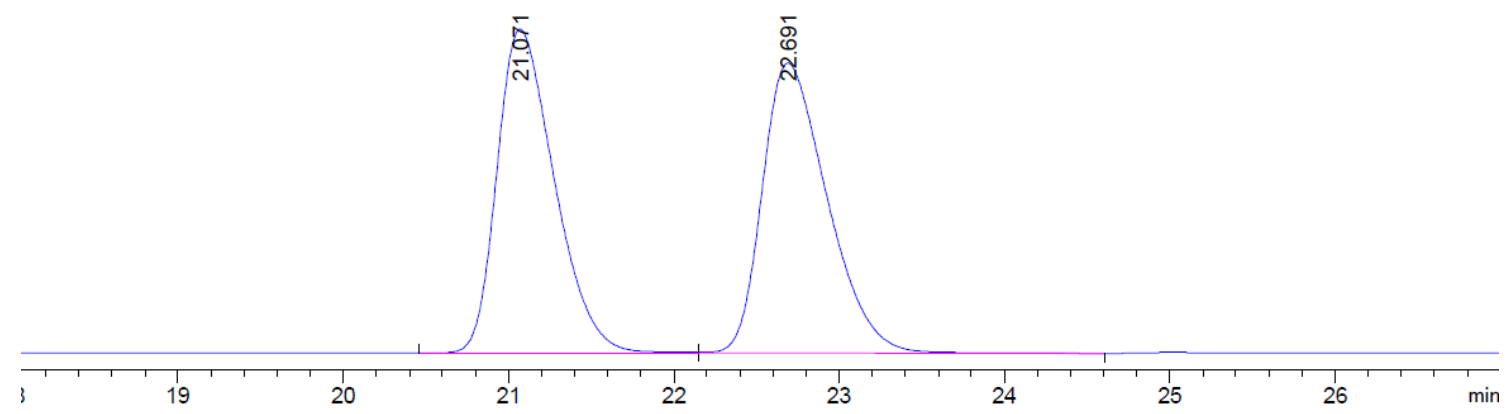

Alcohol $(S)-3 \mathbf{c}$ in $>99 \%$ ee (after bioreduction with $\mathrm{LbADH}$ )

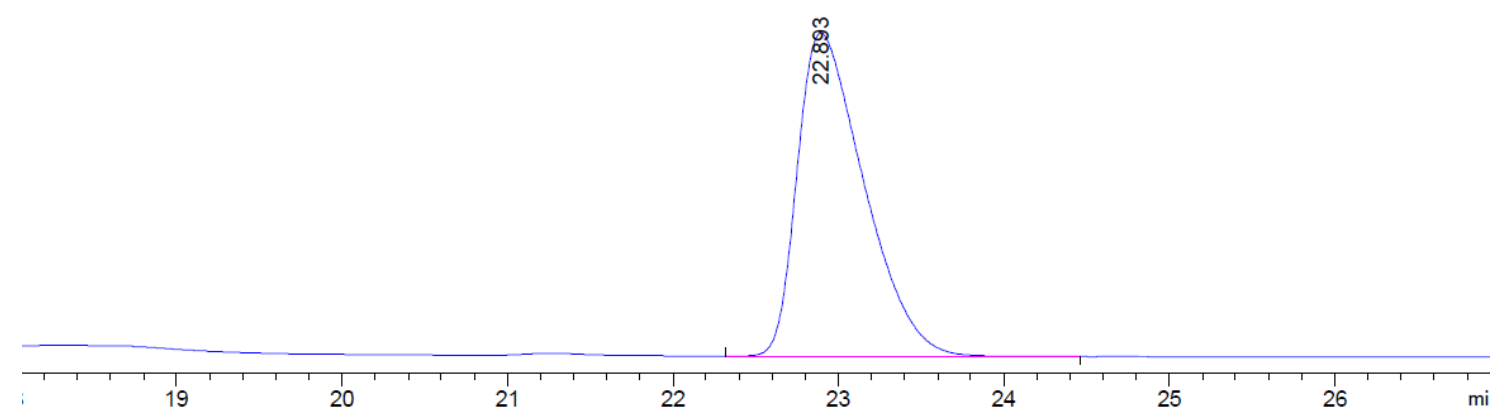

Alcohol $(R)-3 \mathbf{c}$ in $>99 \%$ ee (after bioreduction with ADH-A)

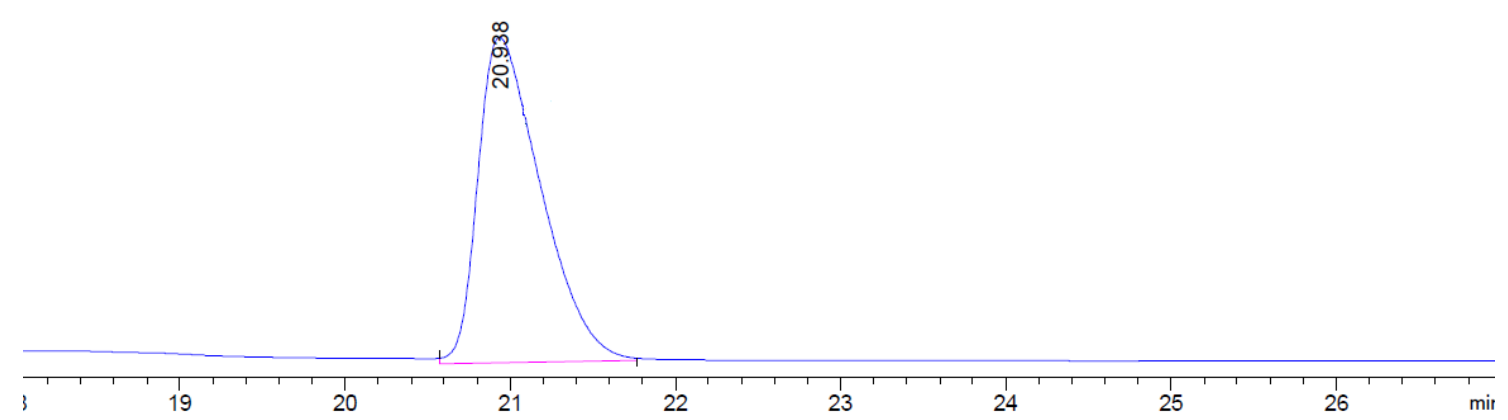

Figure S23. HPLC chromatograms of racemic halohydrin and optically active $\mathbf{3 c}$ obtained using selective ADHs. 


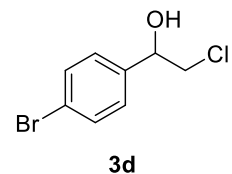

HPLC separation for both enantiomers of racemic alcohol 3d

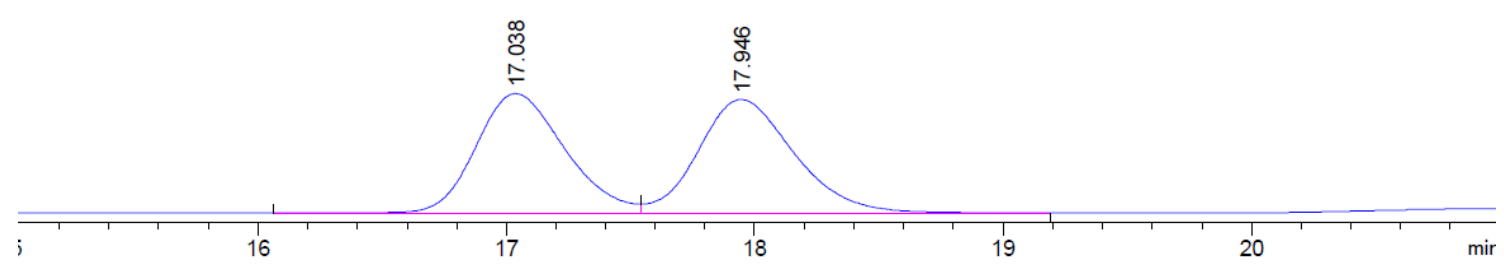

Alcohol $(S)-3 \mathbf{d}$ in $>99 \%$ ee (after bioreduction with $\mathrm{LbADH}$ )

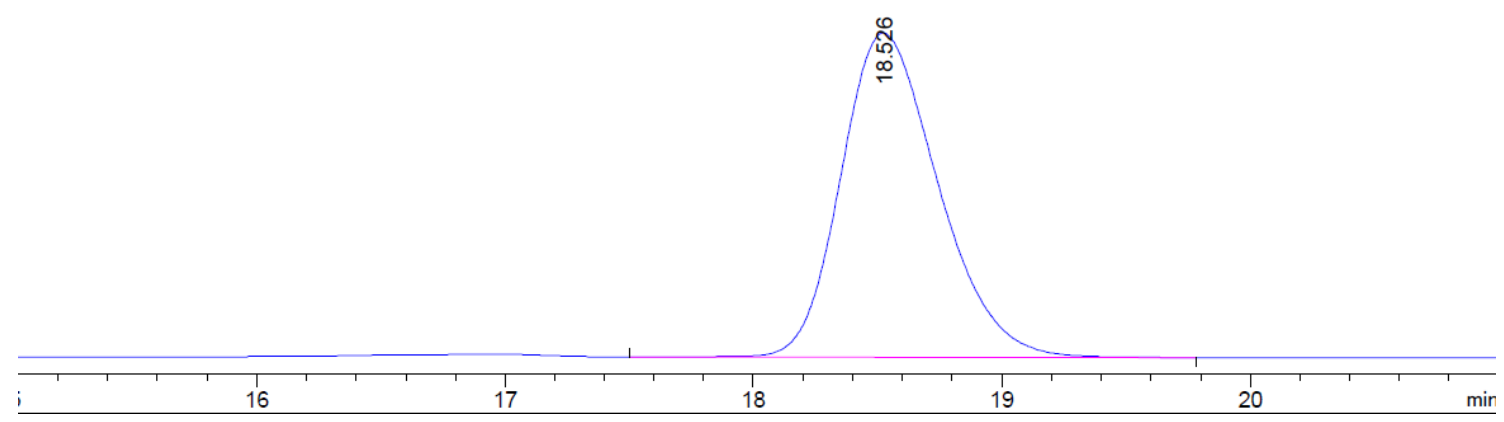

Alcohol ( $R$ )-3d in 99\% ee (after bioreduction with ADH-A)

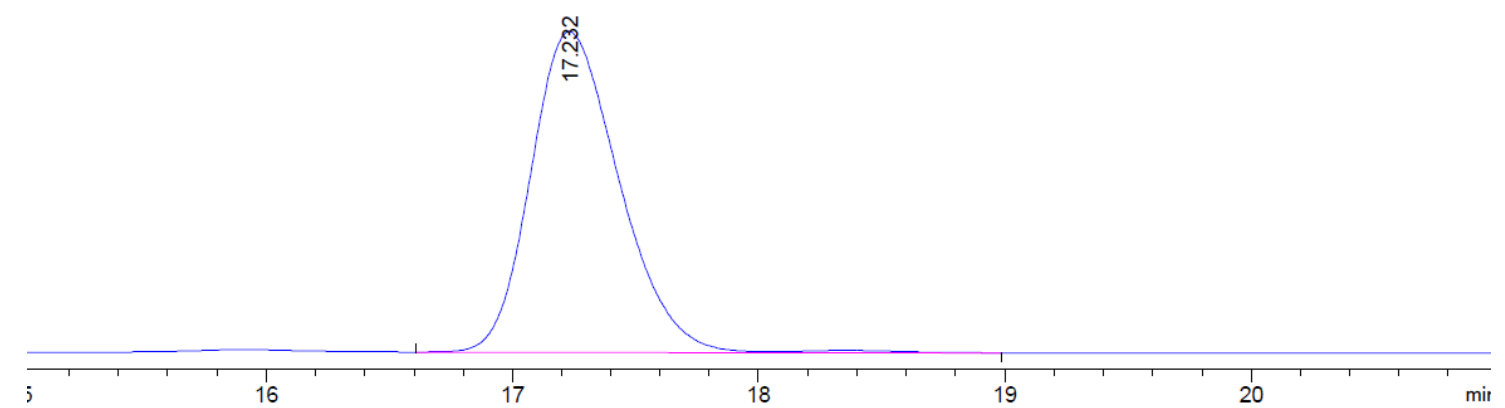

Figure S24. HPLC chromatograms of racemic halohydrin and optically active 3d obtained using selective ADHs. 


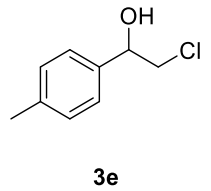

HPLC separation for both enantiomers of racemic alcohol 3e

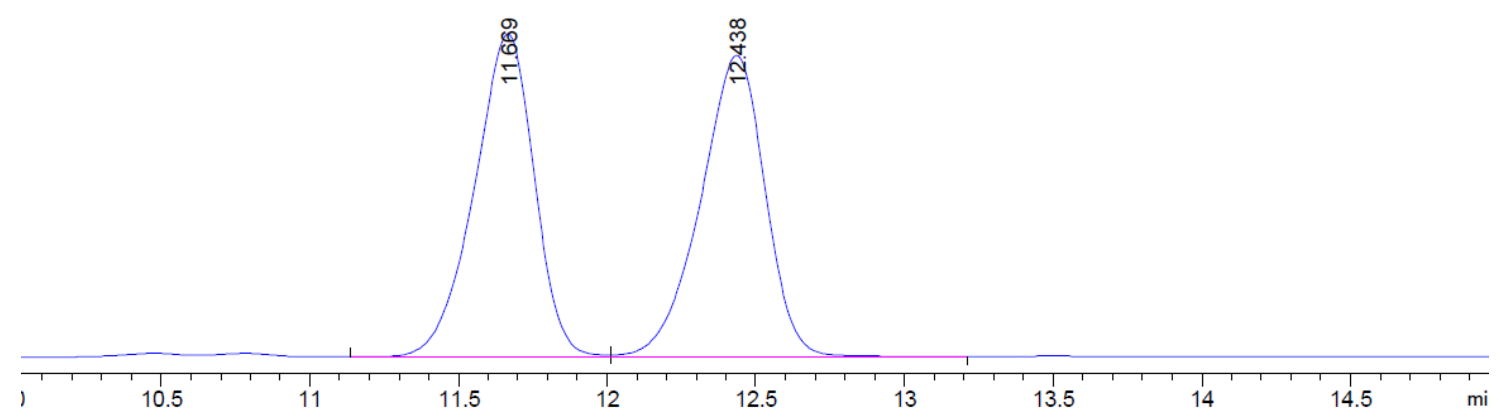

Alcohol $(S)-3 \mathbf{e}$ in $>99 \%$ ee (after bioreduction with $\mathrm{LbADH}$ )



Alcohol $(R)-3 e$ in $99 \%$ ee (after bioreduction with ADH-A)

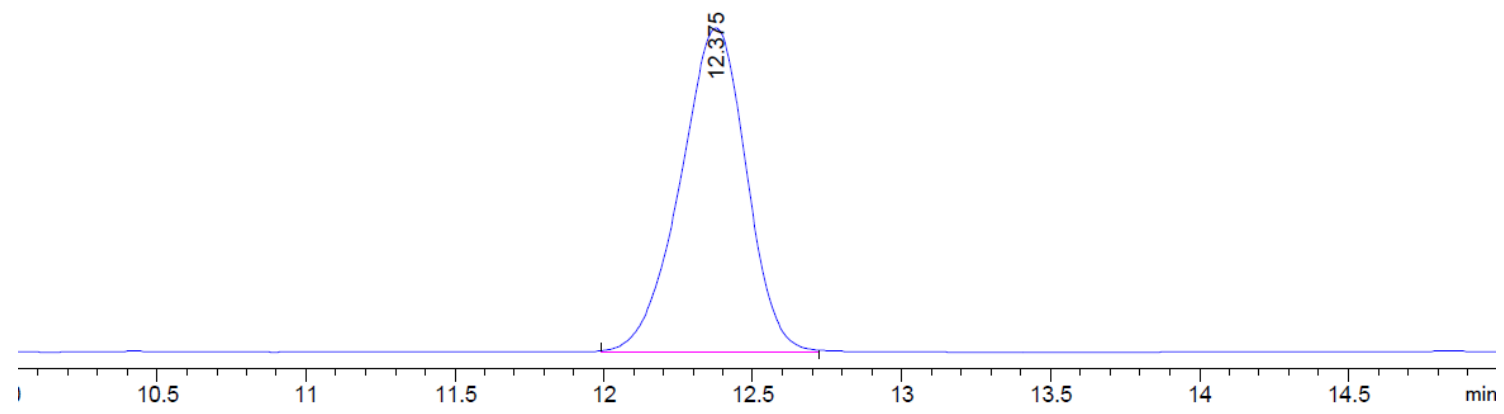

Figure S25. HPLC chromatograms of racemic halohydrin and optically active 3e obtained using selective ADHs. 


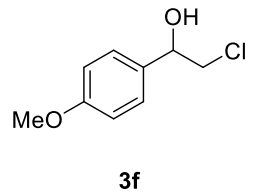

HPLC separation for both enantiomers of racemic alcohol $\mathbf{3 f}$

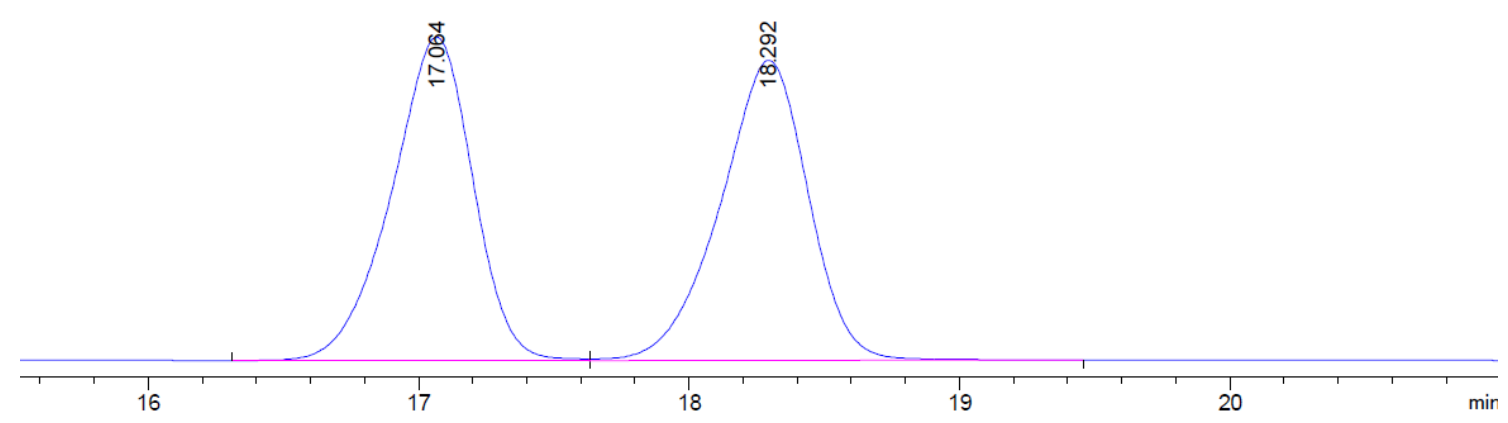

Alcohol $(S)-3 f$ in $>99 \%$ ee (after bioreduction with $\mathrm{LbADH}$ )



Alcohol $(R)-3 f$ in $99 \%$ ee (after bioreduction with ADH-A)

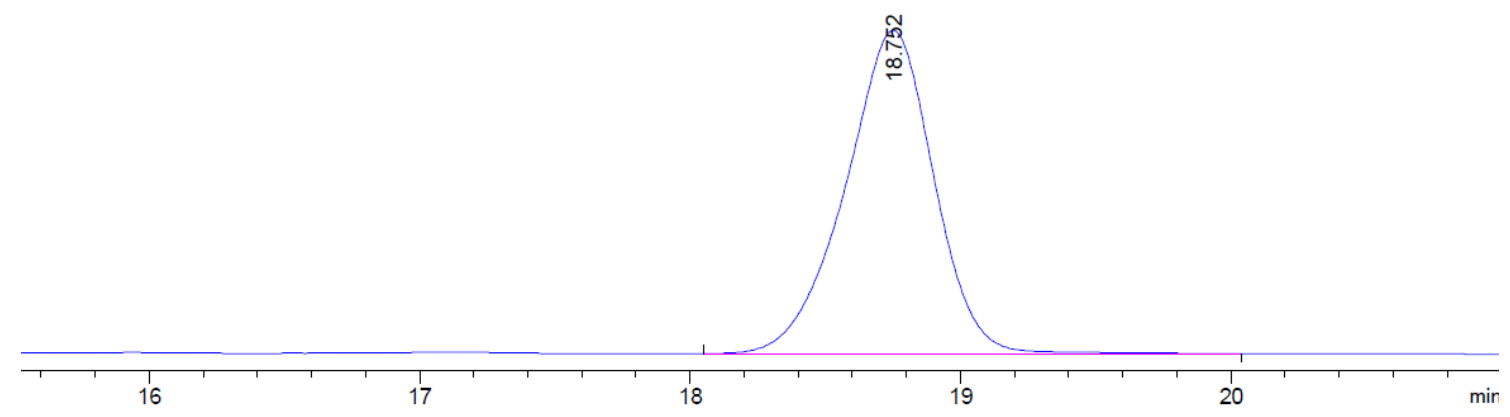

Figure S26. HPLC chromatograms of racemic halohydrin and optically active $\mathbf{3 f}$ obtained using selective ADHs. 


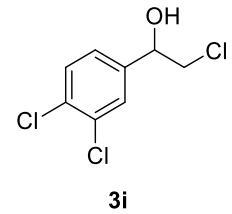

HPLC separation for both enantiomers of racemic alcohol $\mathbf{3 i}$

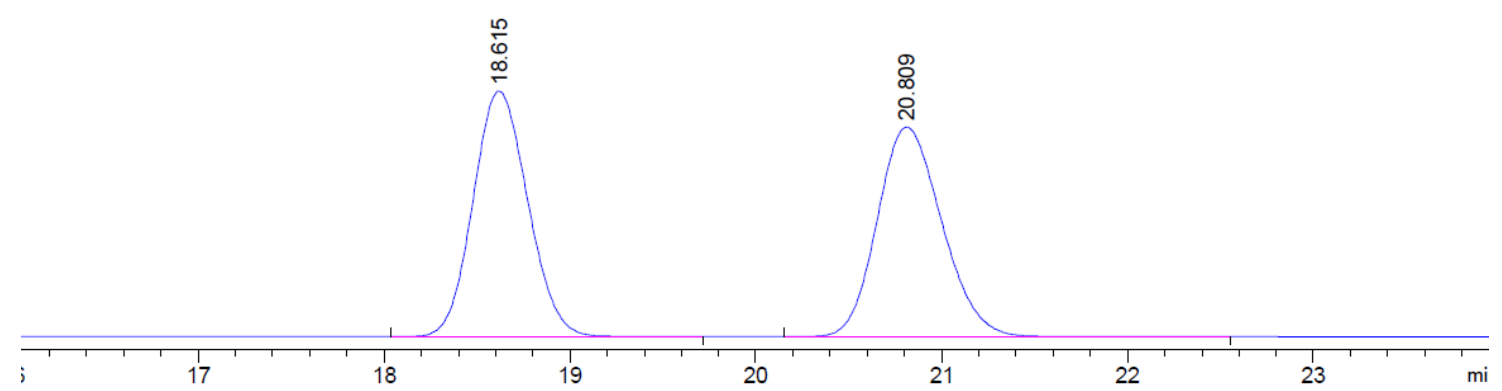

Alcohol (S)-3i in $>99 \%$ ee (after bioreduction with $L b \mathrm{ADH}$ )

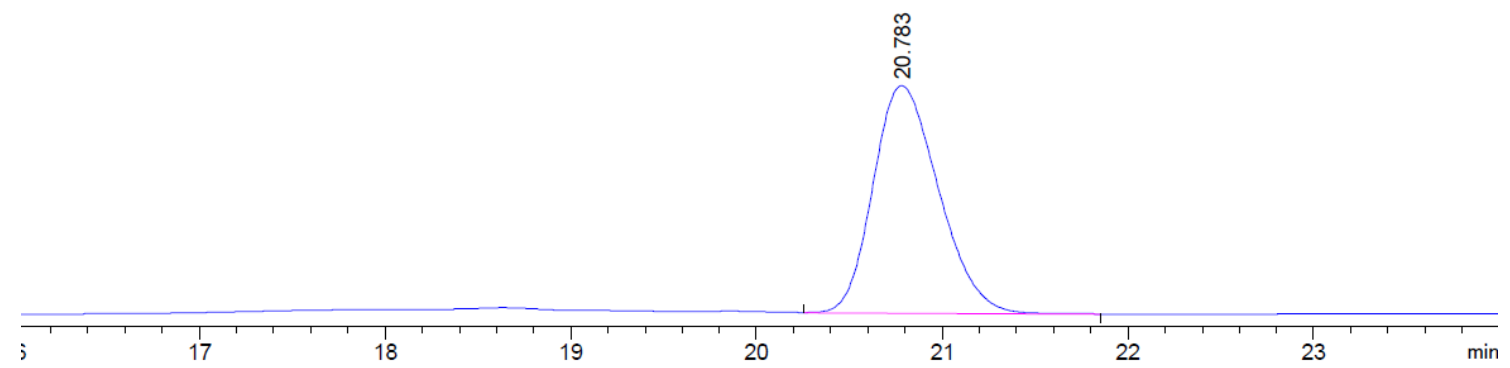

Alcohol $(R)-3 \mathbf{3}$ in $99 \%$ ee (after bioreduction with ADH-A)

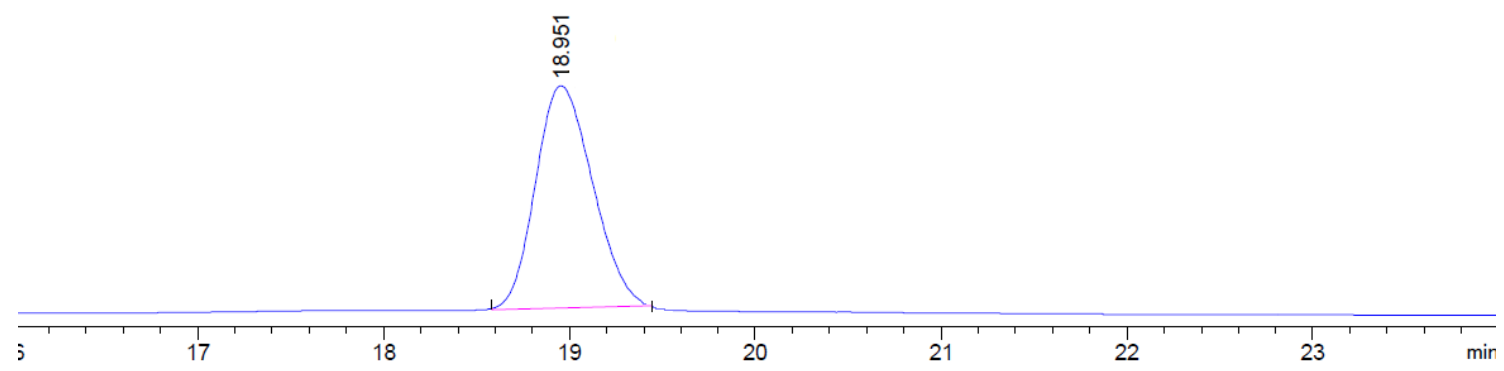

Figure S27. HPLC chromatograms of racemic halohydrin and optically active 3i obtained using selective ADHs. 


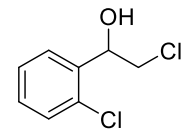

$3 \mathbf{j}$

HPLC separation for both enantiomers of racemic alcohol $\mathbf{3 j}$



Alcohol (S)-3j in 99\% ee (after bioreduction with KRED-P1-A04)

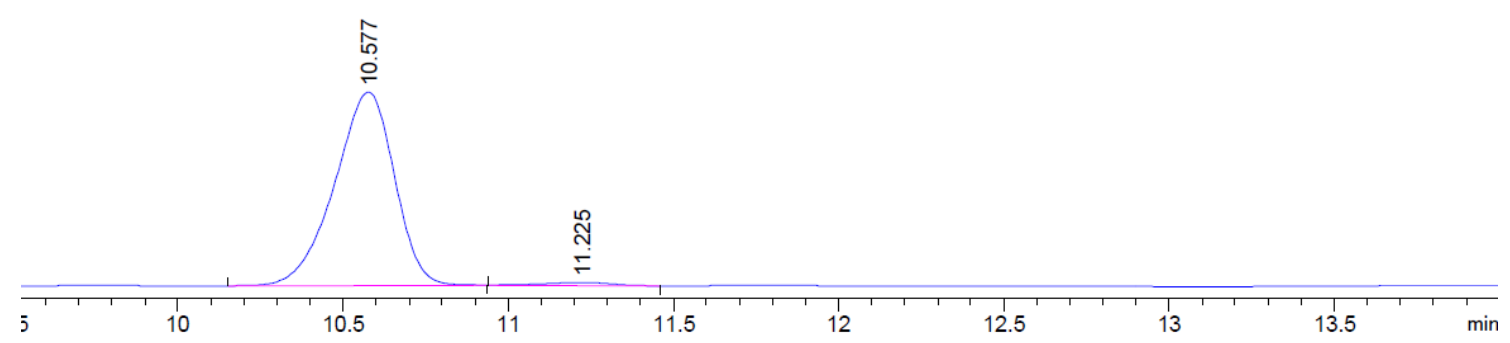

Alcohol $(R)-3 \mathbf{j}$ in 99\% ee (after bioreduction with KRED-P1-H08)

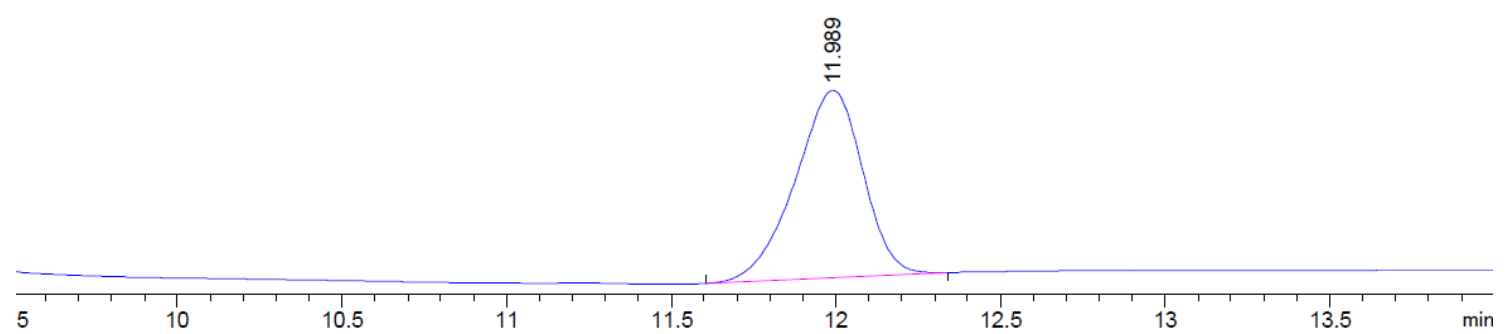

Figure S28. HPLC chromatograms of racemic halohydrin and optically active $\mathbf{3 j}$ obtained using selective ADHs. 


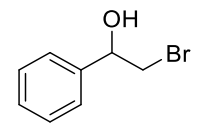

31

HPLC separation for both enantiomers of racemic alcohol 31

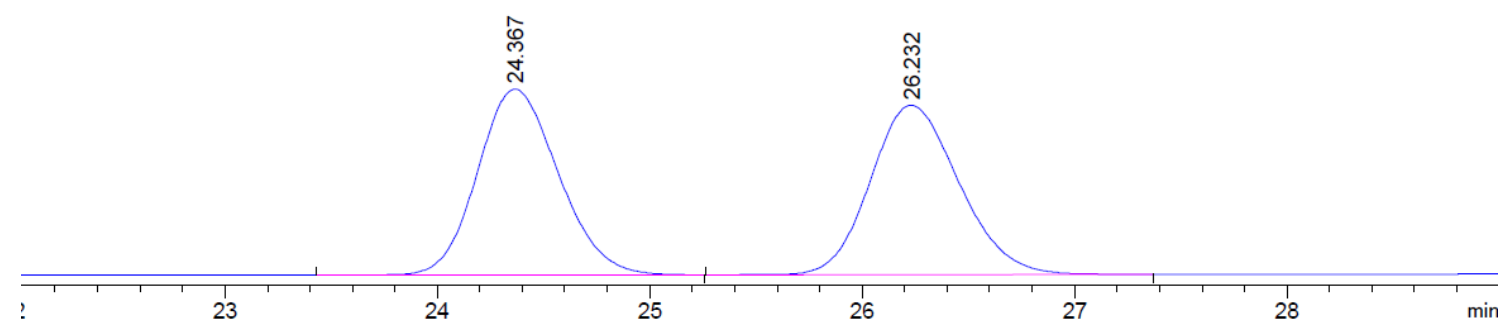

Alcohol $(S)-31$ in $>99 \%$ ee (after bioreduction with $L b$ ADH)

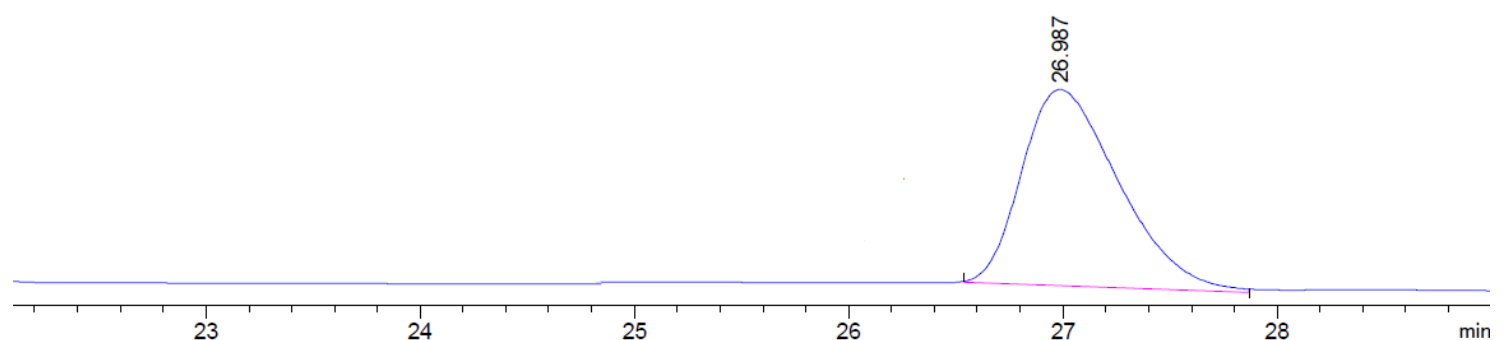

Figure S29. HPLC chromatograms of racemic halohydrin and optically active $\mathbf{3 l}$ obtained using $\mathrm{LbADH}$. 


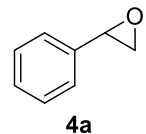

HPLC separation for both enantiomers of racemic styrene oxide $\mathbf{4 a}$

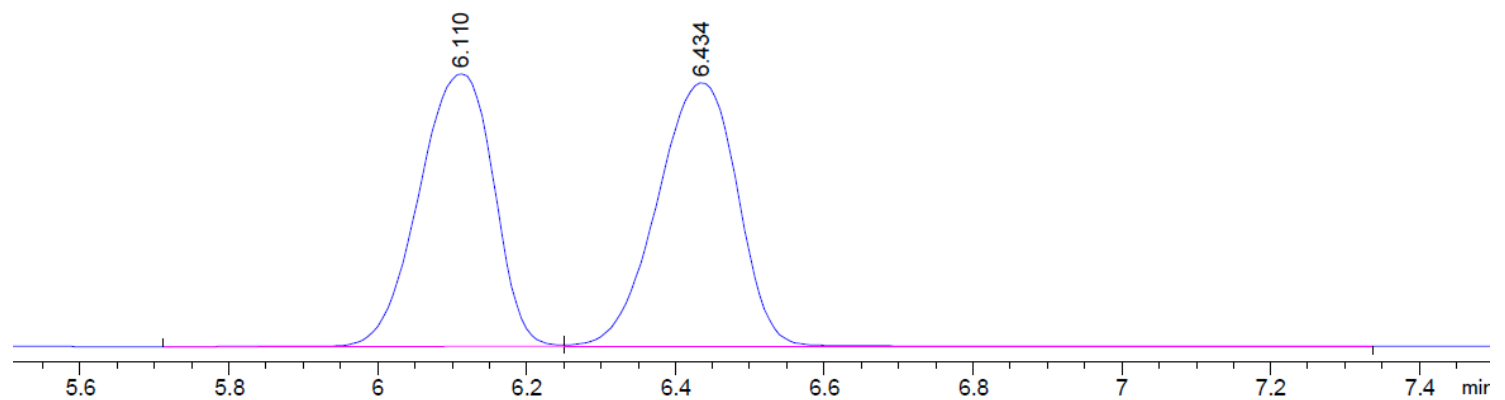

Styrene oxide $(S)-\mathbf{4 a}$ in $99 \%$ ee (after bioreduction with $L b \mathrm{ADH}$ )

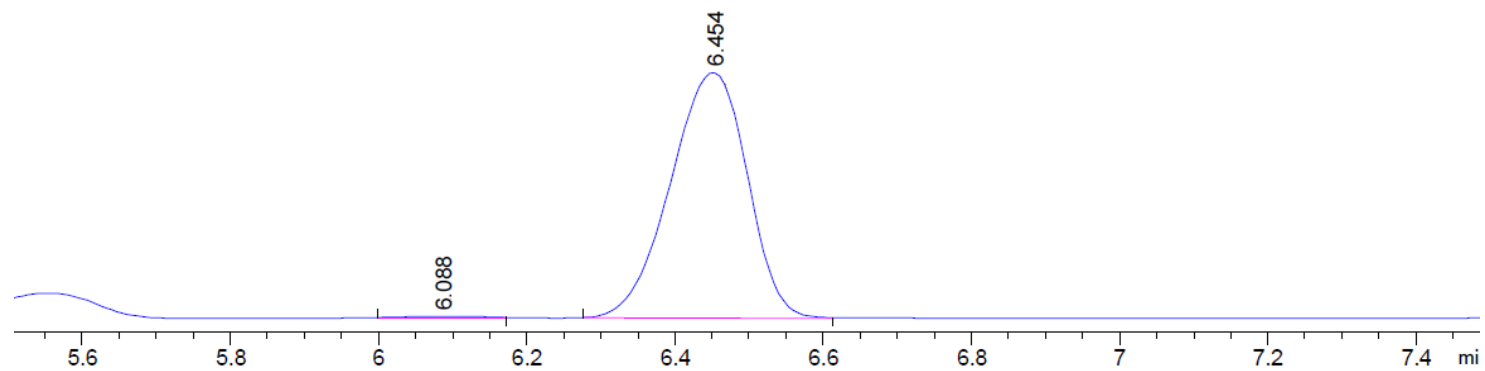

Styrene oxide $(R)-4 a$ in $96 \%$ ee (after bioreduction with ADH-A)



Figure S30. HPLC chromatograms of racemic epoxide and optically active 4 a obtained using selective $\mathrm{ADHs}$ in the reaction sequence. 
XV. Optical rotation values of halohydrins 3a-l obtained through the concurrent cascade approach

Table S17. Specific rotation of chiral halohydrins obtained through the gold(I)/ADH cascade.

\begin{tabular}{|c|c|c|c|c|}
\hline Entry & Enzyme & Compound & $e e(\%)^{\mathrm{a}}$ & Experimental $[\alpha]_{D}^{20}$ \\
\hline 1 & $L b \mathrm{ADH}$ & $\mathbf{3 a}$ & $>99^{\mathrm{b}}(S)$ & $+47.0\left(0.1 c, \mathrm{CHCl}_{3}\right)^{\mathrm{d}}$ \\
\hline 2 & ADH-A & $3 a$ & $>99^{\mathrm{b}}(R)$ & $-45.7\left(0.1 c, \mathrm{CHCl}_{3}\right)^{\mathrm{d}}$ \\
\hline 3 & $L b \mathrm{ADH}$ & $3 \mathbf{b}$ & $>99^{\mathrm{b}}(S)$ & $+48.9\left(0.1 c, \mathrm{CHCl}_{3}\right)^{\mathrm{d}}$ \\
\hline 4 & ADH-A & $\mathbf{3 b}$ & $>99^{\mathrm{b}}(R)$ & $-48.5\left(0.1 c, \mathrm{CHCl}_{3}\right)^{\mathrm{d}}$ \\
\hline 5 & LbADH & $3 c$ & $>99^{c}(S)$ & $+46.9\left(0.1 c, \mathrm{CHCl}_{3}\right)^{\mathrm{d}}$ \\
\hline 6 & $\mathrm{ADH}-\mathrm{A}$ & $3 c$ & $>99^{\mathrm{c}}(R)$ & $-47.3\left(0.1 c, \mathrm{CHCl}_{3}\right)^{\mathrm{d}}$ \\
\hline 7 & LbADH & 3d & $>99^{\mathrm{c}}(S)$ & $+43.7\left(0.1 c, \mathrm{CHCl}_{3}\right)^{\mathrm{d}}$ \\
\hline 8 & $\mathrm{ADH}-\mathrm{A}$ & 3d & $>99^{\mathrm{c}}(R)$ & $-42.3\left(0.1 c, \mathrm{CHCl}_{3}\right)^{\mathrm{d}}$ \\
\hline 9 & LbADH & $3 e$ & $>99^{\mathrm{c}}(S)$ & $+45.9\left(0.1 c, \mathrm{CHCl}_{3}\right)^{\mathrm{d}}$ \\
\hline 10 & ADH-A & $3 e$ & $>99^{\mathrm{c}}(R)$ & $-46.7\left(0.1 c, \mathrm{CHCl}_{3}\right)^{\mathrm{d}}$ \\
\hline 11 & $L b \mathrm{ADH}$ & $3 f$ & $>99^{\mathrm{c}}(S)$ & $+50.3\left(0.1 c, \mathrm{CHCl}_{3}\right)^{\mathrm{e}}$ \\
\hline 12 & $\mathrm{ADH}-\mathrm{A}$ & $3 f$ & $>99^{\mathrm{c}}(R)$ & $-49.6\left(0.1 c, \mathrm{CHCl}_{3}\right)^{\mathrm{e}}$ \\
\hline 13 & $L b \mathrm{ADH}$ & $3 g$ & $99^{\mathrm{b}}(S)$ & $+43.7\left(0.1 c, \mathrm{CHCl}_{3}\right)^{\mathrm{f}}$ \\
\hline 14 & ADH-A & $3 g$ & $>99^{\mathrm{b}}(R)$ & $-41.2\left(0.1 c, \mathrm{CHCl}_{3}\right)^{\mathrm{f}}$ \\
\hline 15 & $\mathrm{LbADH}$ & $3 \mathrm{~h}$ & $>99^{\mathrm{b}}(S)$ & $+42.0\left(0.1 c, \mathrm{CHCl}_{3}\right)^{\mathrm{g}}$ \\
\hline 16 & ADH-A & $3 \mathbf{h}$ & $>99^{\mathrm{b}}(R)$ & $-43.5\left(0.1 c, \mathrm{CHCl}_{3}\right)^{\mathrm{g}}$ \\
\hline 17 & $L b \mathrm{ADH}$ & $3 \mathbf{i}$ & $99^{\mathrm{c}}(S)$ & $+35.0\left(0.1 c, \mathrm{CHCl}_{3}\right)^{\mathrm{h}}$ \\
\hline 18 & ADH-A & $3 \mathbf{i}$ & $>99^{\mathrm{c}}(R)$ & $-33.8\left(0.1 c, \mathrm{CHCl}_{3}\right)^{\mathrm{h}}$ \\
\hline 19 & KRED-P1-A04 & $3 \mathbf{j}$ & $99^{\mathrm{c}}(S)$ & $+52.3\left(0.1 c, \mathrm{CHCl}_{3}\right)^{\mathrm{i}}$ \\
\hline 20 & KRED-P1-H08 & $\mathbf{3 j}$ & $>99^{\mathrm{c}}(R)$ & $-55.8\left(0.1 c, \mathrm{CHCl}_{3}\right)^{\mathrm{i}}$ \\
\hline 21 & $L b \mathrm{ADH}$ & $3 \mathbf{k}$ & $>99^{\mathrm{c}}(S)$ & $+2.4\left(0.1 c, \mathrm{CHCl}_{3}\right)^{\mathrm{j}}$ \\
\hline 22 & ADH-A & $3 \mathbf{k}$ & $99^{\mathrm{c}}(R)$ & $-3.5\left(0.1 c, \mathrm{CHCl}_{3}\right)^{\mathrm{j}}$ \\
\hline 23 & ADH-T & $3 \mathbf{k}$ & $>99^{\mathrm{c}}(R)$ & $-2.8\left(0.1 c, \mathrm{CHCl}_{3}\right)^{\mathrm{j}}$ \\
\hline 24 & $L b \mathrm{ADH}$ & 31 & $>99^{\mathrm{c}}(S)$ & $+51.7\left(0.1 c, \mathrm{CHCl}_{3}\right)^{\mathrm{d}}$ \\
\hline
\end{tabular}

${ }^{a}$ Absolute configuration of the allylic alcohol 3a-l in parentheses.

${ }^{\mathrm{b}}$ Enantiomeric excess values were measured by GC analysis.

${ }^{\mathrm{c}}$ Enantiomeric excess values were measured by HPLC analysis

${ }^{\mathrm{d}}$ Optical rotation values were compared with those already described in the literature. ${ }^{7}$

${ }^{\mathrm{e}}$ Optical rotation values were compared with those already described in the literature. ${ }^{8}$

${ }^{f}$ Optical rotation values were compared with those already described in the literature. ${ }^{9}$

${ }^{\mathrm{g}}$ Optical rotation values were compared with those already described in the literature. ${ }^{10}$

${ }^{\mathrm{h}}$ Optical rotation values were compared with those already described in the literature. ${ }^{11}$

${ }^{i}$ Optical rotation values were compared with those already described in the literature. ${ }^{12}$

${ }^{\mathrm{j}}$ Optical rotation values were compared with those already described in the literature. ${ }^{13}$ 


\section{Reference section}

1. Nicolai, S.; Sedigh-Zadeh, R.; Waser, J. Pd(0)-Catalyzed Alkene Oxy- and Aminoalkynylation with Aliphatic Bromoacetylenes. J. Org. Chem. 2013, 78, 3783-3801.

2. McIntosh, M. L.; Johnston, R. C.; Pattawong, O.; Ashburn, B. O.; Naffziger, M. R.; Cheong, P. H.-Y.; Carter, R. G. Synthesis and Computational Analysis of Densely Functionalized Triazoles Using $o$-Nitrophenylalkynes. J. Org. Chem. 2012, 77, 1101-1112.

3. Wu, X.; Yang, Z.; Zhang, P.; Wang, L.; Guo, G.; Dong, Y.; Li, X. Polymerization of 1-Chloro2-Phenylacetylenes by Cationic Monomeric Tridentate $(S, S)$-Bis(oxazolinylphenyl)amidoLigated Palladium Catalysts: Is It Coordination-Insertion Mechanism?. Polym. Chem. 2018, 9, 4856-4865.

4. Chowdhury, R. M.; Wilden, J. D. An Improved Transition-Metal-Free Synthesis of Aryl Alkynyl Sulfides via Substitution of a Halide at an sp-Centre. Org. Biomol. Chem. 2015, 13, 58595861.

5. Shi, D.; Liu, Z.; Zhang, Z.; Shi, W.; Chen, H. Silver-Catalyzed Synthesis of 1-Chloroalkynes directly from Terminal Alkynes. ChemCatChem 2015, 7, 1424-1426.

6. Kreuzahler, M.; Haberhauer, G. Gold(I)-Catalyzed Chloroalkynylation of 1,1-Disubstituted Alkenes via 1,3-Chlorine Shift: A Combined Expertimental and Theoretical Study. J. Org. Chem. 2019, 84, 8210-8224.

7. Lin, H.; Chen, Y.-Z.; Xu, X.-Y.; Xia, S.-W.; Wang, L.-X. Preparation of Key Intermediates of Adrenergic Receptor Agonists: Highly Enantioselective Production of $(R)$ - $\alpha$-Halohydrins with Saccharomyces cerevisiae CGMCC 2.396. J. Mol. Catal. B: Enzym. 2009, 57, 1-5.

8. Huang, X.; Ying, J. Y. Asymmetric Transfer Hydrogenation over Ru-TsDPEN Catalysts Supported on Siliceous Mesocellular Foam. Chem. Commun. 2007, 1825-1827.

9. Zhu, D.; Mukherjee, C.; Hua, L. 'Green' Synthesis of Important Pharmaceutical Building Blocks: Enzymatic Access to Enantiomerically Pure $\alpha$-Chloroalcohols. Tetrahedron: Asymmetry 2005, 16, 3275-3278.

10. Hamada, T.; Torii, T.; Izawa, K.; Ikariya. T. A Practical Synthesis of Optically Active Aromatic Epoxides via Asymmetric Transfer Hydrogenation of $\alpha$-Chlorinated Ketones with Chiral Rhodium-Diamine Catalyst. Tetrahedron 2004, 60, 7411-7417.

11. Barros-Filho, B. A.; de Oliveira, M. C. F.; Lemos, T. L. G.; de Mattos, M. C.; de Gonzalo, G.; Gotor-Fernández, V.; Gotor, V. Lentinus strigellus: A New Versatile Stereoselective Biocatalyst for the Bioreduction of Prochiral Ketones. Tetrahedron: Asymmetry 2009, 20, 10571061.

12. Yin, C.; Wu, W.; Hu, Y.; Tan, X.; You, C.; Liu, Y.; Chen, Z.; Dong, X.-Q.; Zhang, X. IridiumCatalyzed Asymmetric Hydrogenation of Halogenated Ketones for the Efficient Construction of Chiral Halohydrins. Adv. Synth. Catal. 2018, 360, 2119-2124.

13. Poessl, T. M.; Kosjek, B.; Ellmer, U.; Gruber, C. C.; Edegger, K.; Faber, K.; Hildebrandt, P.; Bornscheuer, U. T.; Kroutil, W. Non-Racemic Halohydrins via Biocatalytic Hydrogen-Transfer Reduction of Halo-Ketones and One-Pot Cascade Reaction to Enantiopure Epoxides. Adv. Synth. Catal. 2005, 347, 1827-1834. 
XVII. NMR spectra
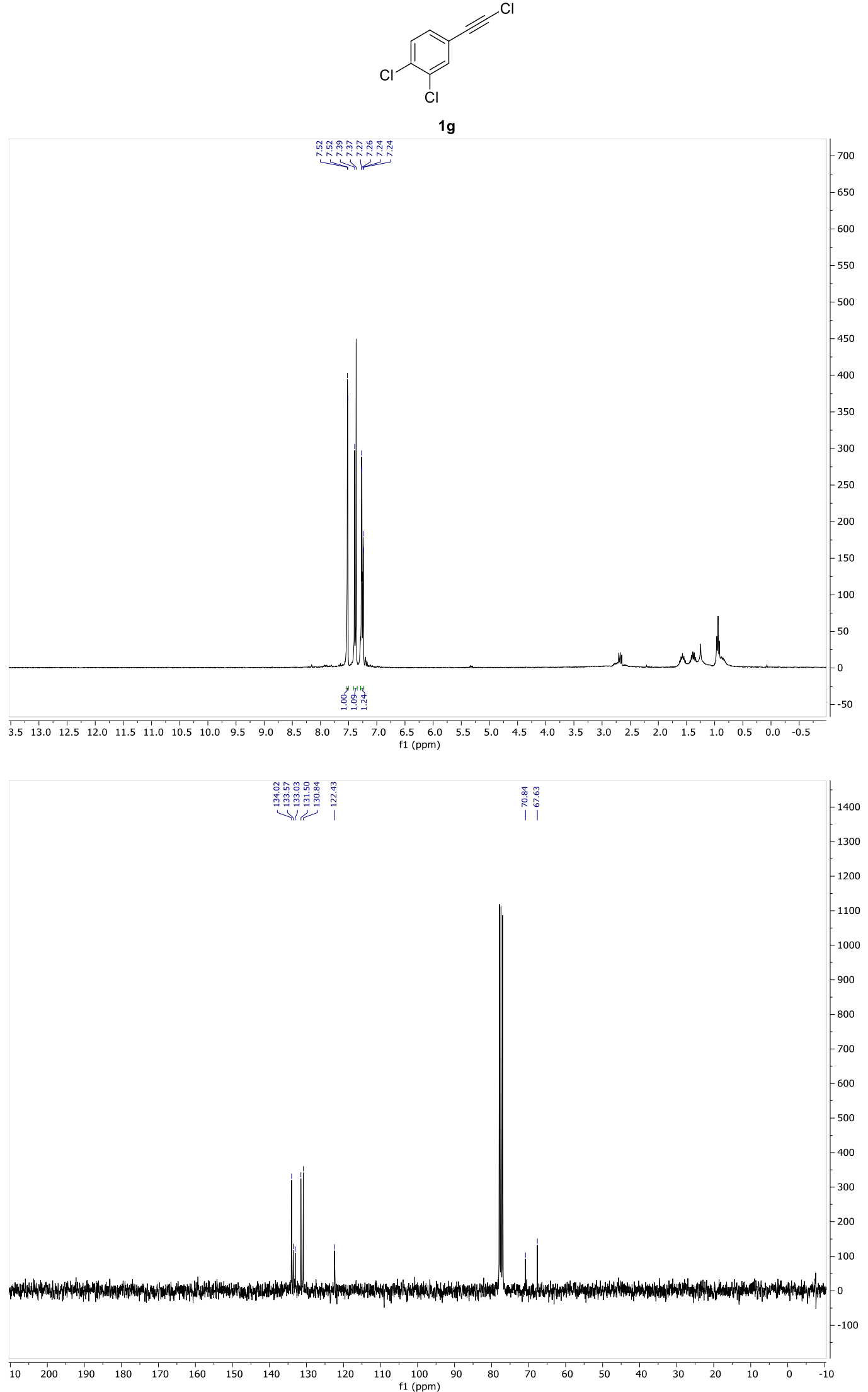

Figure S31. ${ }^{1} \mathrm{H}$ and ${ }^{13} \mathrm{C}$ NMR spectra $\left(\mathrm{CDCl}_{3}\right)$ of compound $\mathbf{1 g}$. 


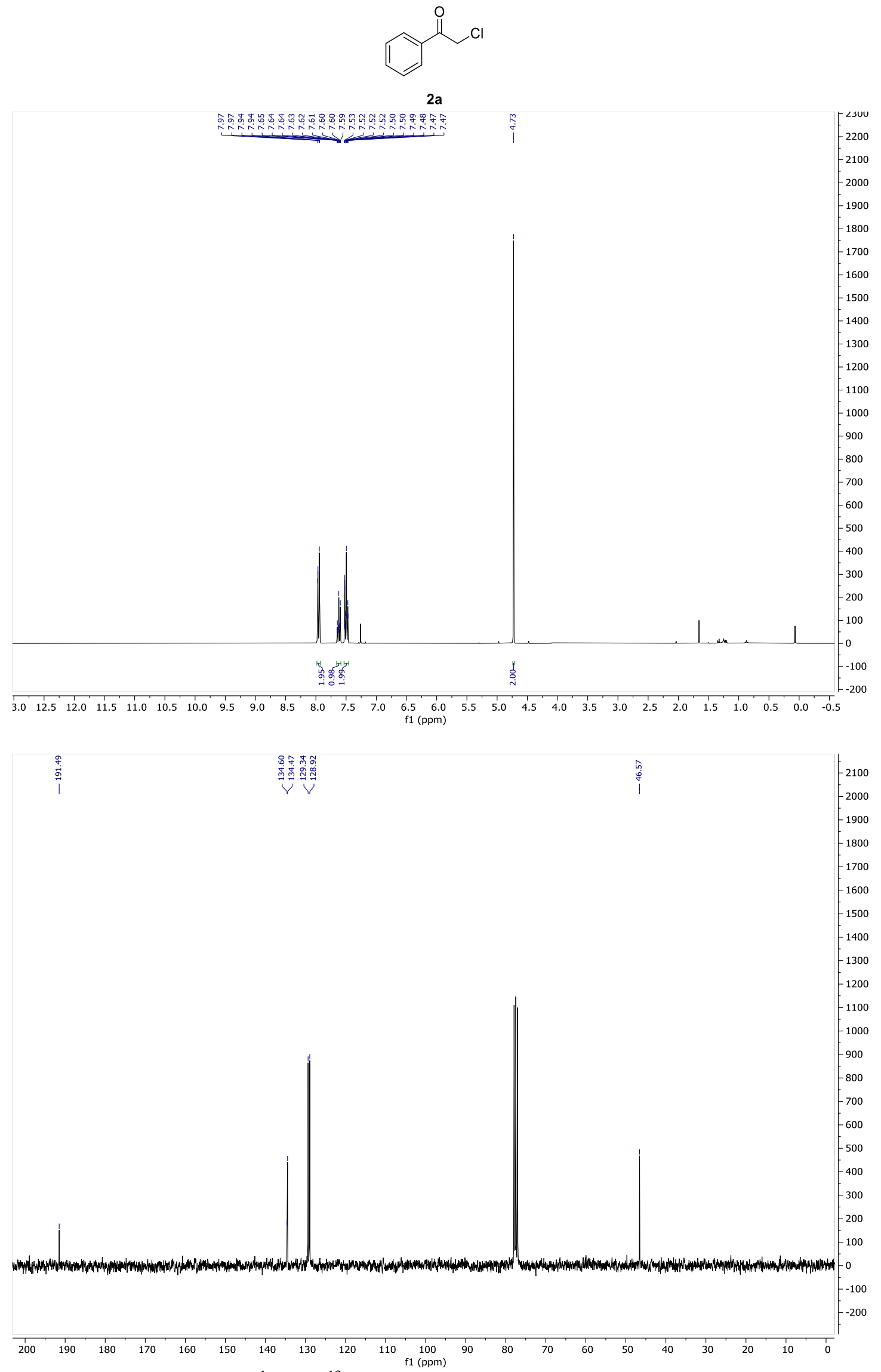

Figure S32. ${ }^{1} \mathrm{H}$ and ${ }^{13} \mathrm{C}$ NMR spectra $\left(\mathrm{CDCl}_{3}\right)$ of compound $2 \mathrm{a}$. 


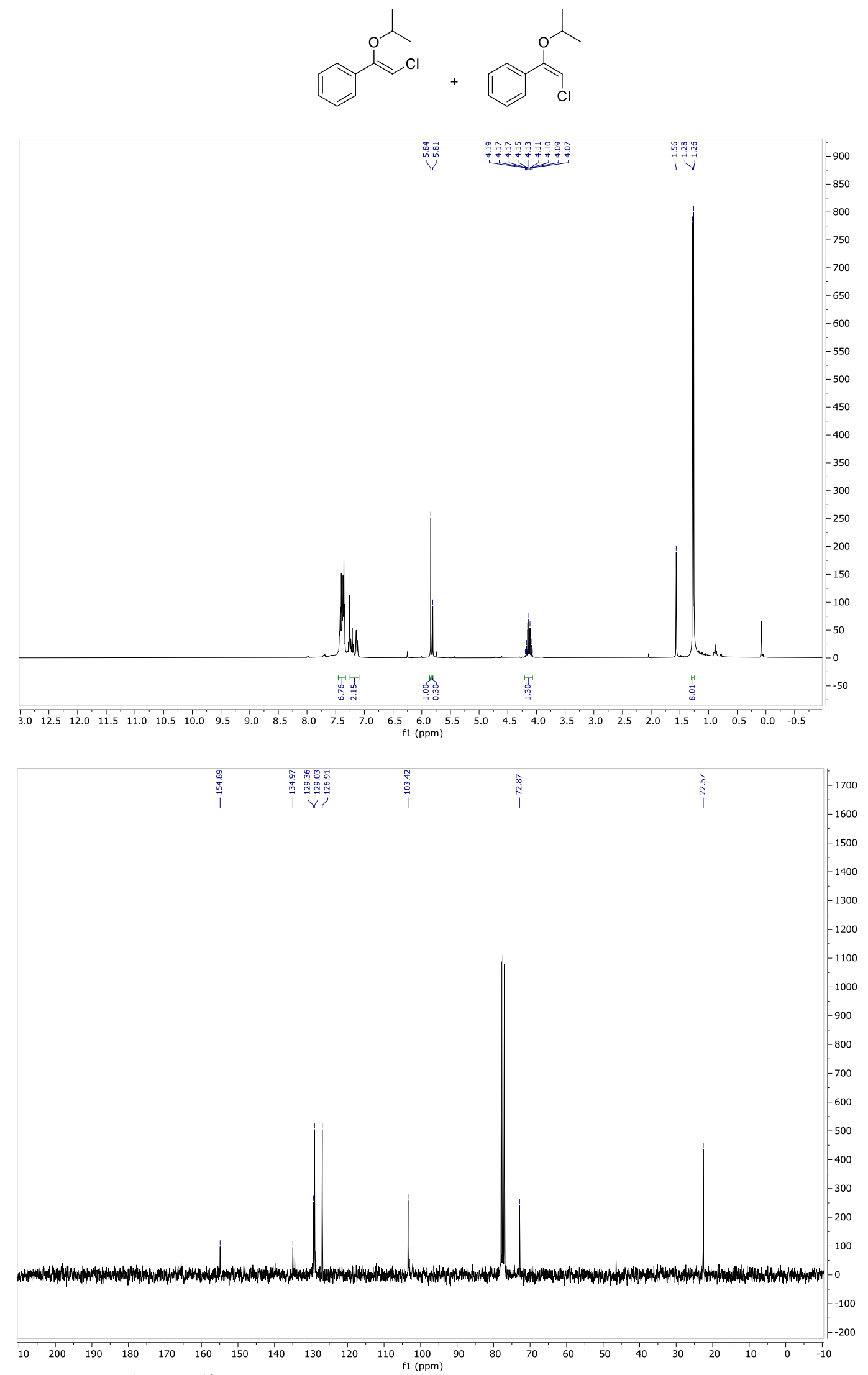

Figure S33. ${ }^{1} \mathrm{H}$ and ${ }^{13} \mathrm{C}$ NMR spectra $\left(\mathrm{CDCl}_{3}\right)$ of by-products detected in the hydration of $1 \mathrm{a}$. 


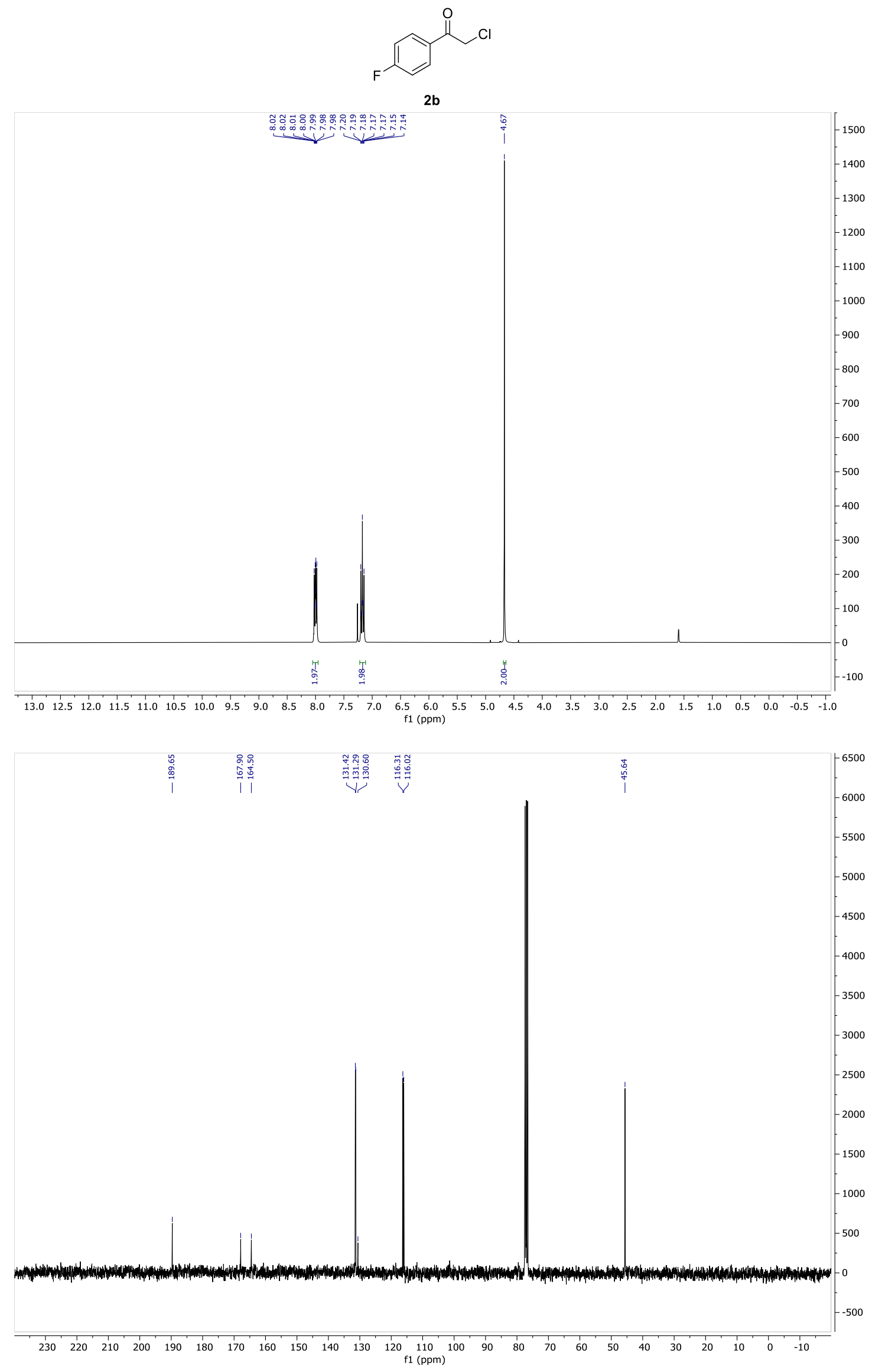




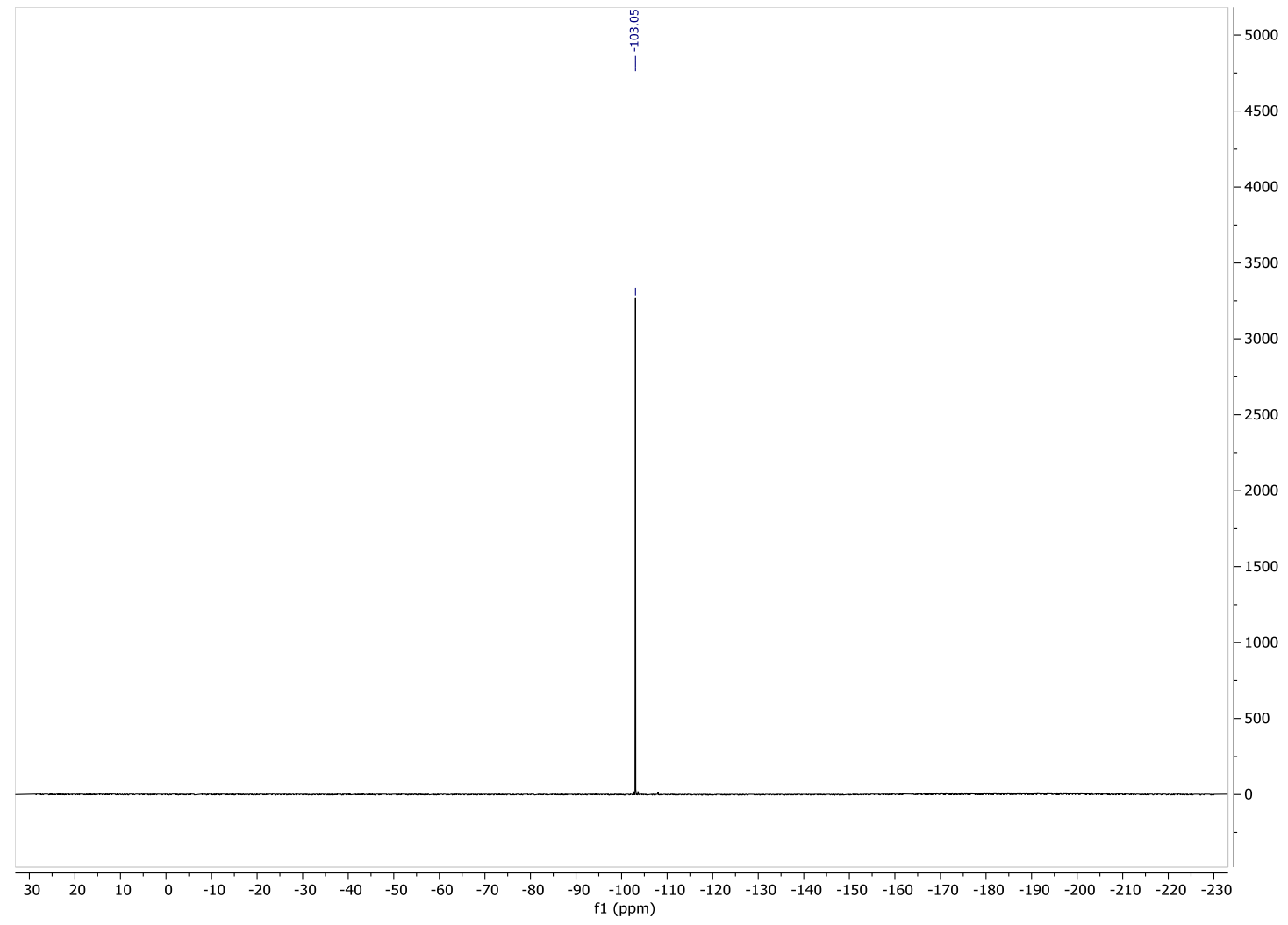

Figure S34. ${ }^{1} \mathrm{H},{ }^{13} \mathrm{C}$ and ${ }^{19} \mathrm{~F}$ NMR spectra $\left(\mathrm{CDCl}_{3}\right)$ of compound $\mathbf{2 b}$. 


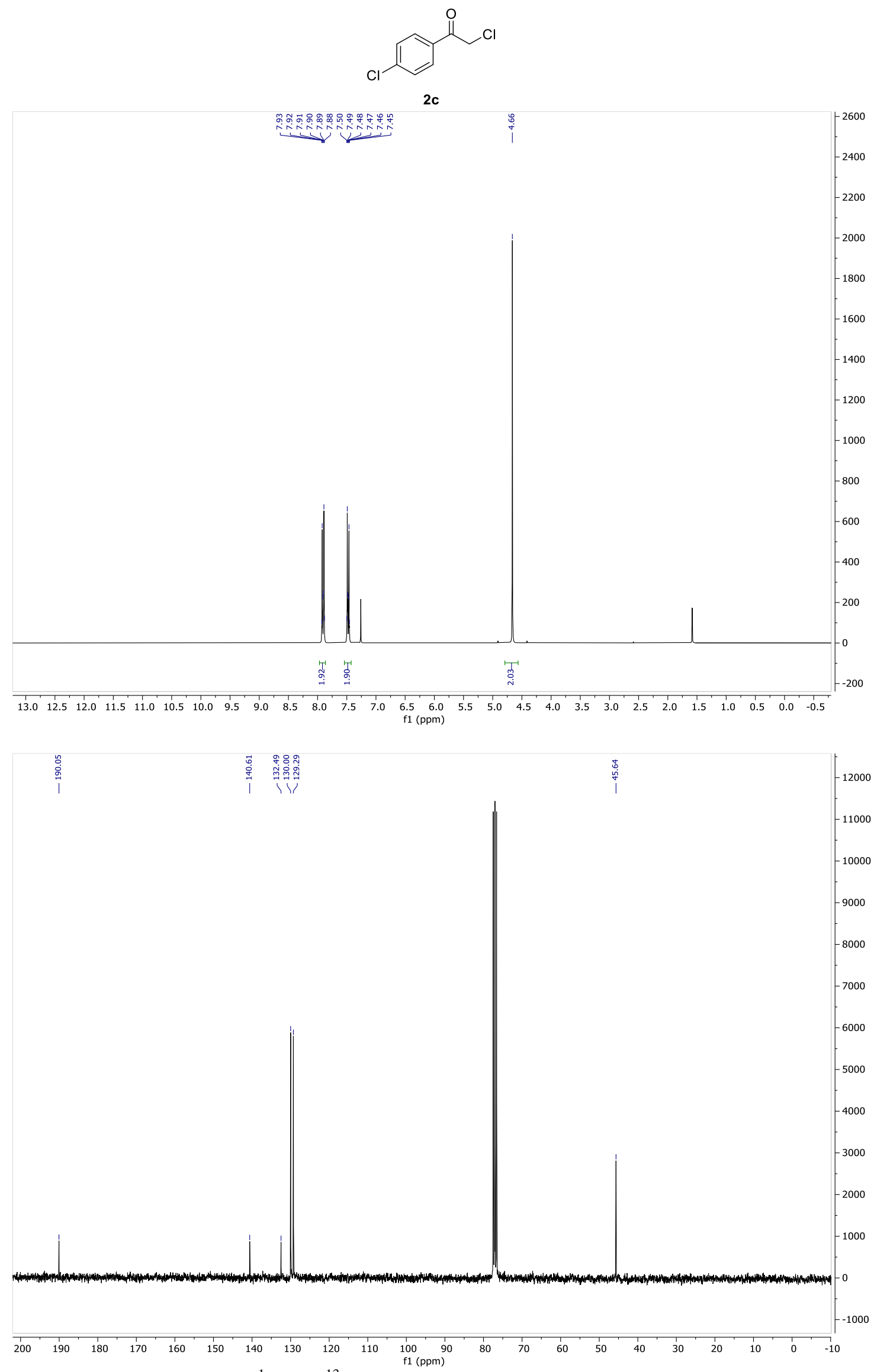

Figure S35. ${ }^{1} \mathrm{H}$ and ${ }^{13} \mathrm{C}$ NMR spectra $\left(\mathrm{CDCl}_{3}\right)$ of compound 2c. 


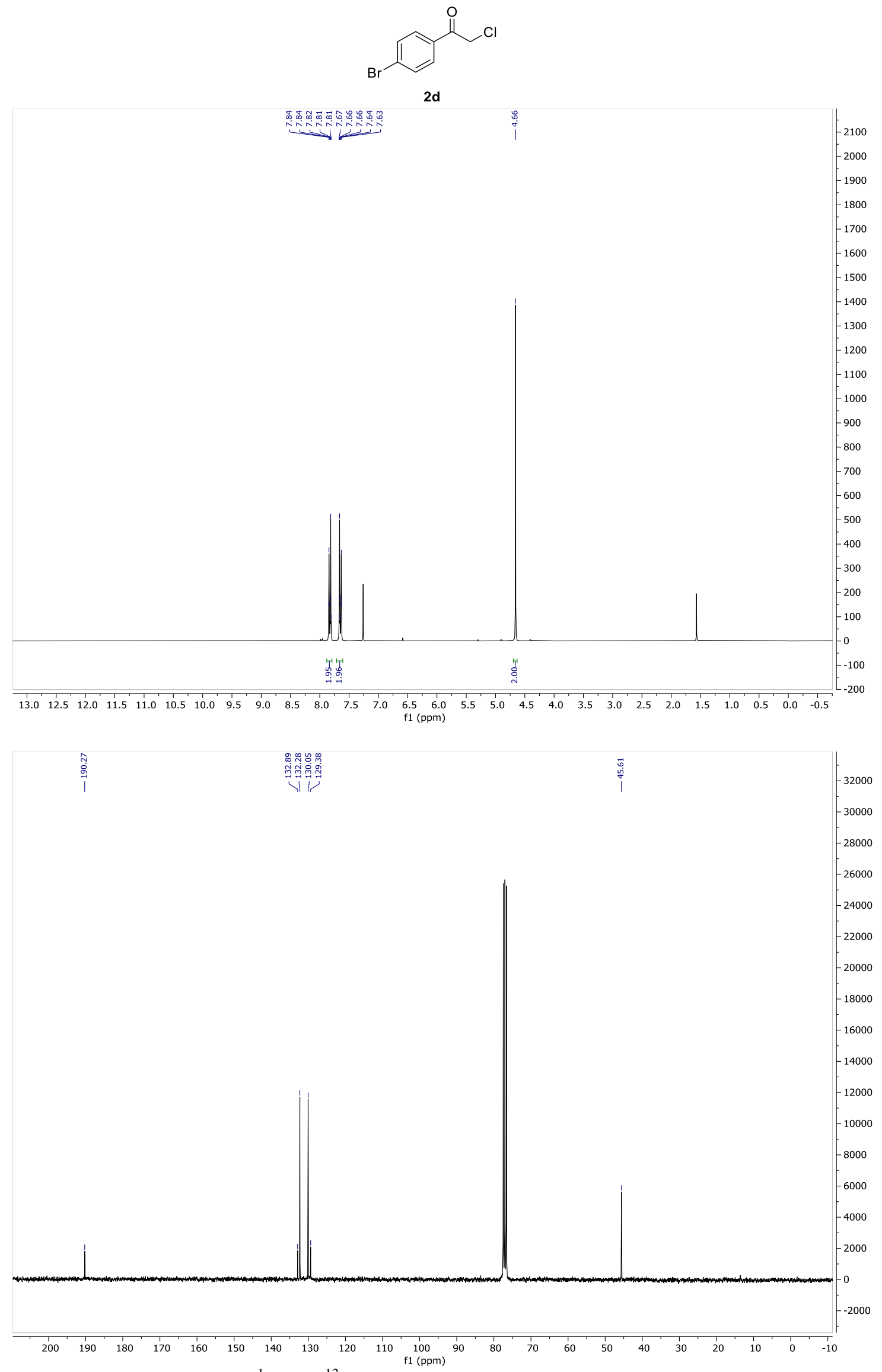

Figure S36. ${ }^{1} \mathrm{H}$ and ${ }^{13} \mathrm{C}$ NMR spectra $\left(\mathrm{CDCl}_{3}\right)$ of compound $\mathbf{2 d}$. 


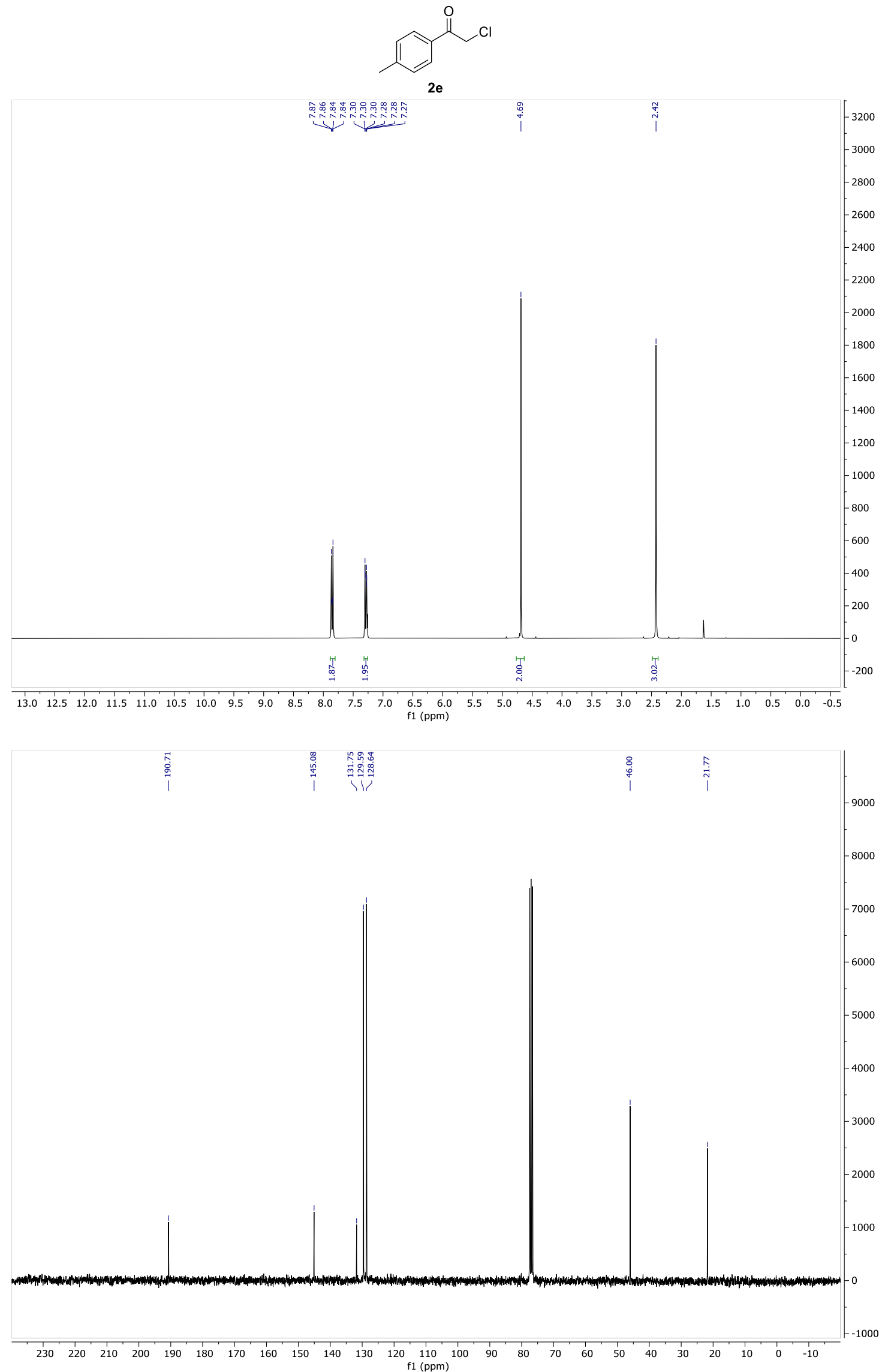

Figure S37. ${ }^{1} \mathrm{H}$ and ${ }^{13} \mathrm{C}$ NMR spectra $\left(\mathrm{CDCl}_{3}\right)$ of compound $2 \mathrm{e}$. 


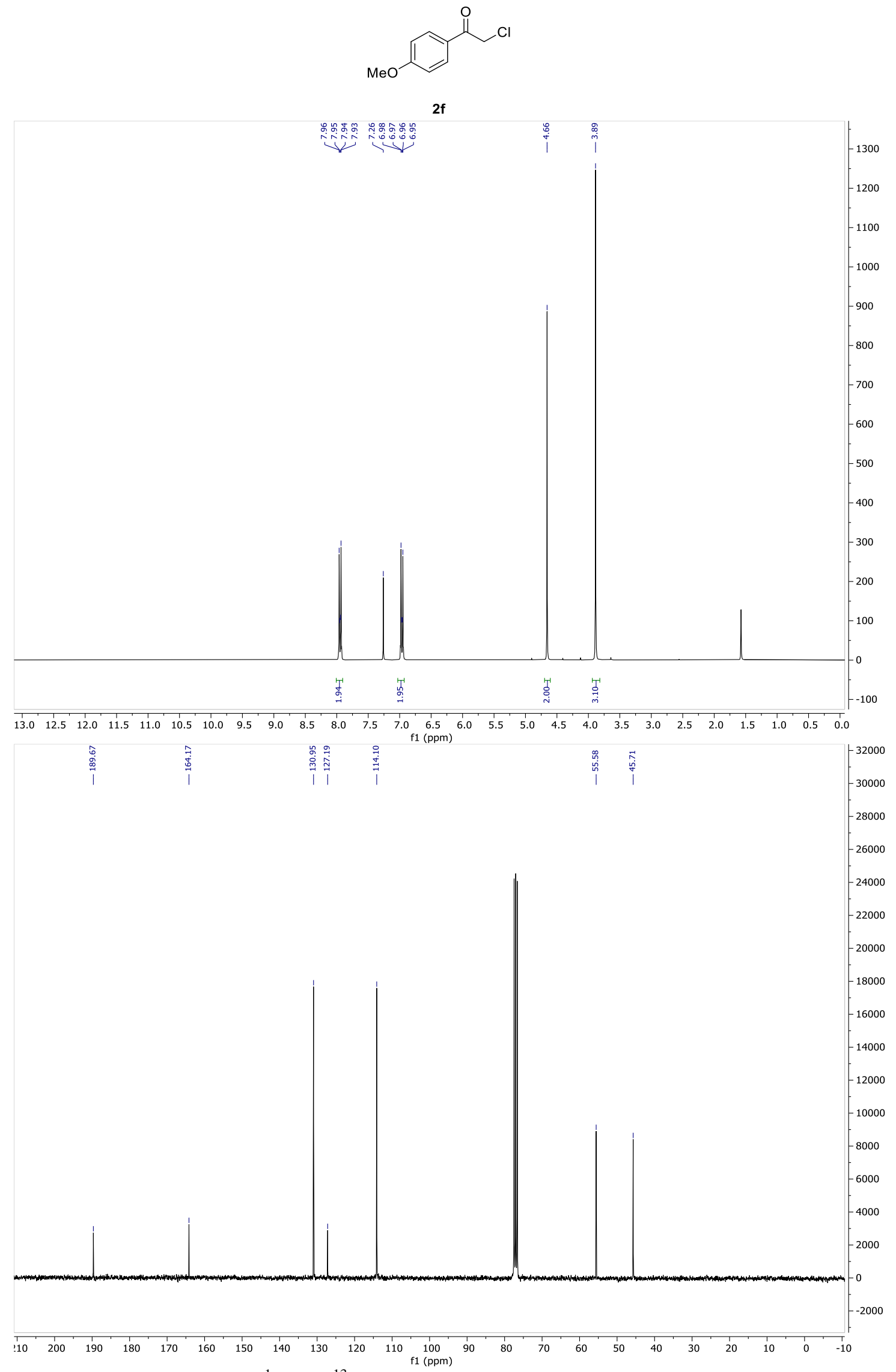

Figure S38. ${ }^{1} \mathrm{H}$ and ${ }^{13} \mathrm{C}$ NMR spectra $\left(\mathrm{CDCl}_{3}\right)$ of compound $\mathbf{2 f}$. 

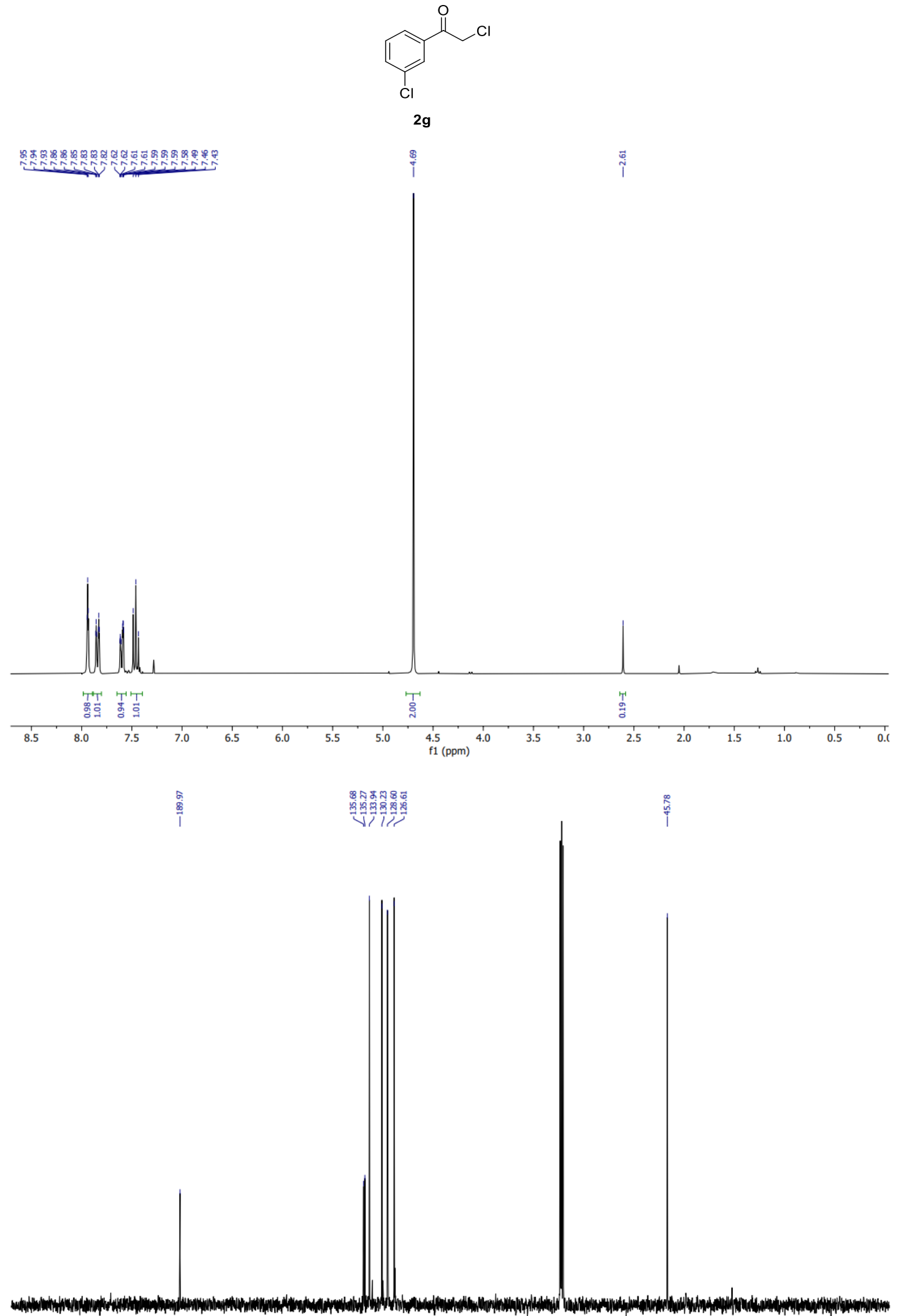

$\begin{array}{llllllllllllllllllllllllllllllll}230 & 220 & 210 & 200 & 190 & 180 & 170 & 160 & 150 & 140 & 130 & 120 & 110 & 100 & 90 & 80 & 70 & 60 & 50 & 40 & 30 & 20 & 10 & 0 & -10\end{array}$

Figure S39. ${ }^{1} \mathrm{H}$ and ${ }^{13} \mathrm{C}$ NMR spectra $\left(\mathrm{CDCl}_{3}\right)$ of compound $\mathbf{2 g}$. 




$\mathbf{2 h}$

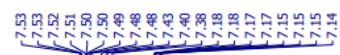

$\stackrel{\text { i }}{\text { i }}$

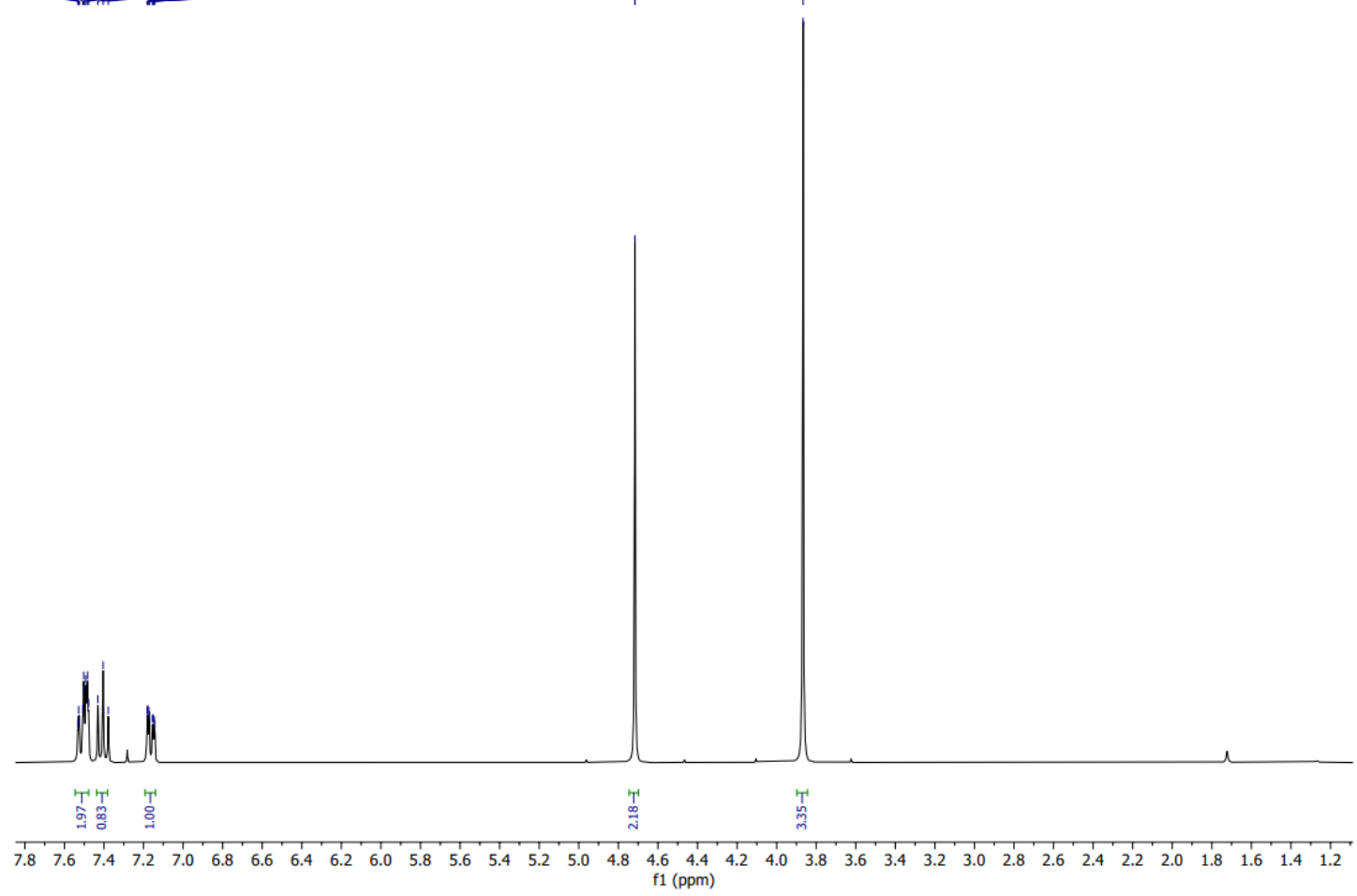

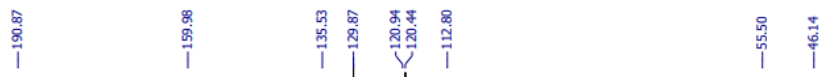

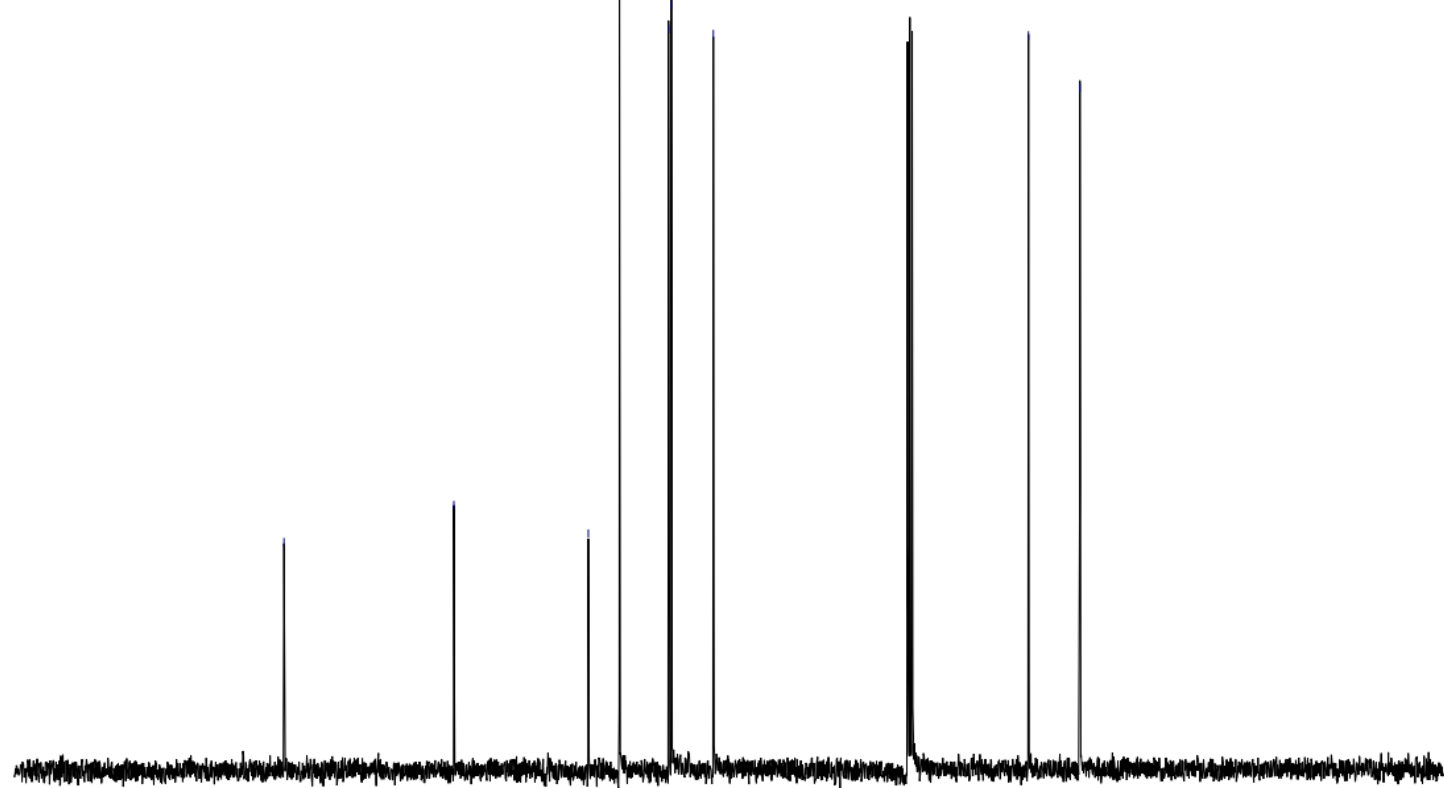

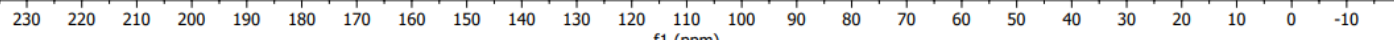

Figure S40. ${ }^{1} \mathrm{H}$ and ${ }^{13} \mathrm{C}$ NMR spectra $\left(\mathrm{CDCl}_{3}\right)$ of compound $\mathbf{2 h}$. 


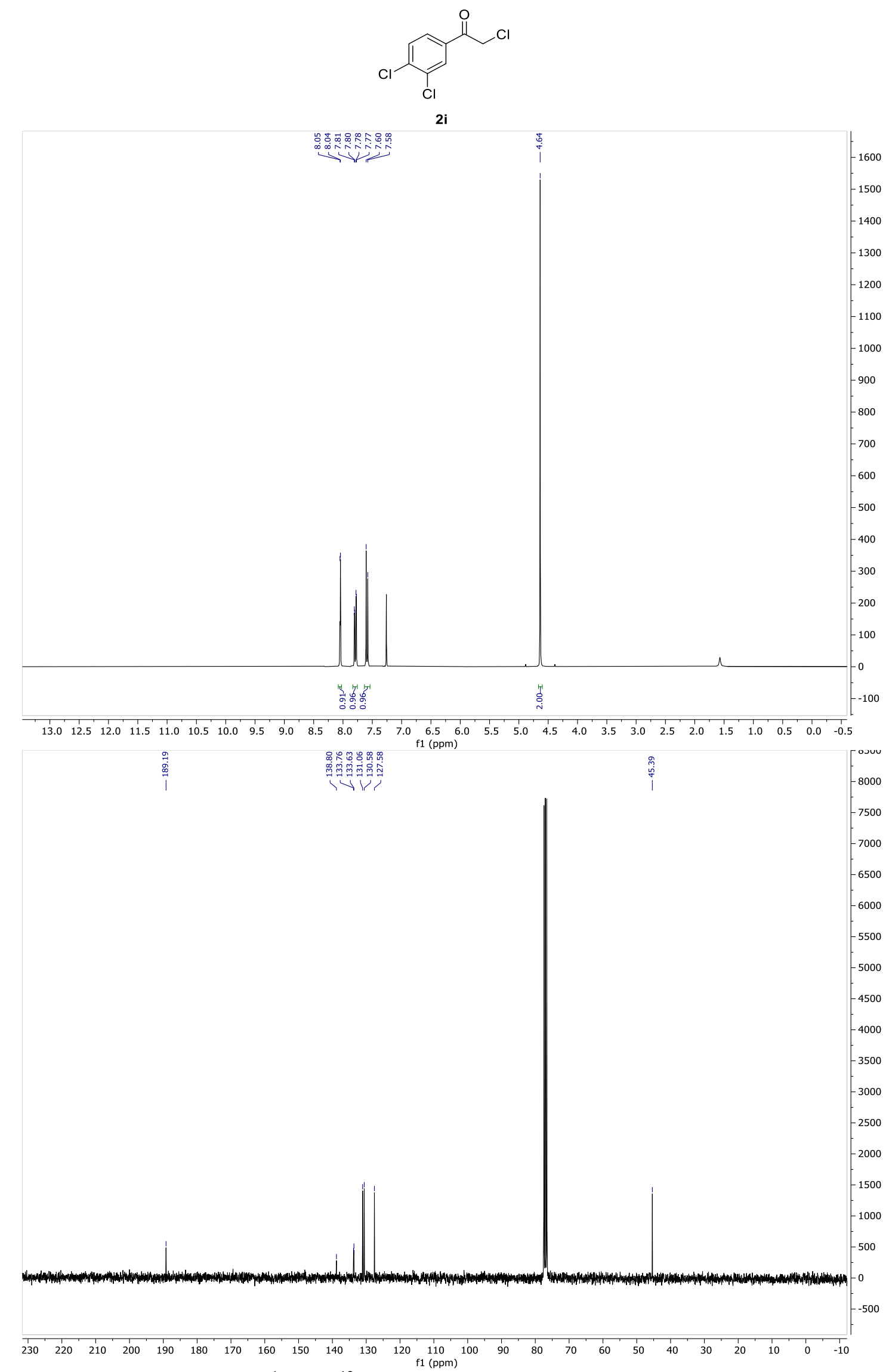

Figure S41. ${ }^{1} \mathrm{H}$ and ${ }^{13} \mathrm{C}$ NMR spectra $\left(\mathrm{CDCl}_{3}\right)$ of compound $2 \mathbf{i}$. 




Figure $\mathbf{S 4 2} .{ }^{1} \mathrm{H}$ and ${ }^{13} \mathrm{C}$ NMR spectra $\left(\mathrm{CDCl}_{3}\right)$ of compound $\mathbf{2 j}$. 


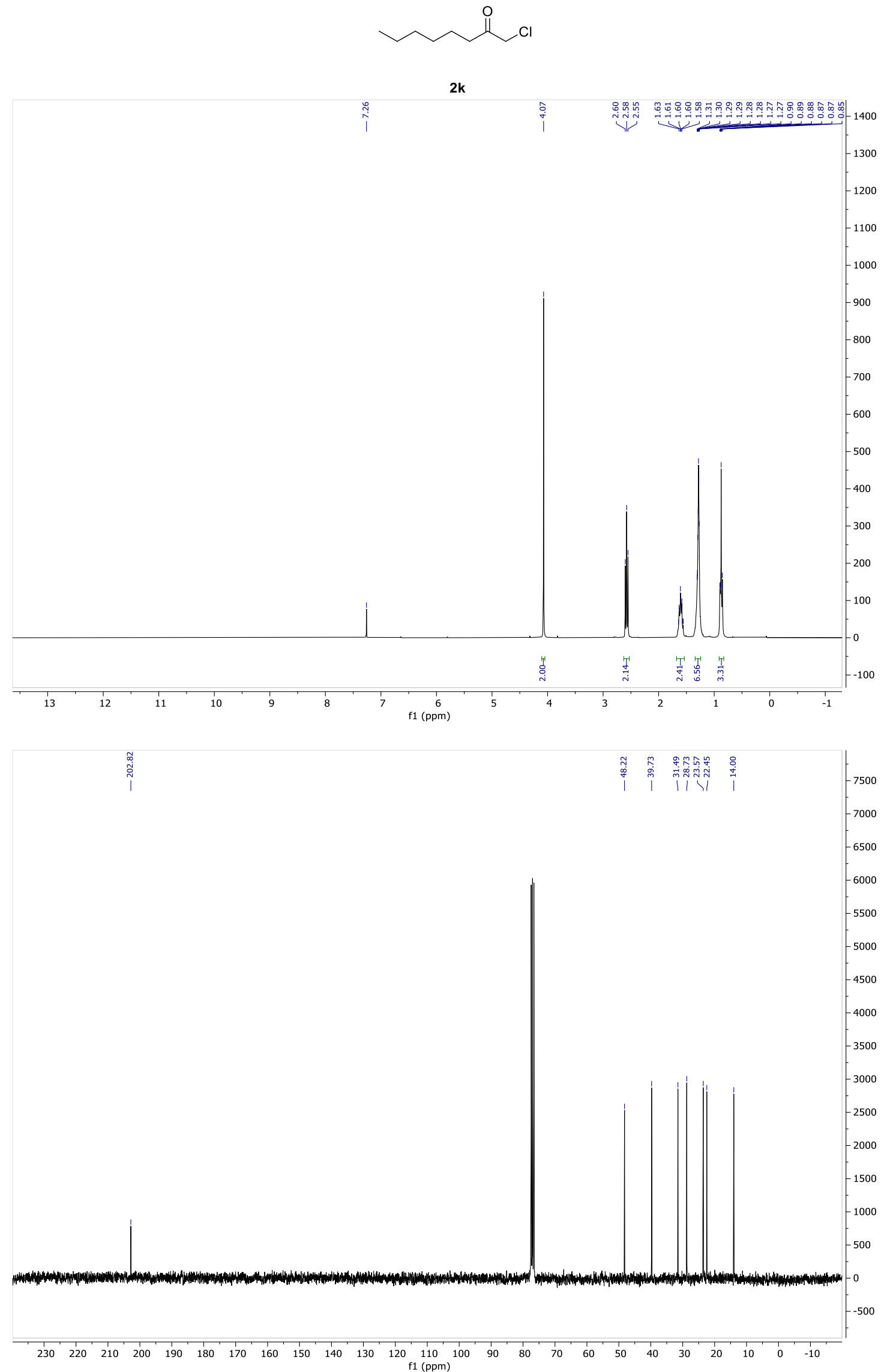

Figure $\mathbf{S 4 3} .{ }^{1} \mathrm{H}$ and ${ }^{13} \mathrm{C}$ NMR spectra $\left(\mathrm{CDCl}_{3}\right)$ of compound $\mathbf{2 k}$. 


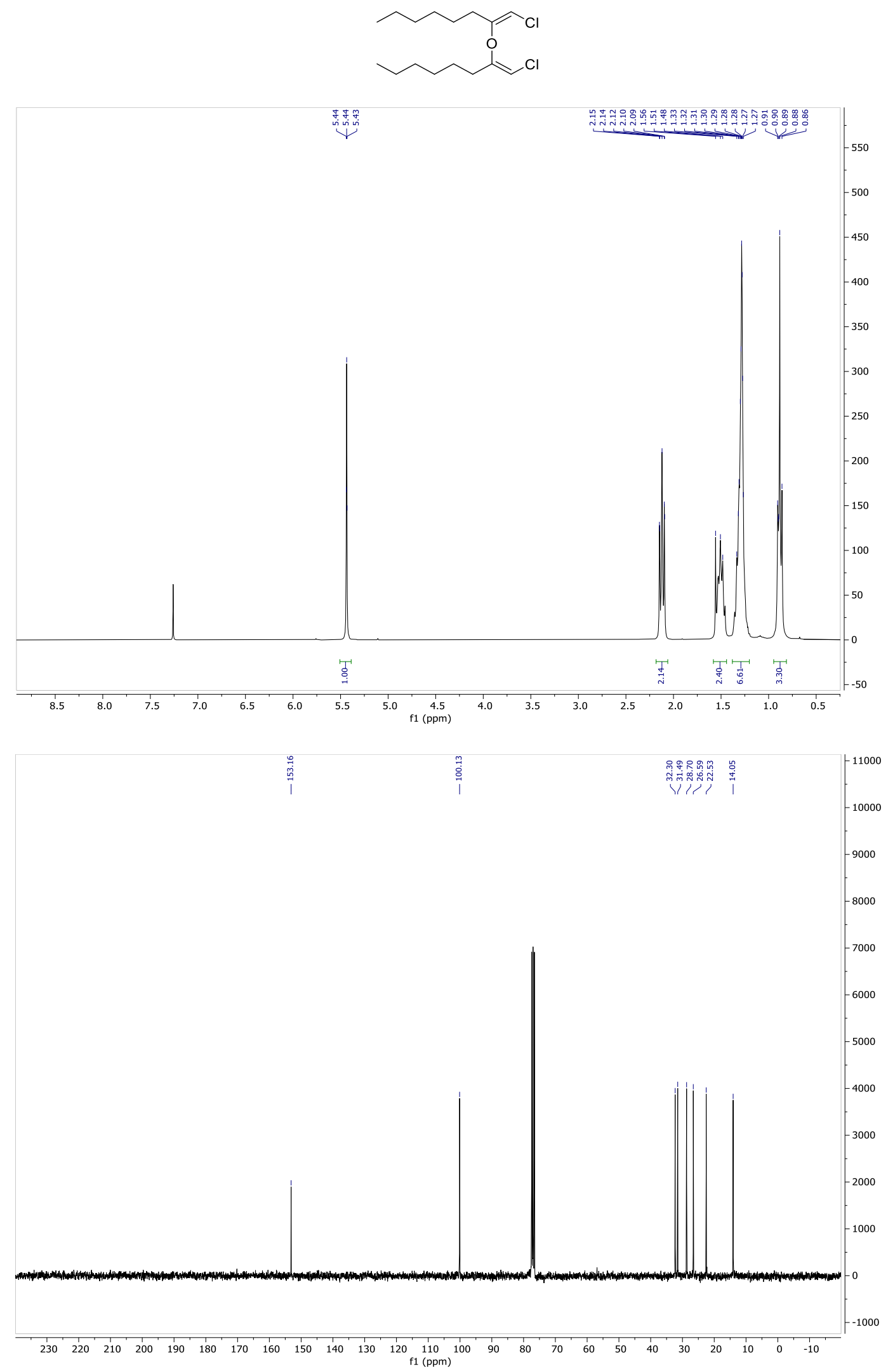






HMBC

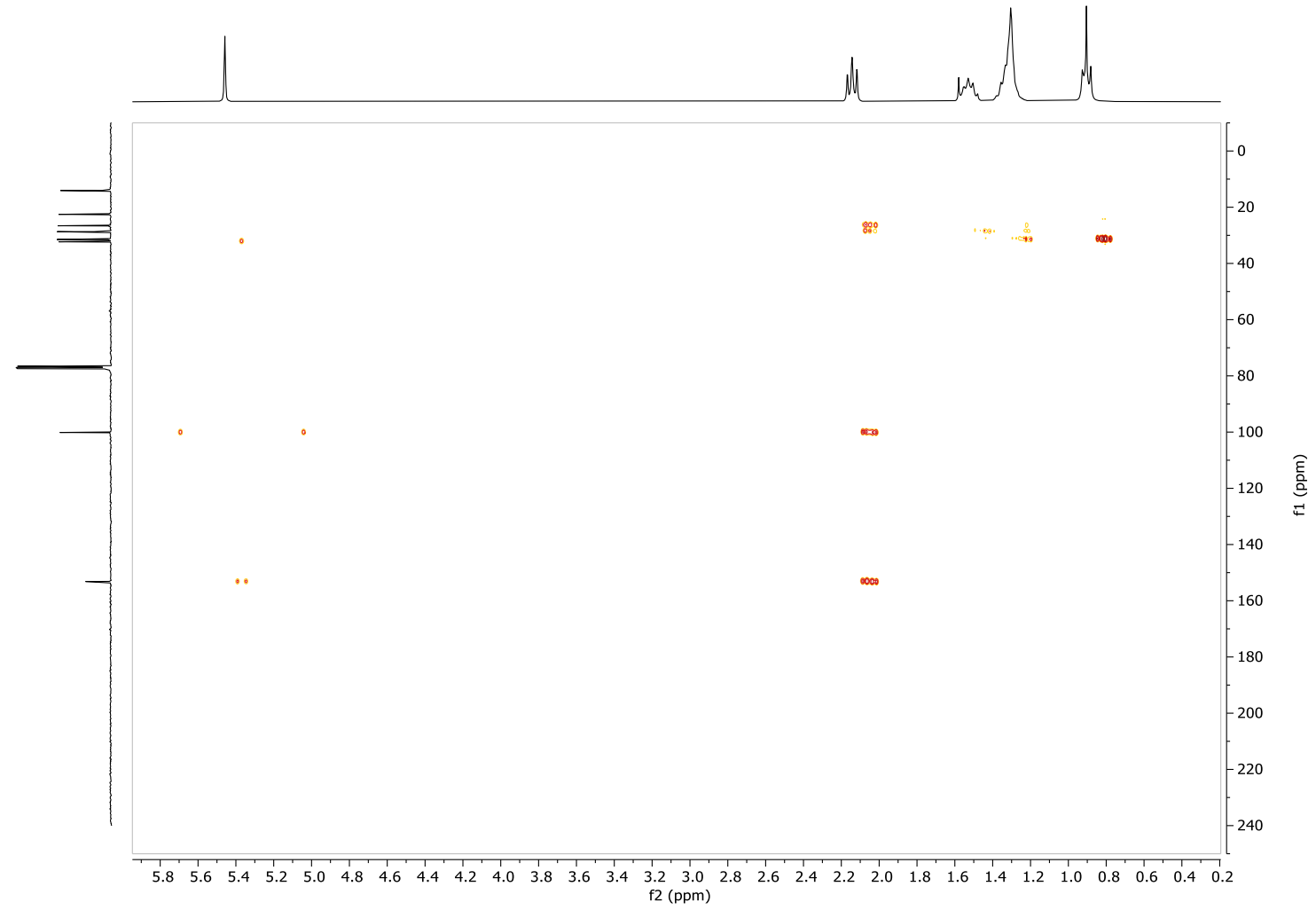


NOESY

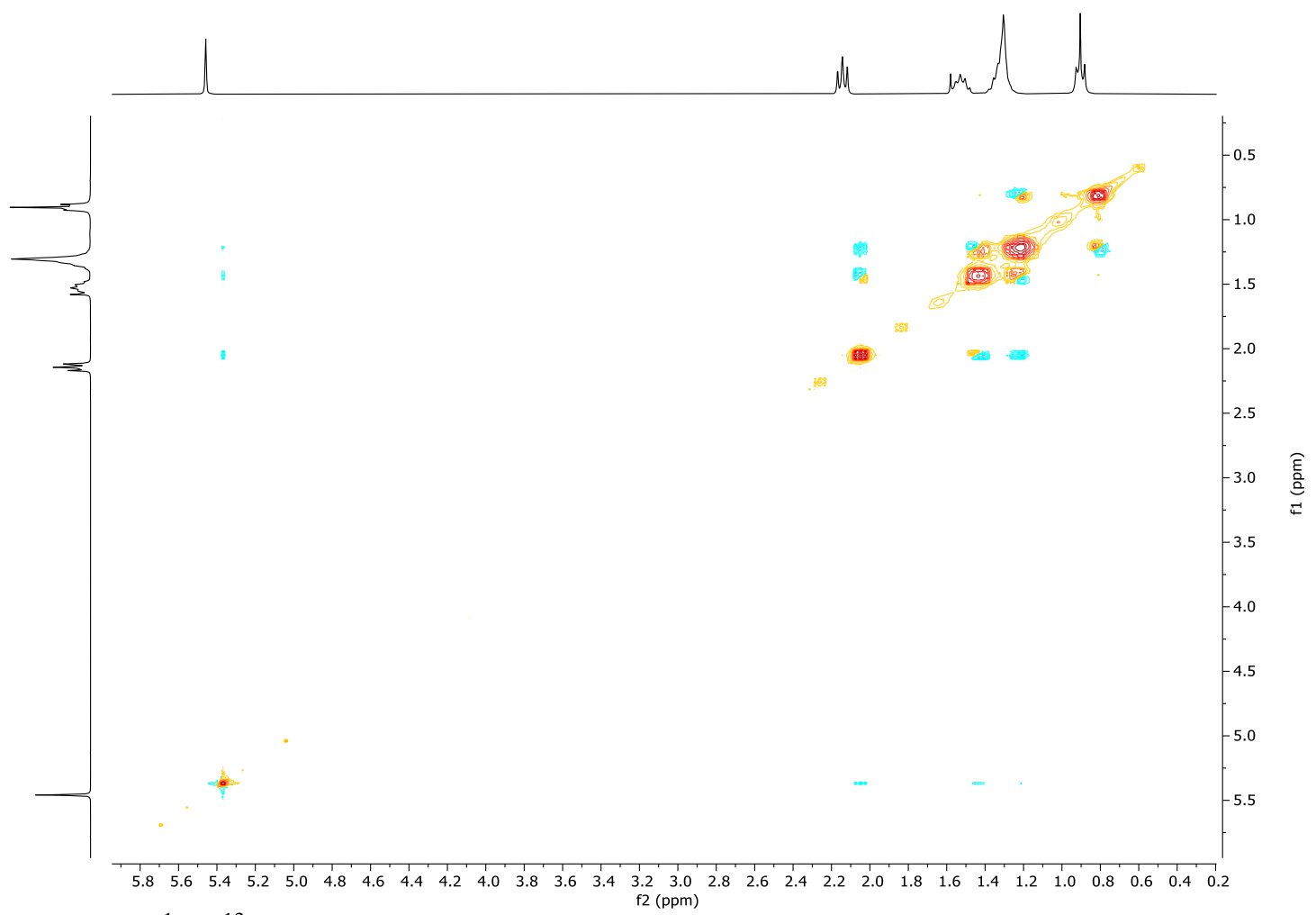

Figure S44. ${ }^{1} \mathrm{H},{ }^{13} \mathrm{C}$, DEPT, HMBC and NOESY NMR spectra $\left(\mathrm{CDCl}_{3}\right)$ of by-product detected in the hydration of $\mathbf{2 k}$. 


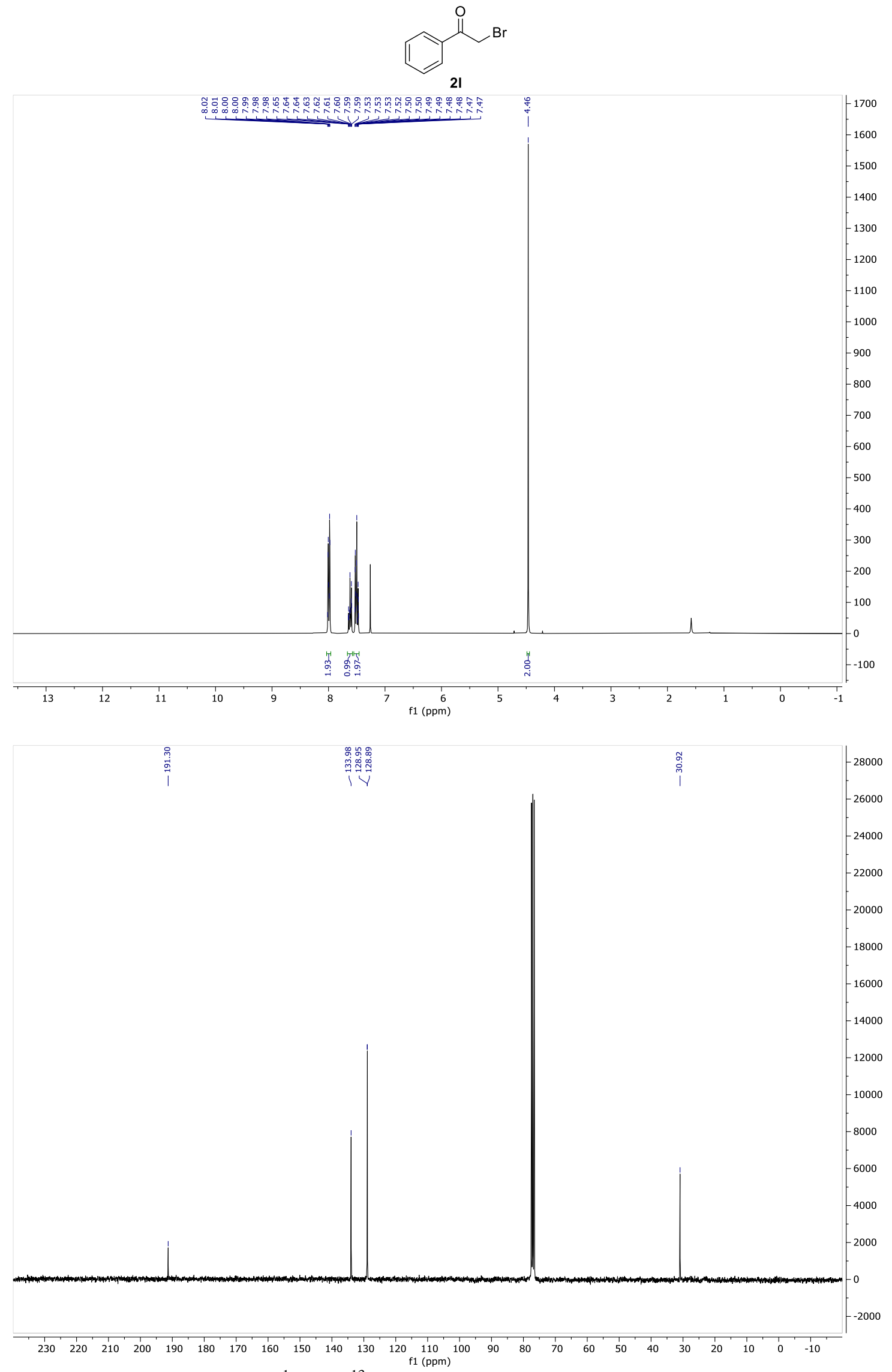

Figure S45. ${ }^{1} \mathrm{H}$ and ${ }^{13} \mathrm{C}$ NMR spectra $\left(\mathrm{CDCl}_{3}\right)$ of compound 2 I. 


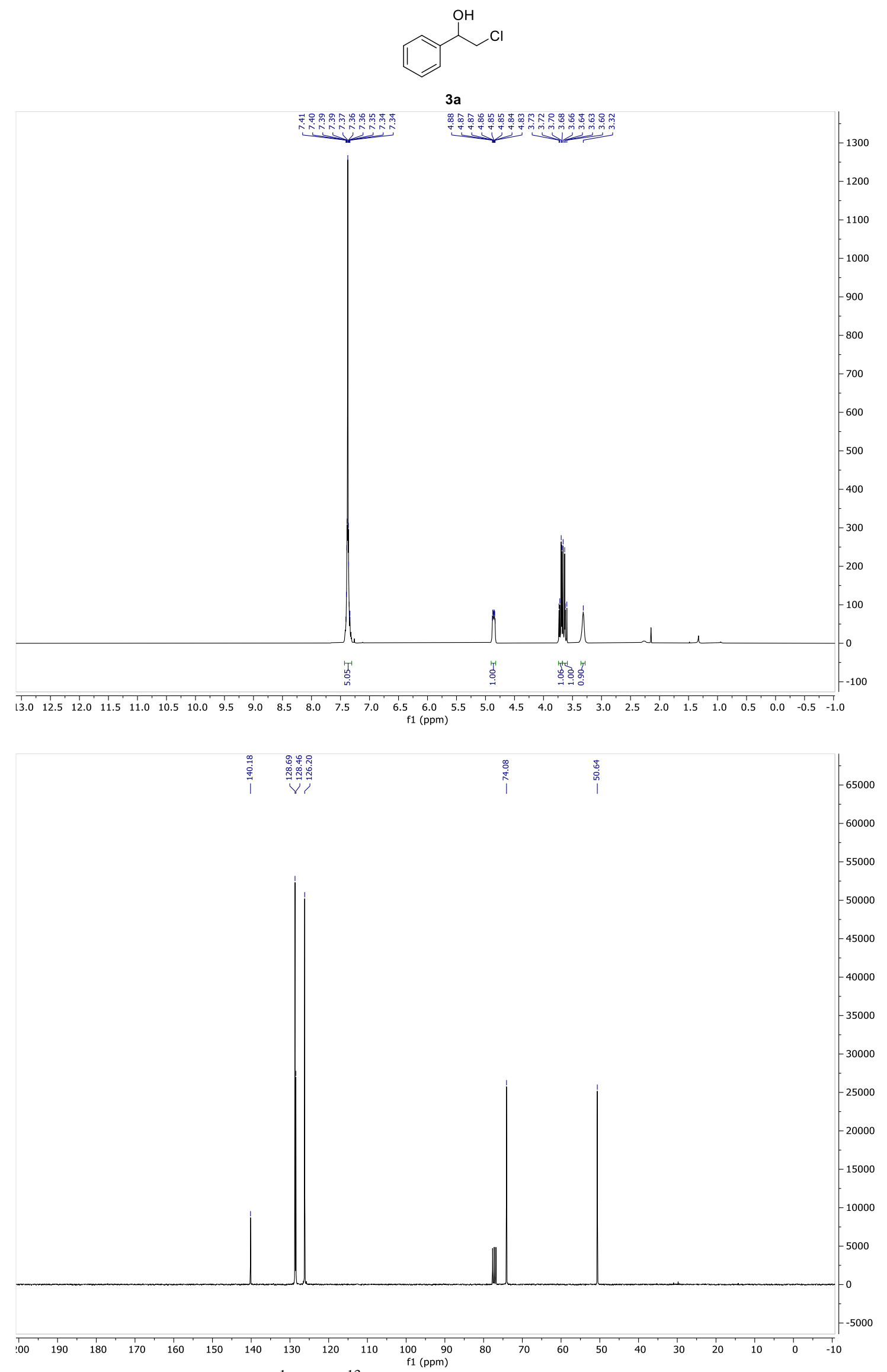

Figure S46. ${ }^{1} \mathrm{H}$ and ${ }^{13} \mathrm{C}$ NMR spectra $\left(\mathrm{CDCl}_{3}\right)$ of compound $\mathbf{3 a}$. 


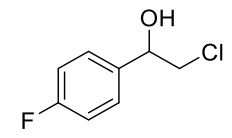

$3 b$
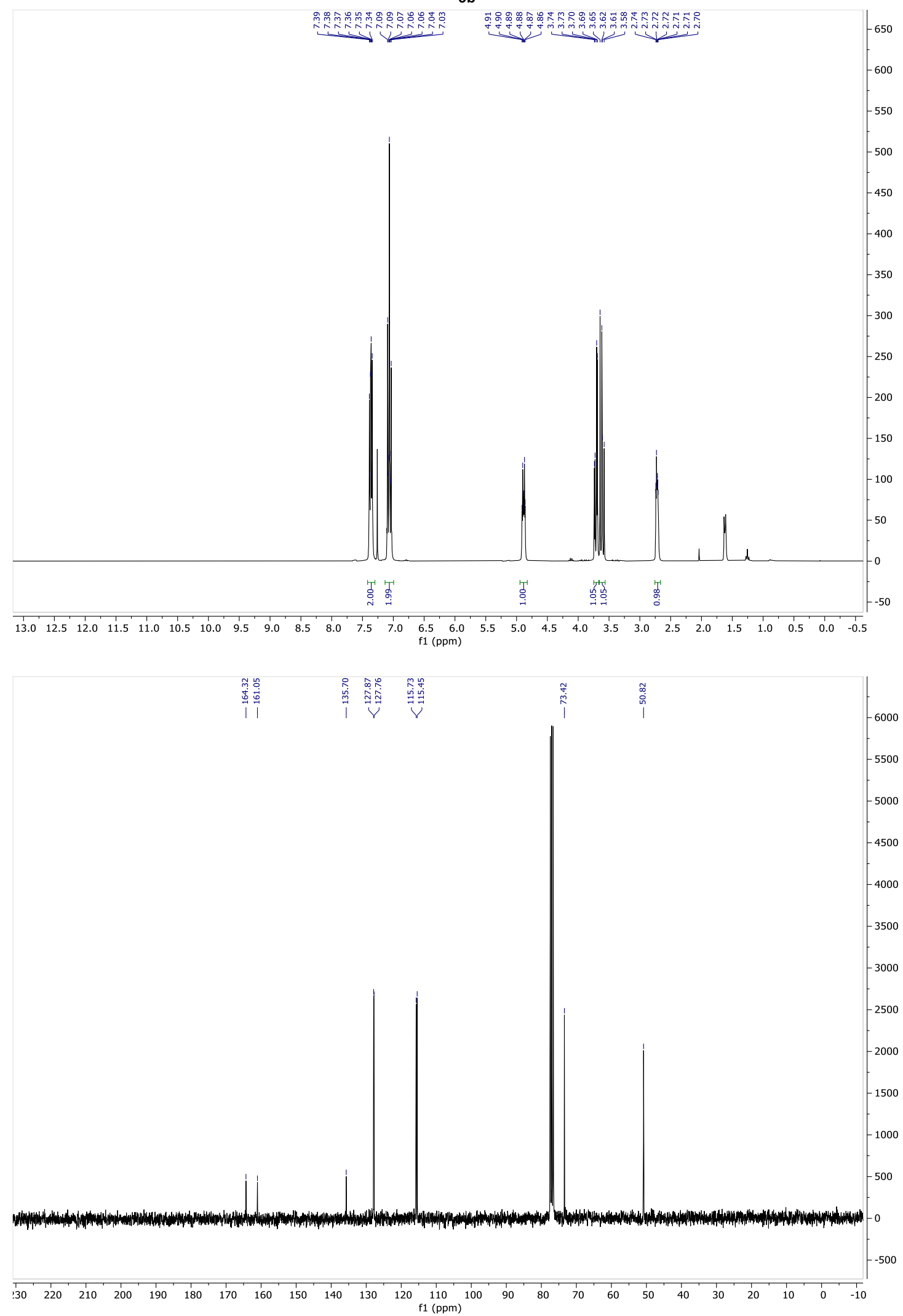


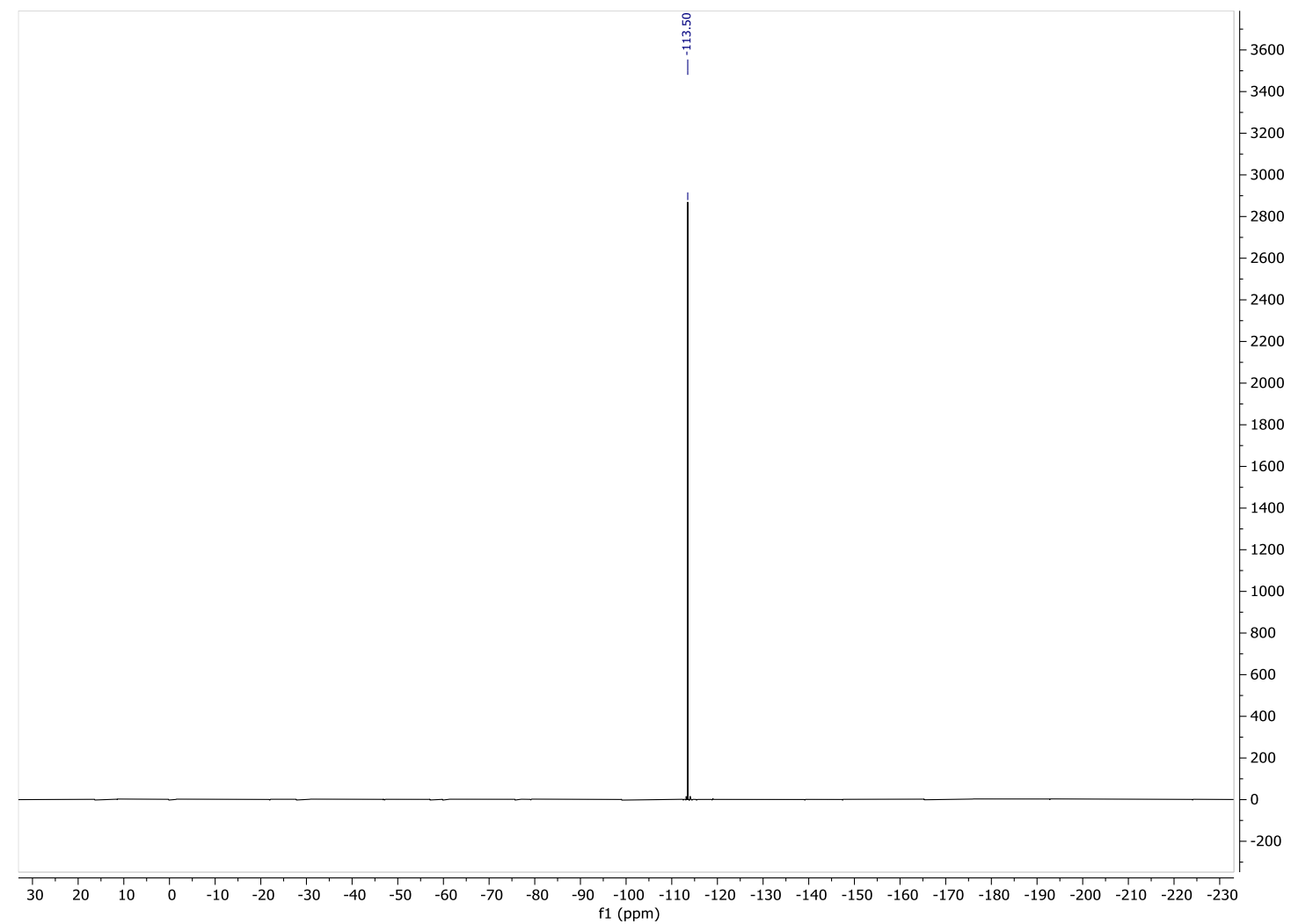

Figure S47. ${ }^{1} \mathrm{H},{ }^{13} \mathrm{C}$ and ${ }^{19} \mathrm{~F}$ NMR spectra $\left(\mathrm{CDCl}_{3}\right)$ of compound $\mathbf{3 b}$. 


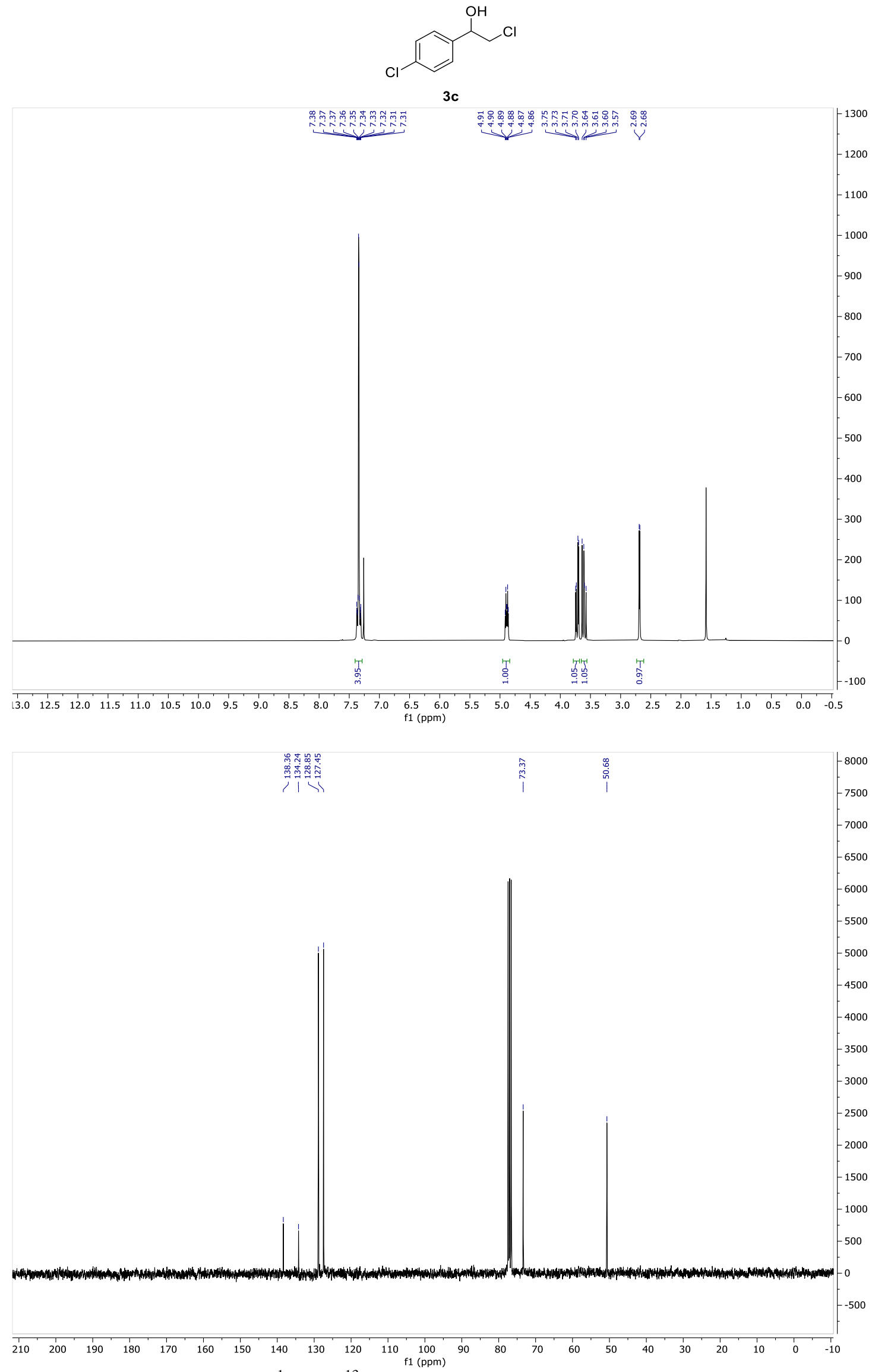

Figure $\mathbf{S 4 8} .{ }^{1} \mathrm{H}$ and ${ }^{13} \mathrm{C}$ NMR spectra $\left(\mathrm{CDCl}_{3}\right)$ of compound $\mathbf{3 c}$. 


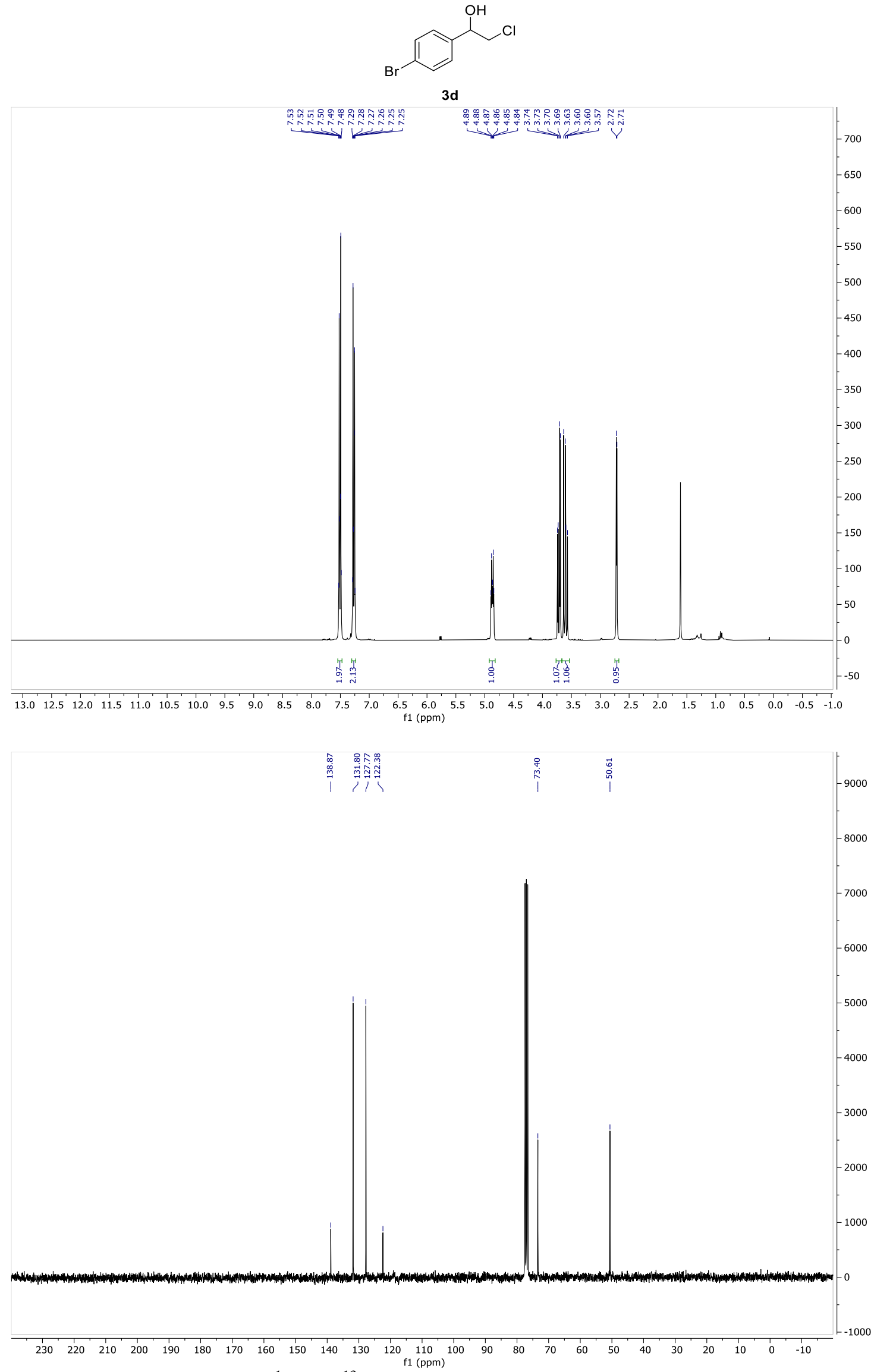

Figure $\mathbf{S 4 9} .{ }^{1} \mathrm{H}$ and ${ }^{13} \mathrm{C}$ NMR spectra $\left(\mathrm{CDCl}_{3}\right)$ of compound $\mathbf{3 d}$. 

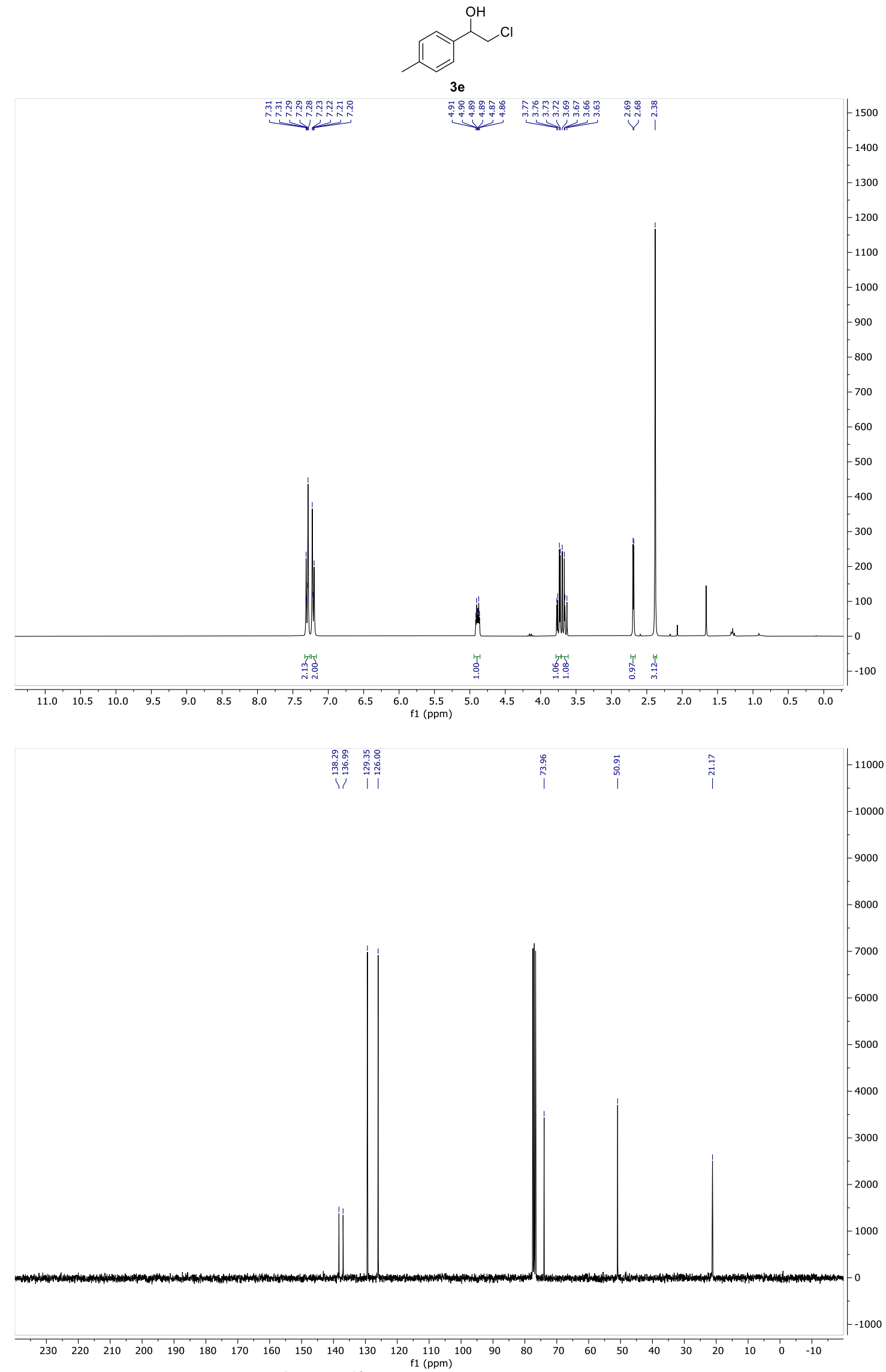

Figure S50. ${ }^{1} \mathrm{H}$ and ${ }^{13} \mathrm{C}$ NMR spectra $\left(\mathrm{CDCl}_{3}\right)$ of compound $3 \mathbf{e}$. 


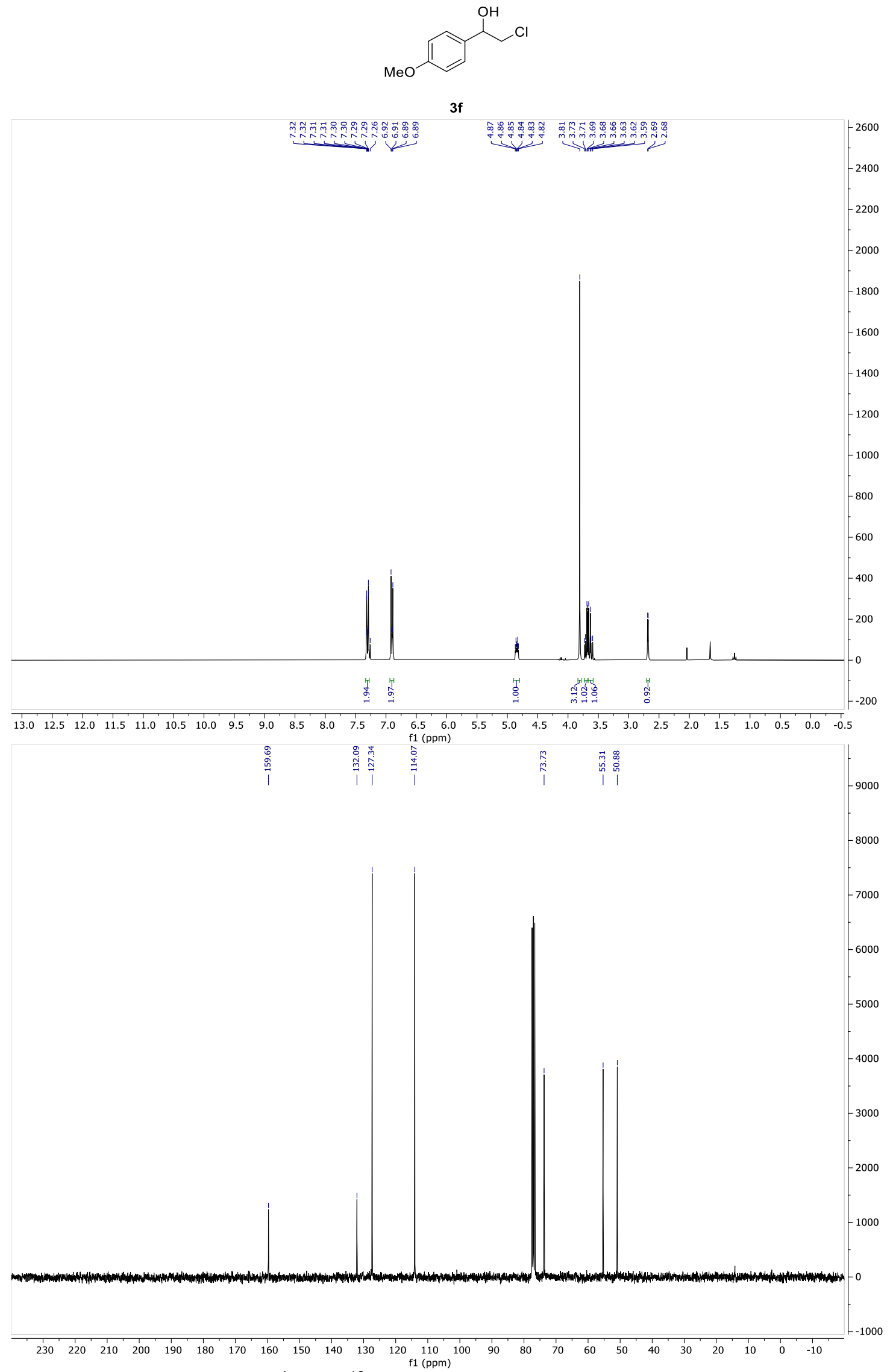

Figure S51. ${ }^{1} \mathrm{H}$ and ${ }^{13} \mathrm{C}$ NMR spectra $\left(\mathrm{CDCl}_{3}\right)$ of compound $\mathbf{3 f}$. 



Figure S52. ${ }^{1} \mathrm{H}$ and ${ }^{13} \mathrm{C}$ NMR spectra $\left(\mathrm{CDCl}_{3}\right)$ of compound $\mathbf{3 g}$. 


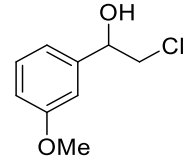

3h

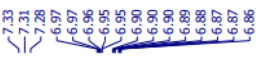

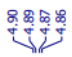

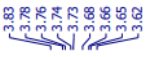
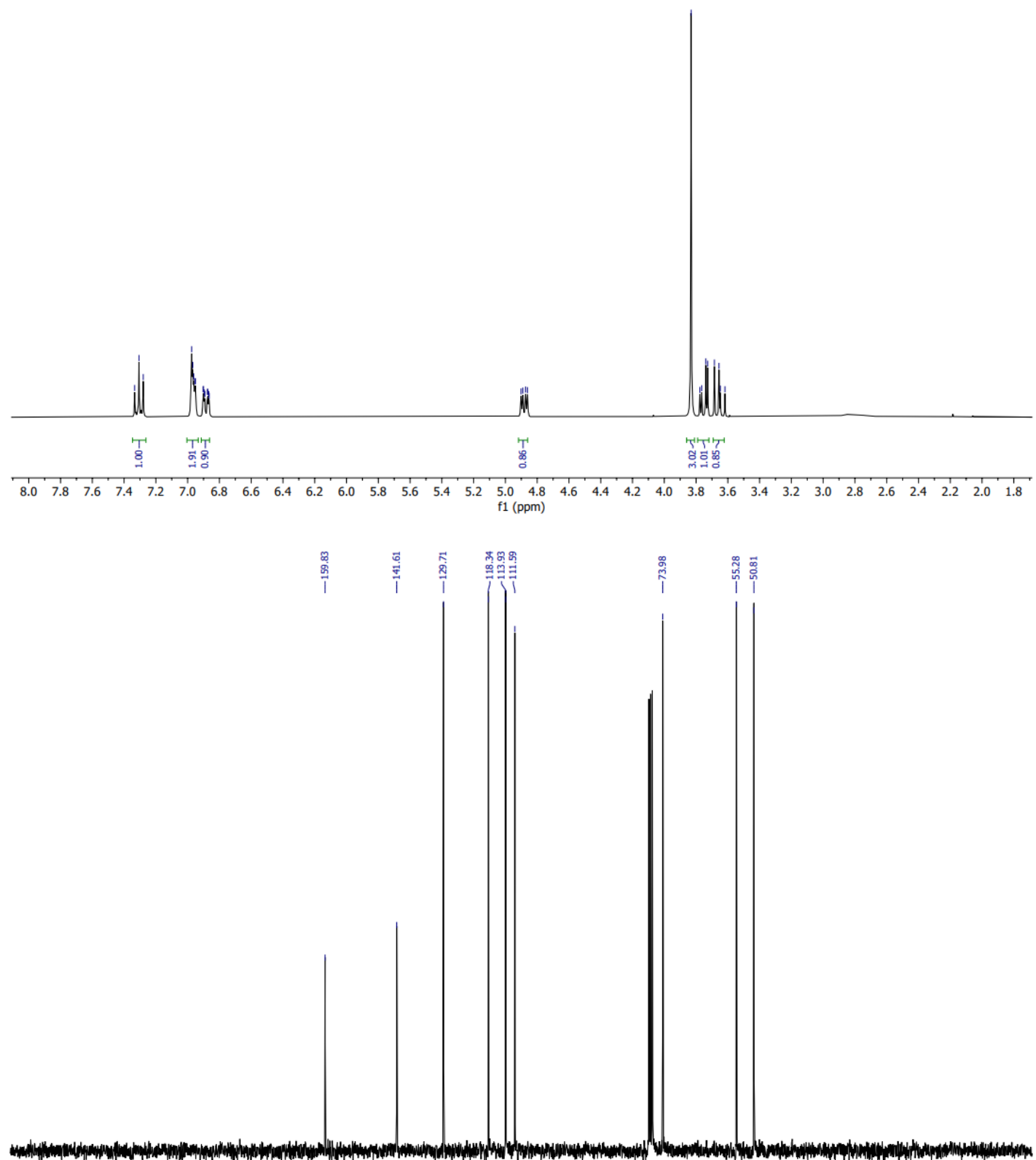

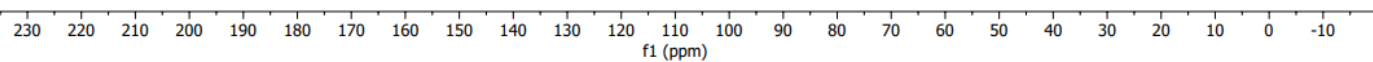

Figure S53. ${ }^{1} \mathrm{H}$ and ${ }^{13} \mathrm{C}$ NMR spectra $\left(\mathrm{CDCl}_{3}\right)$ of compound $\mathbf{3 h}$. 


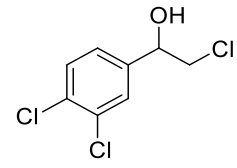

$3 \mathbf{i}$

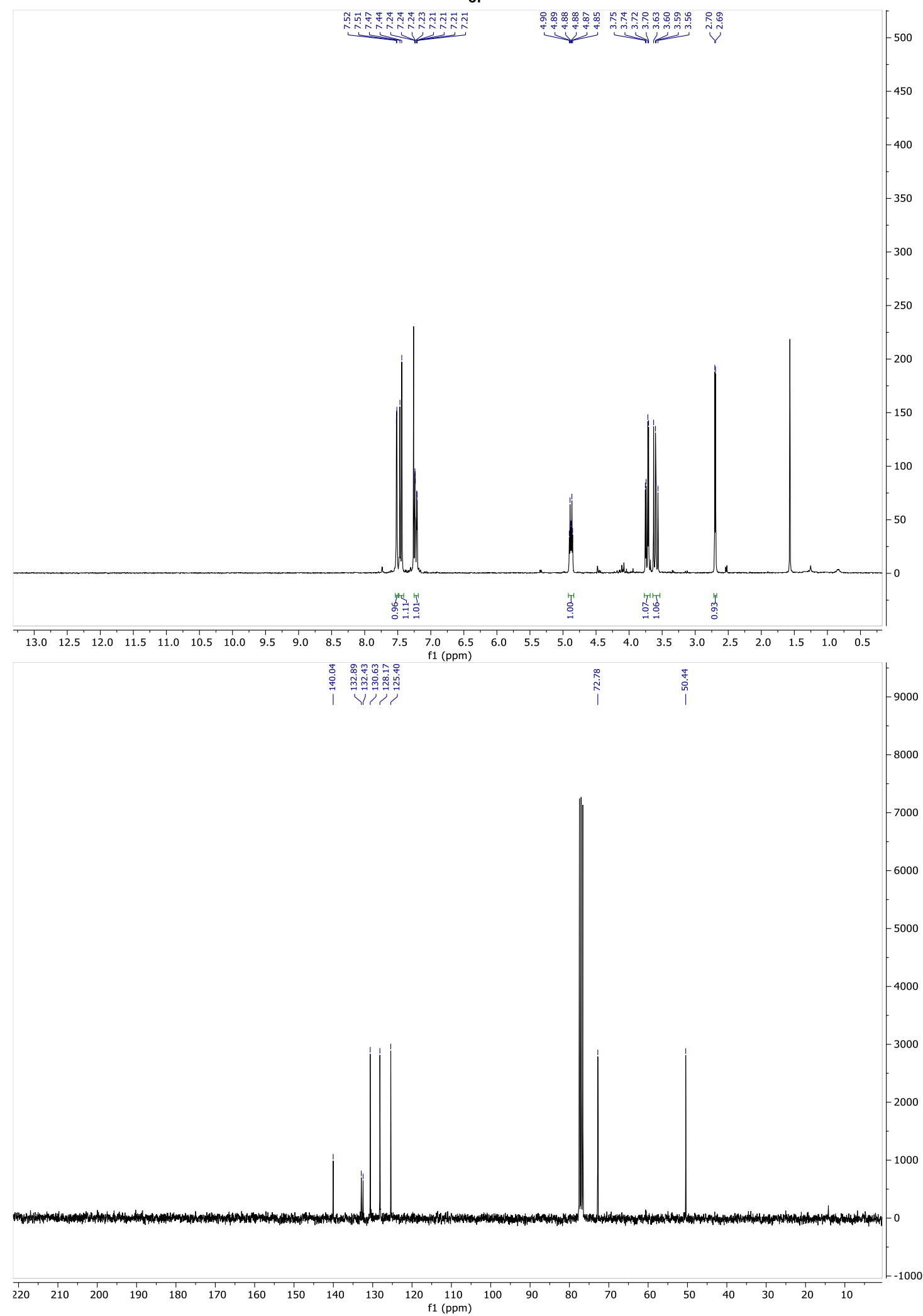

Figure S54. ${ }^{1} \mathrm{H}$ and ${ }^{13} \mathrm{C}$ NMR spectra $\left(\mathrm{CDCl}_{3}\right)$ of compound $3 \mathbf{i}$. 


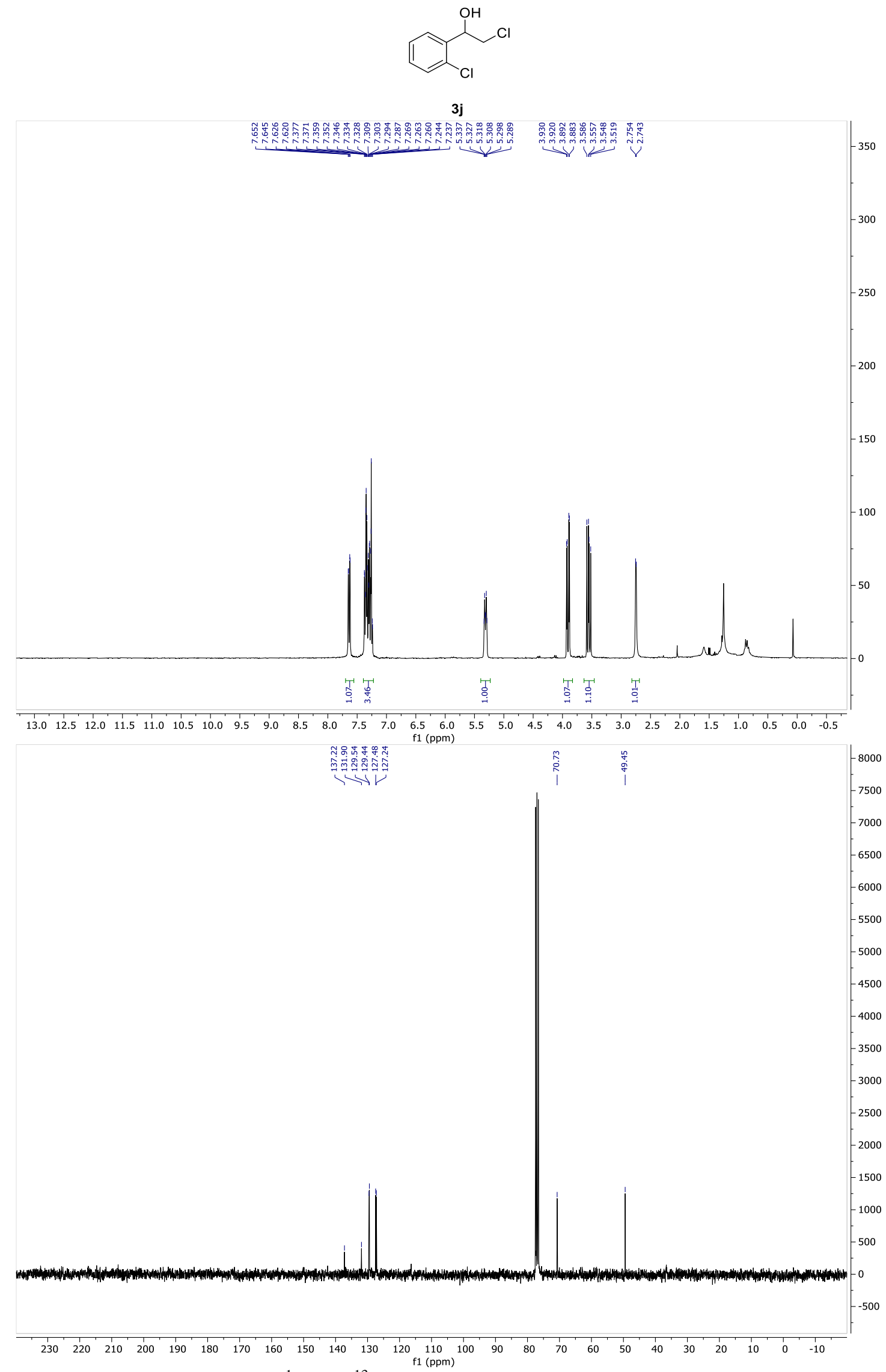

Figure S55. ${ }^{1} \mathrm{H}$ and ${ }^{13} \mathrm{C}$ NMR spectra $\left(\mathrm{CDCl}_{3}\right)$ of compound $\mathbf{3 j}$. 

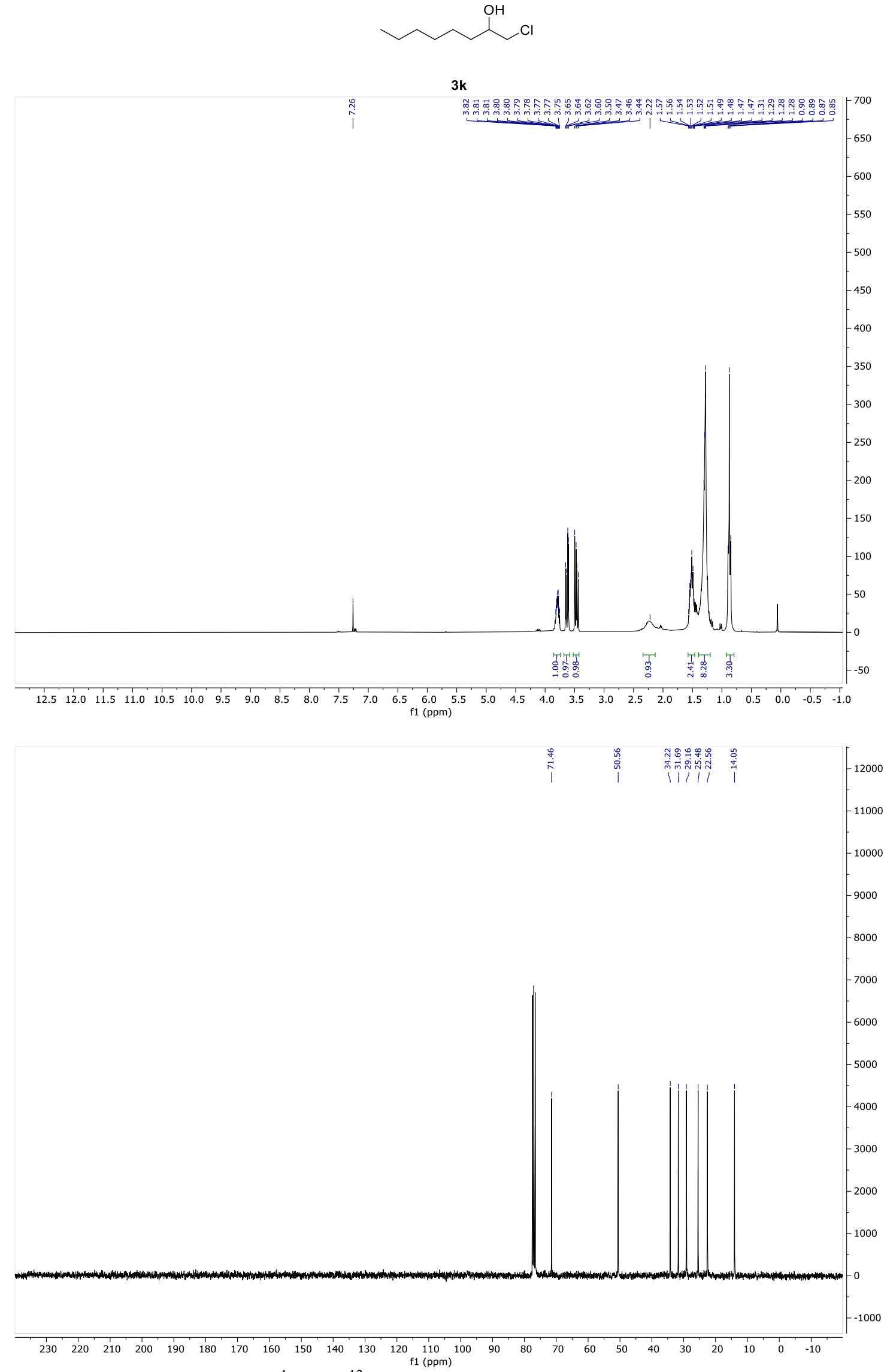

Figure S56. ${ }^{1} \mathrm{H}$ and ${ }^{13} \mathrm{C}$ NMR spectra $\left(\mathrm{CDCl}_{3}\right)$ of compound 3k. 


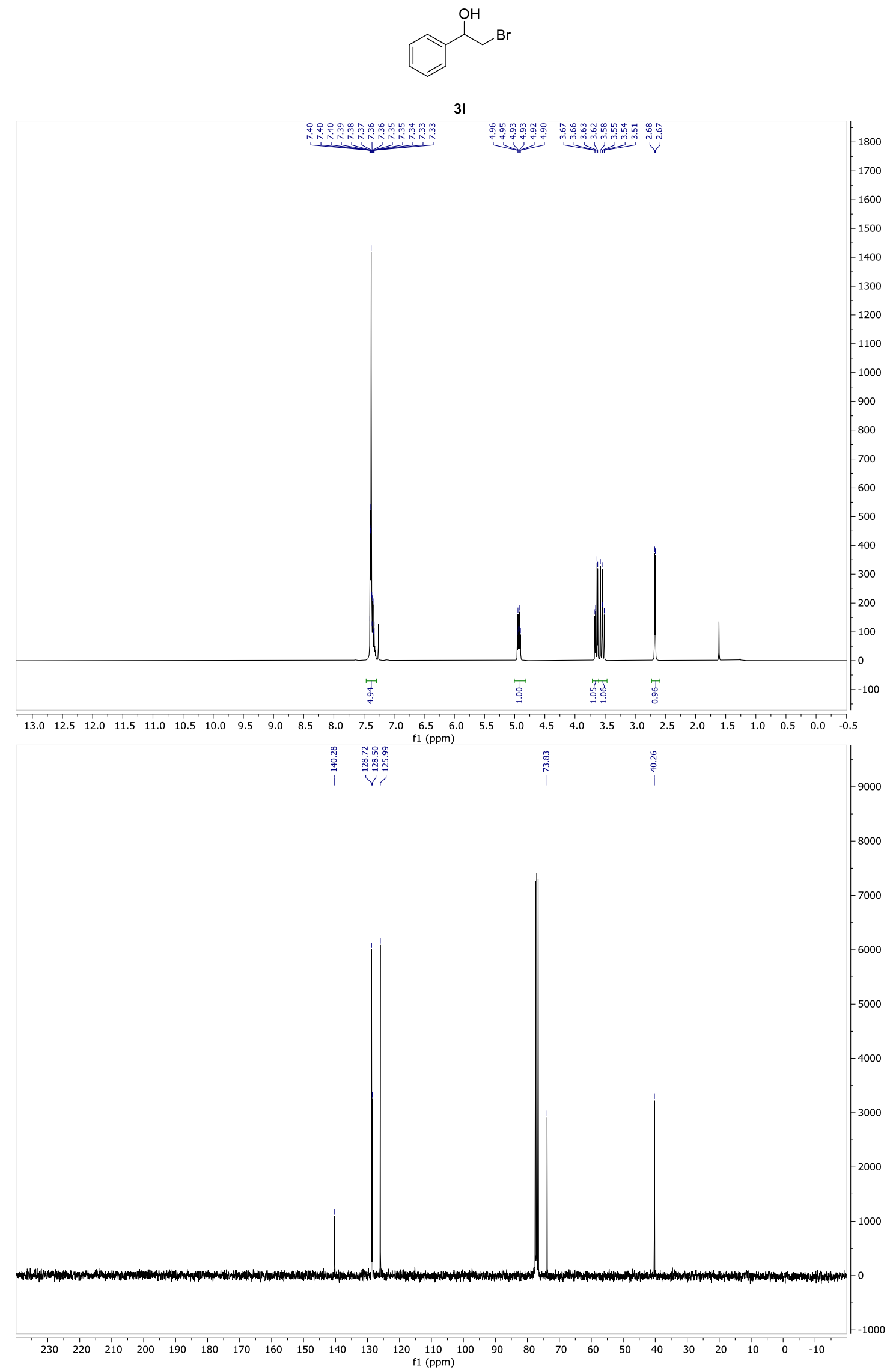

Figure S57. ${ }^{1} \mathrm{H}$ and ${ }^{13} \mathrm{C}$ NMR spectra $\left(\mathrm{CDCl}_{3}\right)$ of compound $3 \mathbf{3}$. 


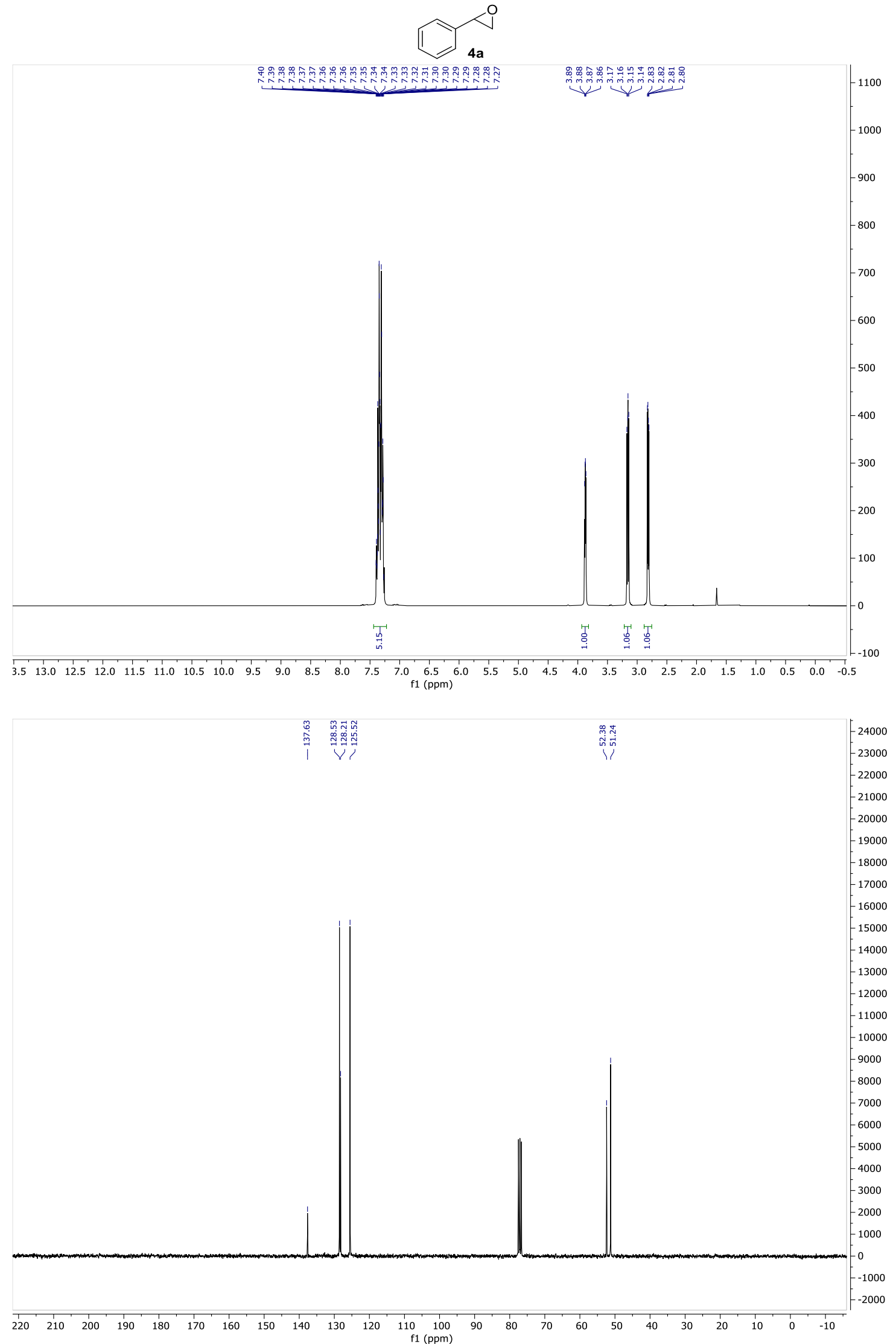

Figure S58. ${ }^{1} \mathrm{H}$ and ${ }^{13} \mathrm{C}$ NMR spectra $\left(\mathrm{CDCl}_{3}\right)$ of compound 4 a. 